\title{
Panic, Irrationality, and Herding: Three Ambiguous Terms in Crowd Dynamics Research
}

\author{
Milad Haghani $\mathbb{D}^{1},{ }^{1}$ Emiliano Cristiani, ${ }^{2}$ Nikolai W. F. Bode, \\ Maik Boltes, ${ }^{4}$ and Alessandro Corbetta ${ }^{5}$ \\ ${ }^{1}$ Institute of Transport and Logistics Studies, The University of Sydney Business School, The University of Sydney, Australia \\ ${ }^{2}$ Istituto per le Applicazioni del Calcolo "M. Picone", National Research Council of Italy, Rome, Italy \\ ${ }^{3}$ Department of Engineering Mathematics, University of Bristol, Bristol, UK \\ ${ }^{4}$ Forschungszentrum Jülich, Jülich, Germany \\ ${ }^{5}$ Eindhoven University of Technology, Eindhoven, Netherlands
}

Correspondence should be addressed to Milad Haghani; milad.haghani@sydney.edu.au

Received 4 April 2019; Revised 7 June 2019; Accepted 1 July 2019; Published 8 August 2019

Academic Editor: David F. Llorca

Copyright (c) 2019 Milad Haghani et al. This is an open access article distributed under the Creative Commons Attribution License, which permits unrestricted use, distribution, and reproduction in any medium, provided the original work is properly cited.

Background. The three terms "panic", "irrationality", and "herding" are ubiquitous in the crowd dynamics literature and have a strong influence on both modelling and management practices. The terms are also commonly shared between the scientific and nonscientific domains. The pervasiveness of the use of these terms is to the point where their underlying assumptions have often been treated as common knowledge by both experts and lay persons. Yet, at the same time, the literature on crowd dynamics presents ample debate, contradiction, and inconsistency on these topics. Method. This review is the first to systematically revisit these three terms in a unified study to highlight the scope of this debate. We extracted from peer-reviewed journal articles direct quotes that offer a definition, conceptualisation, or supporting/contradicting evidence on these terms and/or their underlying theories. To further examine the suitability of the term herding, a secondary and more detailed analysis is also conducted on studies that have specifically investigated this phenomenon in empirical settings. Results. The review shows that (i) there is no consensus on the definition for the terms panic and irrationality and that (ii) the literature is highly divided along discipline lines on how accurate these theories/terminologies are for describing human escape behaviour. The review reveals a complete division and disconnection between studies published by social scientists and those from the physical science domain and also between studies whose main focus is on numerical simulation versus those with empirical focus. (iii) Despite the ambiguity of the definitions and the missing consensus in the literature, these terms are still increasingly and persistently mentioned in crowd evacuation studies. (iv) Different to panic and irrationality, there is relative consistency in definitions of the term herding, with the term usually being associated with '(blind) imitation'. However, based on the findings of empirical studies, we argue why, despite the relative consistency in meaning, (v) the term herding itself lacks adequate nuance and accuracy for describing the role of 'social influence' in escape behaviour. Our conclusions also emphasise the importance of distinguishing between the social influence on various aspects of evacuation behaviour and avoiding generalisation across various behavioural layers. Conclusions. We argue that the use of these three terms in the scientific literature does not contribute constructively to extending the knowledge or to improving the modelling capabilities in the field of crowd dynamics. This is largely due to the ambiguity of these terms, the overly simplistic nature of their assumptions, or the fact that the theories they represent are not readily verifiable. Recommendations. We suggest that it would be beneficial for advancing this research field that the phenomena related to these three terms are clearly defined by more tangible and quantifiable terms and be formulated as verifiable hypotheses, so they can be operationalized for empirical testing.

\section{Introduction}

As researchers working in the field of pedestrian dynamics, we have experienced that a presentation of a piece of research on the topic of crowd evacuation, whether to an academic audience or lay audience, barely goes by without researchers being confronted with these questions: How about the effect of panic? How do you model/experiment panic? To a lesser 
extent, we also similarly receive questions of this nature during peer review processes. The question is also often accompanied by follow-up questions on irrational behaviour during evacuations and herding phenomena and how we take those into account in our computational models or experimentations.

We have also observed that these debates are often not resolved with a rigorous argument based on facts and empirical evidence and are, rather, addressed with some level of speculation and resorting to intuition. Nevertheless, researchers often concede that these might be limitations of their study and phenomena that they still have not been able to tackle. Sometimes, researchers take a more defensive position facing this question and present counterarguments that are meant to dismiss these phenomena as matters that should not concern us when designing our research experiments or formulating our models.

The question that arises is why, after so many years of research in this field, have these terms remained intractable? Does this stem from a lack of clear definitions and/or a lack of well-conditioned theoretical conceptualisation? Is this a sign that these terms are still not well defined and that they may, to some degree, be misdirecting the research in this field?

The issue of panic constitutes a rather frequent disclaimer at the discussion section of publications on crowd evacuation dynamics and a common ground for criticising the modelling and experimentation efforts in this field [1]. Such disclaimers often appear in wordings such as: These experiments were conducted under nonpanic conditions [2], or the influence of panic has been excluded from the experiment/model $[3,4]$. This gives the indication that simulating/modelling panic is going to be a future development in this field something that the research is headed towards, but one that we have not been able to tackle just yet.

What is, however, very clear is that the terms, panic, irrationality, and herding are among the most ubiquitous terms in the crowd dynamics literature. A peculiar characteristic is that they are used as commonly shared language between the scientific literature, the public, and the media to describe collective evacuation behaviour [5]. As stated by Quarantelli [5], "what constitutes panic is illustrated by presentations of anecdotal examples from stories of disaster behavior in journalistic and popular sources". Here, we investigate what level of consensus exists on their definition and meaning. We survey the scientific literature of crowd dynamics and analyse the use of these three terms with the aim of identifying (i) whether the literature offers unified definitions, (ii) how different segments of the literature view these terms and their theories in general, (iii) how well supported they are in various segments of the literature, and (iv) how they can potentially influence experimentation, modelling, and management practices in this field.

\section{Methods}

The main purpose of the review is to perform a structured literature search on the use of the terms panic, irrationality, and herding in the context of emergency evacuation of crowds. This will help to establish whether unified definitions can be identified, and it will identify possible inconsistencies or contradictions. In performing this analysis, we also aim to provide an overall reflection of how different research fields perceive each of these terms. The literature review puts together studies from a range of disciplines including physical sciences, social sciences, and biological sciences.

The structured literature analysis is mainly performed on direct quotes from peer-reviewed research articles where these terms have appeared. The main criterion for the selection of the underlying studies was that they had to be exclusively in the context of emergency behaviour, and particularly the behaviour of humans within crowds. For example, the use of the term herding in financial or other contexts where the term is frequently used is not considered here.

Using Scopus as our primary database, we performed title-keywords-abstract searches by applying all possible combinations between the terms "pedestrian, evacuation, crowd, escape, disaster, emergency", and the set of three focus terms of this study "panic, irrational, herd" while separating them by the operator "AND". Each search outcome was limited to Articles and Reviews as Document Types, and exclusively Journals as Source Types. No particular date was specified. This search was initially performed in August 2018. It was subsequently updated in January 2019, limiting the outputs to 2018 and 2019 as Year of publication. For each search, the outputs underwent an initial screening to identify the relevant articles. This screening was performed first on the title of the articles that appeared in search outputs and then on their abstract and keywords only if necessary (i.e., only if the title did not give clear indication of whether the study would be potentially relevant to the content of the review). The search was also supplemented by a prior and less systematic search on a personal reference database that includes nearly 2000 selected articles in the context of crowd dynamics, as well as a variety of Google Scholar searches using similar combination of terms used in Scopus.

This process generated a shortlist of nearly 200 articles whose full texts were screened for the purpose of extracting quotes relevant to the context of this review. The full text of each article was searched for the use of the terms 'panic', 'irrational(ity)', and 'herd(ing)' separately. The criteria for choosing quotes where these terms appeared were that the quote has to convey some form of definition on the term, characterise the term (or its underlying phenomenon), or make some comment on the validity of their underlying theory or the commonness of the phenomenon real-life emergencies. We use these broad inclusion criteria to achieve a comprehensive and objective perspective on how these terms are perceived and used in various subdivisions of the literature.

Out of the nearly 200 shortlisted articles whose full texts were analysed for the use of these terms, half of them (101 items) produced at least one quote that met our criteria outlined above. These quotes were extracted from each article and were stored in separate Word files for further subsequent analyses. In the subsequent analyses, mainly for the purpose of keeping this review to a reasonable length, quotes within 
studies that had produced more abundant material had to be prioritised. In such cases, where a study had produced several and often lengthy quotes relevant to our review topic, the quotes with similar content were compared together and briefest ones were chosen. Also, for quotes in which more than one of the three terms had appeared, the quote was only categorised in one of the three sections related to these terms by identifying the term that was dominant in the quote (i.e., the term that constituted the primary theme of the quote). This way, we avoided repeating individual quote for the analyses on our three terms.

The selected quotes were subsequently further analysed and categorised. We differentiated between the quotes in terms of whether they offer a definition/characterisation on the term or just comment on the commonness/likeliness of the underlying phenomenon. Where possible we also recorded whether the quote sentiment is in support of the underlying theory or the use of the term, or instead, contradicts or rejects that idea. Also, in order to demonstrate how intertwined these terms are within the scientific literature, we recorded when the quote links (at least) two of the three terms together. We categorised the source study of each quote into one of the three main disciplines, social sciences, physical sciences, and biological sciences. This categorisation is predominantly based on the discipline of journal that has published the study as well as the main theme of the study. In most cases, these criteria aligned with one another, but in cases where one single categorisation was not possible, more than one category was assigned to the source article. This categorisation was primarily meant to indicate whether and how the perception of these three terms varies across researchers from different disciplines. The studies that we surveyed had one (or sometimes more) of these three themes as their main focus: modelling, empirical testing, and conceptualisation. We categorised each quote based on the primary category of its underlying study among these three categories. Often more than one category were applicable to the source study of a quote. In those cases, we allowed belonging to more than one category. The purpose of this categorisation was to identify whether there is a noticeable difference in definition and/or perception of our three terms of interest across studies whose main focus is on modelling compared to empirical studies or those that only conceptualise these phenomena. Although this is a somewhat crude categorisation of studies and should be interpreted as such, we suggest that it facilitates some coarse insights. The quotes that we extracted from individual studies were quite diverse. However, we were able to identify common themes across clusters of these quotes. Therefore, to further summarise and categorise these individual quotes, we identified these common themes and added them as short comments to each quote. In cases where the quote did not fit any of those common themes no comments were added to the quote.

The outcome of the analysis outlined above is summarised and reported in Tables 5, 6, and 7, respectively, for terms panic, irrationality, and herding. For each quote listed in these tables, the source reference from which the quote has been extracted is cited. The table also determines whether the quote links each term to either (or both) of the two other terms. It also determines whether the quote offers any definition or conceptualisation on this term (when applicable) and whether it conveys support for the panic/irrationality/herding theory or challenges/contradicts it (when applicable). Then, in order to identify how these characteristics of the quotes are influenced by the discipline from which the study originated, the source reference of the quote is categorised in one (or, occasionally, two) of the three disciplines: social sciences, physical sciences, and biological sciences. The source reference is also categorised based on the nature of the study. If the study is heavily focused on numerical simulation and modelling without much connection to empirical analysis, then it is categorised as a "modelling" type study. If the study presents noticeable empirical components it is categorised as "empirical testing". If the study only offers conceptualisation on this term or its underlying theory, then it is categorised as a "conceptualisation" study. Occasionally some studies had to be categorised in more than one of the two study types.

In order to establish whether 'herding', as a terminology, is suitable and accurate enough for describing the phenomena that it is meant to embody, it seemed necessary to examine this term based on the findings of empirical studies. Therefore, we decided to perform a supplementary survey on the herding phenomenon in evacuation exclusive to the studies that have experimented this question in one form or another. This supplementary survey is not based on the analysis of the quotes per se, rather than concerns the individual studies, those that have provided experimental findings on herding behaviour in evacuations. In collecting a comprehensive set of references related to this supplementary survey, we first extracted relevant studies from a previous review of the empirical studies in crowd dynamics whose reference database was last updated in April 2017 [6]. In order to identify studies that were published after April 2017 we conducted supplementary search in Google Scholar and Scopus, with the main selection criterion being that the experiment report on some form of empirical testing or experimentation on the topic. In total, 24 articles qualified for this supplementary literature analysis. The supplementary analysis allowed us to focus deeper on the herding phenomenon beyond the use of terminology by assembling all existing empirical findings to date. Our conclusions and recommendations regarding the suitability of the term herding are mostly grounded in this secondary analysis.

\section{Quotes on the Term 'Panic'}

The original quotes on the term panic have been listed and analysed in Table 5 in Appendix. The extracted quotes on the term panic were subsequently analysed and after identifying the common themes across the quotes; they were categorised into 22 reduced comments. Table 1 lists these reduced comments along with the frequency of their occurrence in the original comments extracted on the term panic. The table also shows how many times each theme has been repeated in studies across the three different disciplines we considered (i.e., social sciences, physical sciences, and 


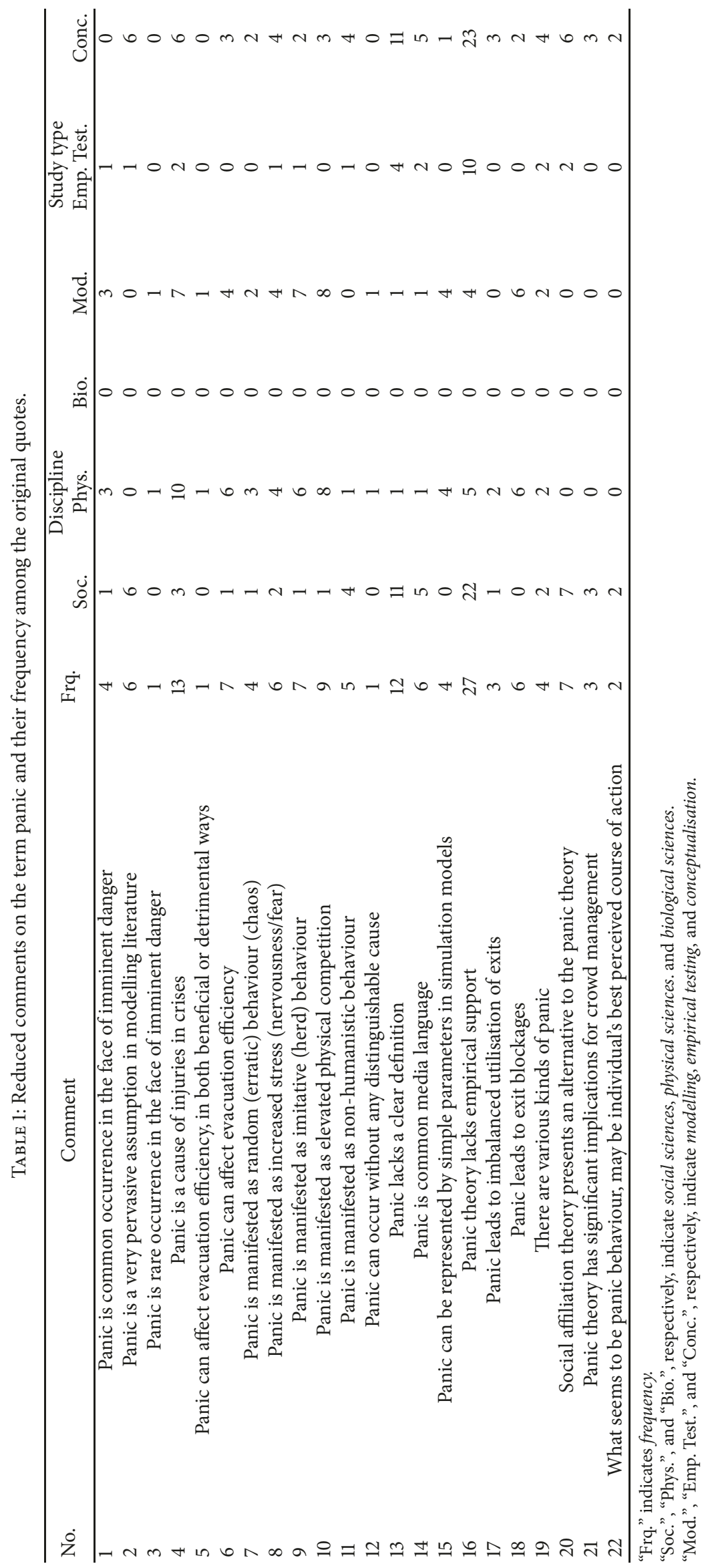



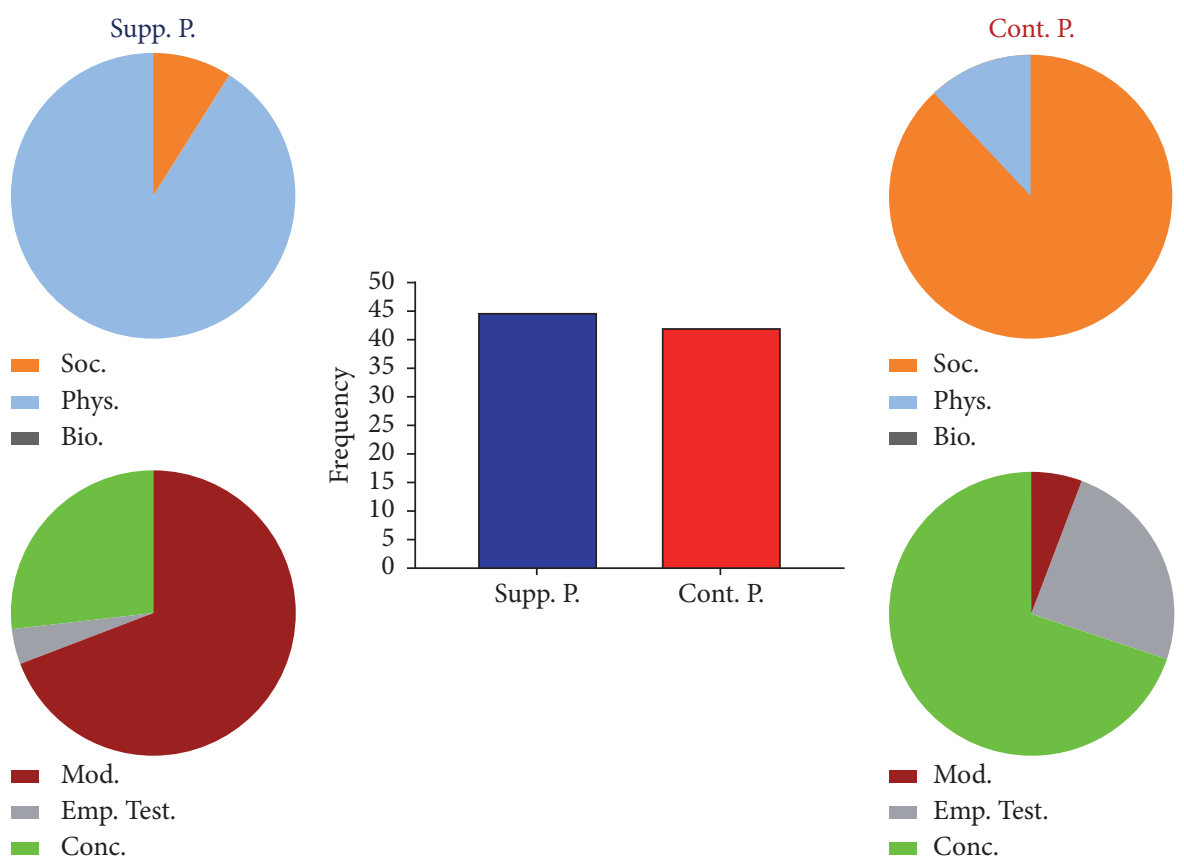

Figure 1: Visualising the frequency of quotes on the term panic that convey support for the theory versus those that challenge it. The pie charts on the left show the frequency of the supporting comments across the disciplines (on the top) and across the study types (in the bottom). Similarly, the pie charts on the right show the frequency of the contradicting comments again across the disciplines (on the top) and across the study types (in the bottom). The column chart in the middle compares the frequency of these comments in total regardless of the discipline or type of the study from which the comments were extracted.

biological sciences) as well as across the three different study types that we considered (i.e., modelling, empirical testing, and conceptualisation). Figure 1 visualises the frequency of the quotes that indicate support for the panic theory versus those that challenge (or contradict) the theory, again across disciplines, and across study types. Figure 2 illustrates the outcome of a temporal analysis on the frequency of the quotes.

One of the most recurring themes in the extracted comments on the term panic concerns the fact that the theory of panic is not well supported by empirical testing $[7,8]$ (comment \#16 in Table 1). Out of nearly 112 comments extracted on the term panic, this theme repeated 27 times. According to Table 1, the majority of such comments originated from studies in the social sciences. Another theme that was very common among the quotes was statements indicating that panic in and of itself is a major cause of injury in emergency incidents and crises and can aggravate the harm caused by the actual crisis $[9,10]$. Quotes of this nature were repeated in 13 cases according to Table 1 (comment \#4) and the majority of the quotes originated from modelling-type studies published within the domains of physical sciences. Third in this ranking was a noticeable set of quotes that pointed out to a major problem regarding the use of panic in evacuation modelling; the fact that the literature has so far not been able to produce a unified definition for the term panic and that has left the theory of panic largely unverifiable and subject to mere speculation and debate $[11,12]$. This comment (\#13 in Table 1) was repeated in 12 cases in the quotes extracted on the term panic and again is one of the areas along which the social and physical science studies divide. The vast majority of the quotes that pointed this issue out were obtained from the social science and conceptualisation studies whereas modelling studies have largely downplayed this problem. This highlights a major problem for modelling practice that aim to represent the so-called panic behaviour in their modelling formulations. In the absence of a clear definition on what panic means, efforts to mathematically represent it in the models will largely be subject to the interpretation of the modeller. In addition, even in the domain of social sciences, panic has a very broad definition ranging from aspects such as extreme emotions, groundless fear, uncontrolled flight behaviour, impatience, the quick transmission of excessive fear (i.e., emotional contagion), or the disappearance of normal social bonds [13]. According to Quarantelli [5], early definitions in sociology textbooks and articles view panic as "the crowd in dissolution" or "collective flight based on a hysterical belief" or "dysfunctional escape behavior generated by fortuitous, ever varying circumstances, but involving impending danger". The author also continues to point out that "early approaches to panic were vague in defining the phenomena. However, most formulations view panic as either extreme and groundless fear, or flight behavior". The inconsistency and the variety of the definitions make the practice of integrating them with predictive models (as aimed by physical scientists working in this domain) more arbitrary and rather subjective.

Of those studies that attempted to offer some definitions on the term panic, we found quotes indicating that panic refers to random, unhinged and erratic behaviour [14] 

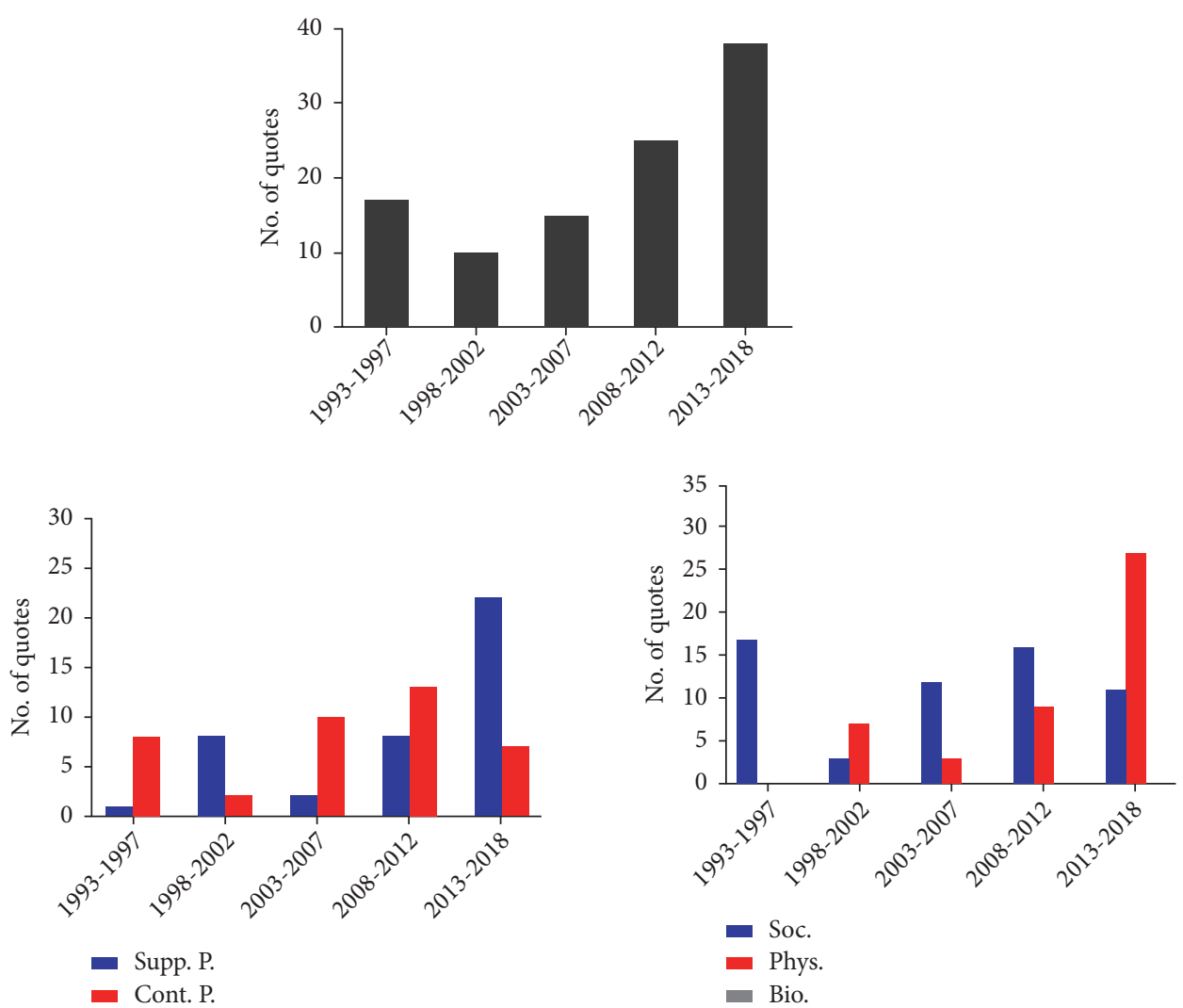

Figure 2: Visualising temporal analyses on quotes that include the term panic. The column chart on the top represents the total number of quotes and the one in the bottom splits the frequency based on whether the quotes support or contradict the theory (chart on the left) and based on the study discipline (chart on the right). To account for the fact that the last time interval includes 6 years as opposed to the rest of the time intervals that include 5 years, the numbers associated with the last interval have been scaled down by a factor of $5 / 6$. The very few studies covered by this review and published prior to 1993 or in 2019 we accommodated in the first and last intervals, respectively.

(comment \#7 in Table 1), comments that referred to panic simply as an extreme state of fear or stress during emergencies [11] (comment \#8 in Table 1), and also those that described panic manifested as nonhumanistic behaviour [15], imitative behaviour [16], or physically competitive behaviour [17]. It is unlikely that all these conditions can exist at the same time which suggests the theory of panic is not clearly defined and has remained so for many years. The mere fact that modellers try to represent panic using model parameters [18] per se contradicts the idea that panic means people showing random behaviour, because something that is completely random cannot be modelled or predicted. Also, the idea that panic is accompanied by an increased tendency to follow the crowd [19] further contradicts the idea of random behaviour, because following the majority is itself a strategy and is not a random act.

The social identity and the affiliative behaviour theory $[13$, 15] proposed by social scientists present arguments against the point of view of the mass panic theory as selfish and uncontrolled behaviour. In contrast to the panic theory, social psychologists have in recent years developed and tested a conceptual model of affiliative collective behaviour in emergencies and disasters that explains how "a sense of common fate is the source of an emergent shared social identity among survivors, which in turn provides the motivation to give social support to others affected". [13]

Similarly, the studies that attribute the inefficiency of crowd evacuation behaviour and the occurrence of exit blockages to the increased physical competitiveness caused by panic have also been challenged by recent empirical work that suggests increased physical competition does not necessarily translate to inefficient egress processes [20-22]. Related to this interpretation (or manifestation) of panic behaviour, Heliövaara, Ehtamo, Helbing, and Korhonen [23] have pointed out that "In the literature of social psychology, the pushing behavior is often related to panic. Panic occurs in situations of scarce and dwindling resources and panicking people tend to behave irrationally and adopt a selfish attitude. However, there has been a consensus for decades that actual panic occurs rarely in real crowds and evacuating people tend to behave rationally".

Another common theme that does not come at the top of the list in terms of the frequency of repeating in the quotes but points to an important problem is comment \#21 which recognises that "panic theory has significant implications for crowd management" [24]. It pertinently reminds us of the implication that the term panic and the assumptions that it implies may have on how managers and emergency 

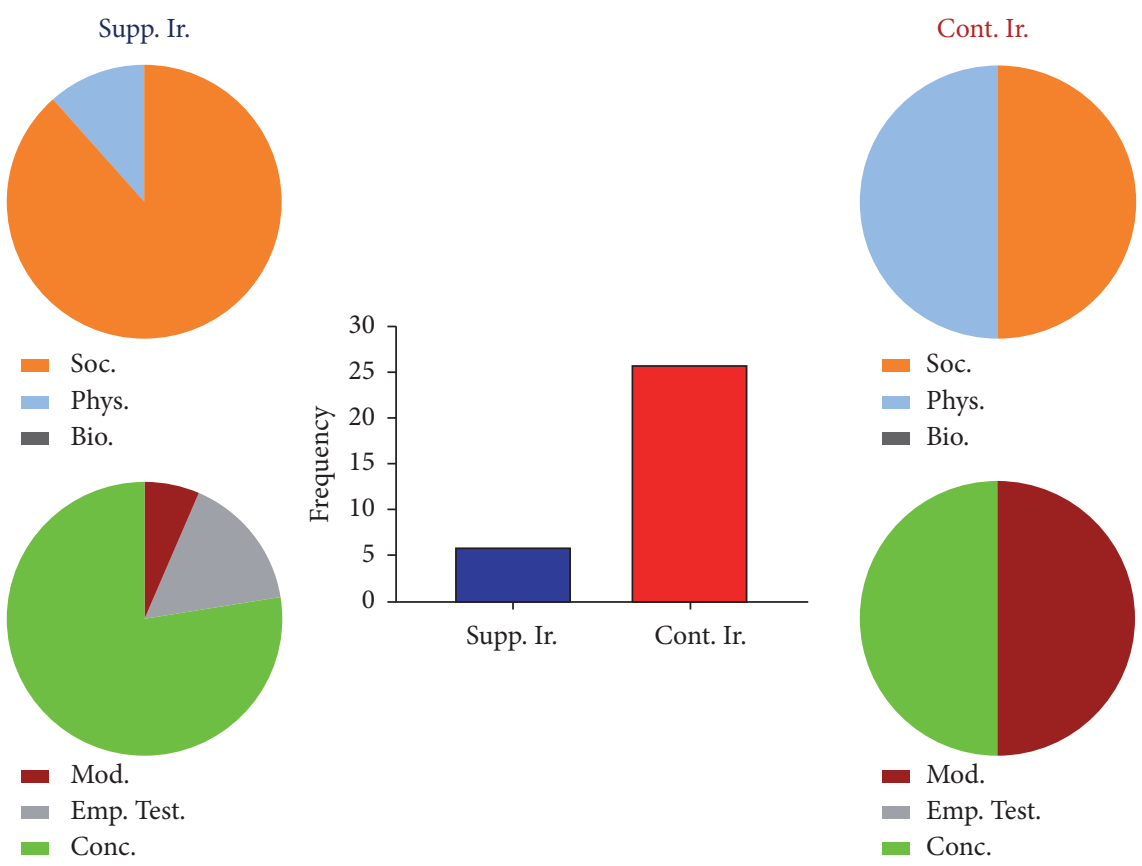

Figure 3: Visualising the frequency of quotes on the term irrationality that convey support for the theory versus those that challenge it. The pie charts on the left show the frequency of the supporting comments across the disciplines (on the top) and across the study types (in the bottom). Similarly, the pie charts on the right show the frequency of the contradicting comments again across the disciplines (on the top) and across the study types (in the bottom). The column chart in the middle compares the frequency of these comments in total regardless of the discipline or type of the study from which the comments were extracted.

responders decide to communicate information to the crowd in incidents of emergency. It recognises that this assumption may be used as a justification to withhold information from the crowd in order to avoid panic and minimise the harm that it may cause. As Heide [25] has pointed out, "The problem with the panic misconception is that the public, the media, and even emergency planners and public officials believe it. Because of this, officials may hesitate to issue warnings because they are convinced that the resulting panic will cause more damage than the disaster itself". He also continues that "this belief has led to recommendations to avoid panic by (1) providing minimal information to occupants in the event of a building fire and (2) carrying on normal activities until the last possible moment". Similar concern has been voiced by Proulx [26] who has stated that "During emergencies, the anticipation of mass 'panic' has been a favoured argument to delay warning the public". This group of studies that pointed to this problem argue extensively that withholding information from potential evacuees cannot reasonably be the best course of action in emergencies $[24,25]$.

The plots presented in Figure 1 provide an illustration of the divide that exists between social science and physical science studies on how they view the term panic. While the quotes extracted in this review show a relatively balanced split in terms of the number of quotes that support the theory of mass panic versus those that contradict it, a clear difference is noticeable when a comparison is made across the disciplines or across the study types. According to these plots, while the majority of the quotes obtained from studies in the domain of physical sciences (mostly, modelling studies) treat the existence of panic as a proven fact, the situation is completely reverse when one considers the quotes extracted from the studies published by social scientists on this topic. Modelling studies have predominantly tried to represent a partial representation of what is known as panic behaviour in their mathematical formulations using simple parameters (that make agents show more noisy behaviour, or more imitative behaviour or more physically competitive behaviour) while assuming panic and its characterisation as proven by their predecessor studies, whereas social scientists have placed a heavier focus on identifying empirical evidence that supports the idea of collective panic behaviour in mass emergencies and have in most cases failed to observe such evidence $[27,28]$.

The temporal analysis presented in Figure 2 further highlights this disconnect between disciplines in how they view the term panic. It further illustrates that, despite the increasing debate on the appropriateness of this term in evacuation literature, the term is increasingly appearing in the scientific literature. According to the set of quotes extracted in this review, while the use of the term among these quotes shows a relatively stable pattern that the social science studies in terms of the frequency of mention, its frequency of being mentioned has surged among the modelling studies. It is also interesting to note, at least among the quotes that were extracted here, that there is no mention of the term panic in physical science studies published prior to year 2000 . 

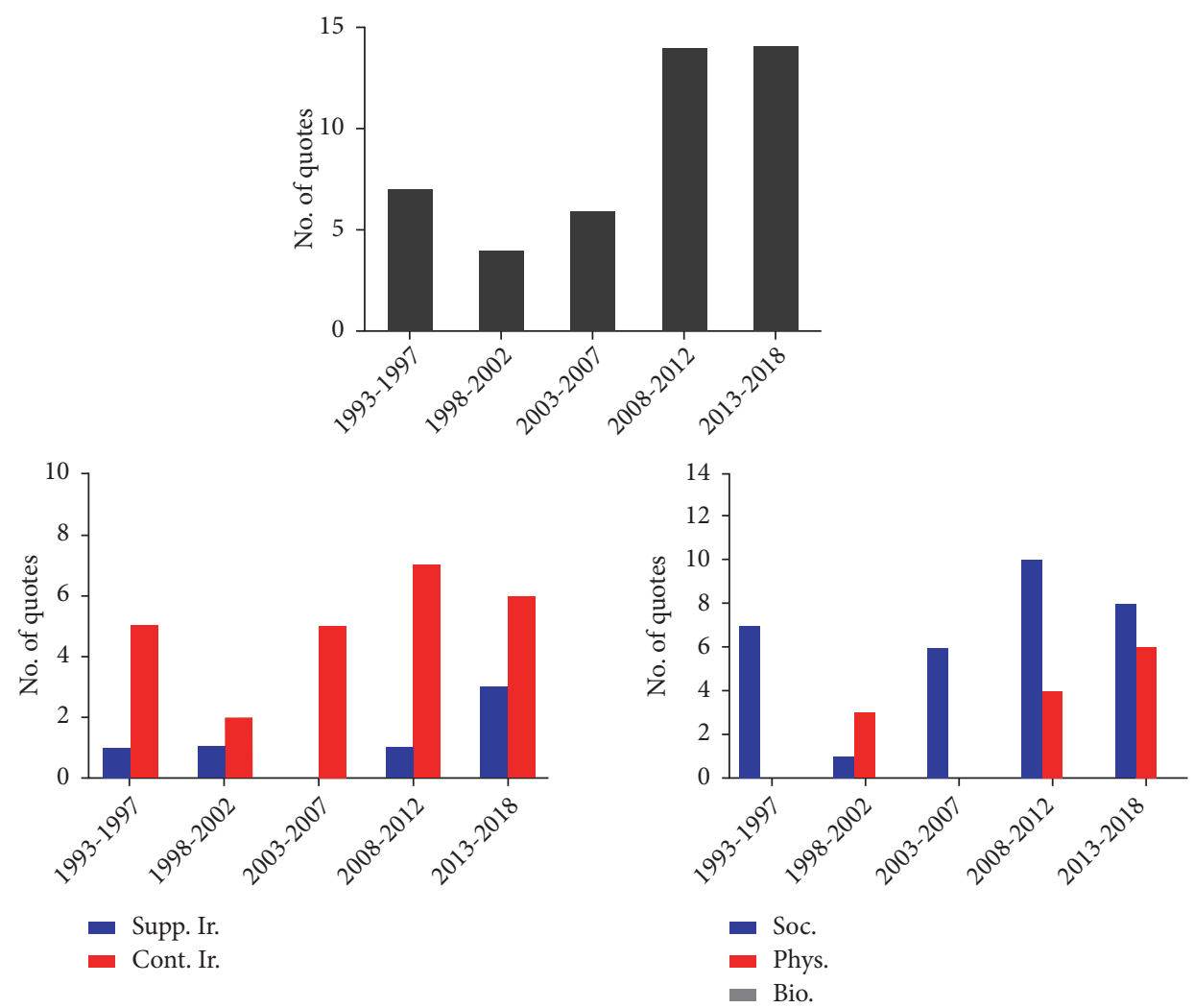

FIGURE 4: Visualising temporal analyses on quotes that include the term irrationality. The column chart on the top represents the total number of quotes and the ones in the bottom splits the frequency based on whether the quotes support or contradict the theory (chart on the left) and based on the study discipline (chart on the right). To account for the fact that the last time interval includes 6 years as opposed to the rest of the time intervals that include 5 years, the numbers associated with the last interval have been scaled down by a factor of $5 / 6$. The very few studies covered by this review and published prior to 1993 or in 2019 we accommodated in the first and last intervals respectively.

\section{Quotes on the Term 'Irrationality'}

The original quotes extracted on the term irrationality are listed in Table 6 in Appendix where similar type of categorisation has been conducted to that of the panic term as explained in the previous section. These quotes were categorised subsequently into 11 common themes presented as reduced comments on the term irrationality in Table 2. Figure 3 provides a visual illustration of the frequency of the comments on the term irrationality based on the total set of comments, the discipline of their origin and the type of their study of origin. And Figure 4 provides the outcome of a temporal analysis on these comments based on the year of publication for their study of origin.

The most common theme that was observable among the quotes that were extracted in this work were those that attribute irrationality very closely to panic, by stating that making irrational decisions is one of the aspects of collective panic (comment \#1 in Table 2) [29]. In other words, these were the comments which suggest that panic implies irrational behaviour too. According to Quarantelli [5], for example, "present day discussions about panic also revolve around whether or not the behavior is irrational, and whether it is highly contagious or not". We also found a relatively substantial number of quotes challenging the theory of irrationality and stating that the theory cannot be regarded as an accurate and verifiable description of a behavioural phenomenon in the face of threats $[15,30,31]$. This comment was the second most common in the list of reduced comments on irrationality (comment \#7).

Another group of statements pointed to a set of very important dimensions which are often neglected in discussions of the topic of irrational behaviour and that includes (1) irrational from whose perspective and (2) irrational relative to which reference point. These statements are collectively reflected in comments $\# 9,10$, and 11 . As pointed out by Drury, Novelli, and Stott [24], "To judge a response as irrational requires a frame of reference, but the frame of reference is often unclear in a mass emergency". Therefore, it is not sufficient to merely talk about the rationality of human responses without measuring the effectiveness of the response relative to a proper reference point and that is an element that is often missing from the discussions on this topic. How such a reference point can be set and how the efficiency or rationality or optimality of behaviour can be measured against it is certainly a matter of research in this area [32], but its necessity seems to be indisputable. Further on that issue, a considerable number of studies that were reviewed pointed out that what seems an irrational act may be an individual's best perceived course of action. Drury, 


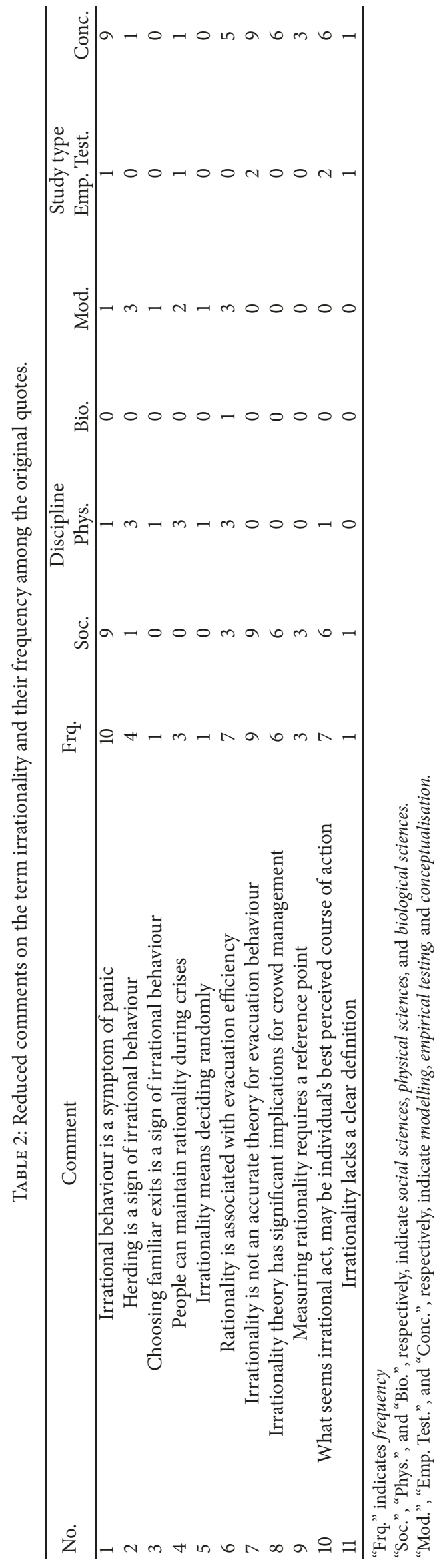


Novelli, and Stott [24] stated that "Fleeing, fear, screaming or other responses to perceived danger may therefore be entirely reasonable [rational] given the limited information - and limited choices - available to people in the midst of an emergency". In a more recent study, Drury [13] further elaborates on the importance of taking into consideration who judges the behaviour as irrational. He points out that "what appears post hoc and from an external perspective to be an overreaction (such as running frantically following a bomb blast) might be reasonable and proportionate from the perspective of those involved". Similarly, Kelley, Condry Jr, Dahlke, and Hill [33] mentioned that "The individual is no less rational or moral in the panic than in any other situation. $\mathrm{He}$ is always in pursuit of his own interests and acts on the basis of his current estimates of where these lie". The comment by Sheppard, Rubin, Wardman, and Wessely [34] stating that "Incorrect decision-making due to incomplete information or insufficient resources is not the same as irrational decision-making and as such is not sufficient to categorise someone as panicking" as well as the conclusion of the study of Heliövaara, Ehtamo, Helbing, and Korhonen [23] stating that "The jams created at bottlenecks along the exit route are often considered to be caused by irrational behavior, a state of psychological panic. However, this study shows that, under threatening conditions, clogging may be caused by crowd members who act rationally according to simple and intuitive assumptions" are also along those lines. Further to that, we also suggest that the research in this area needs to differentiate between what is traditionally known as "social optimum" versus "individualistic optimum" in scenarios where humans interact with one another in their decisionmaking and particularly those in which they compete for limited resources (which is the case in situations of emergency with the resources being the limited capacity for escape) [35]. In such systems, these two types of optimums often do not coincide with each other. What is optimum course of action from an individual decision-making perspective may not necessarily be the optimum behaviour from a system perspective. We suggest that this is another dimension that needs to be considered in conversations on this topic and in moving towards more operational definitions for rationality.

The plots shown in Figures 3 and 4 demonstrate that, similar to the term panic, the use of the term irrationality in studies of evacuation is increasing according to the quotes collected in this work. These figures, compared to Figures 1 and 2, demonstrate that there were lesser numbers of mentions of the term irrationality compared to that of panic, according to the references that we reviewed. However, there is a relatively higher percentage of the quotes that do not support the theory of collective irrationality in escape scenarios compared to the nearly even split that was identified on the term panic (the column charts in the middle). In other words, irrationality appears to be a less popular and less common term in the studies that we surveyed in this review and is cited much less frequently in modelling studies especially compared to the term panic which appears to be more pervasive. We only had a handful of quotes that supported the theory of irrationality, whereas we extracted a relatively considerable number of quotes, 26 quotes, challenging this idea, and those quotes split evenly between the social and physical science studies according to Figure 3.

\section{Quotes on the Term 'Herding'}

The original quotes on the term herding have been listed in Table 7 in Appendix. In addition to the analysis on the quotes that have mentioned this term, a detailed analysis was conducted on empirical studies about the herding assumption in evacuations. Figure 5 provides a visual illustration of the frequency of the comments on the term herding based on the total set of comments, the discipline of their origin and the type of their study of origin. And Figure 6 provides the outcome of a temporal analysis on these comments based on the year of publication for their study of origin.

The most common theme across the set of quotes that we analysed was related to the definition of the term herding in evacuation. According to these quotes, herding in evacuation refers to an increased tendency to follow the crowd, or more specifically to imitate the action of the majority $[36,37]$. This theme was repeated in 15 quotes out of 72 quotes that were identified on this term (comment \#18 in Table 3). Unlike the set of quotes on the term panic and irrationality that did not provide any consensus in terms of the definition and rather added to the mixture on the definition of these terms, the quotes on herding indicated that the majority of studies perceive this term in a roughly similar way. This is of course beside the point of how accurate or suitable this term is for application in evacuation research which is a matter we will discuss below. It merely reflects and describes the current state of the literature and the dominant view on how this term is used and what it refers to.

Another common theme among the quotes we obtained was the use of imbalanced utilisation of exits observed in crowd escape scenarios (regardless of how likely that is to occur) as evidence for herding $[17,38,39]$. This constitutes the reduced comment \#20 in Table 3 that was repeated 11 times across all the quotes. The statements reflected by this reduced comment basically assumed that if the crowd shows an imbalance in the utilisation of exits in spaces where there are multiple exit options, then that can be regarded as evidence that individuals within the crowd tend to copy the action of majority. However, whether this imbalanced use of exit capacities stems from an inherent tendency for copying the action of the majority (that individuals made a conscious decision to follow the crowd) or is attributable to other reasons is a matter of debate which will be discussed in more detail in the following sections [40-42].

A considerable body of studies that we reviewed provided comments that indicate herd behaviour, as a feature of escape panic, is a common form of behaviour in evacuations and thus it should be a common assumption for numerical modelling (i.e., numerical models need to produce herding effect in order to be deemed realistic) [18, 43-46]. These are collectively reflected in reduced comments $\# 1,2,4$, and 6 in Table 3. While we leave examination of the validity of this assumption to our discussion on empirical studies, we only mention here that as opposed to these abundant set of 


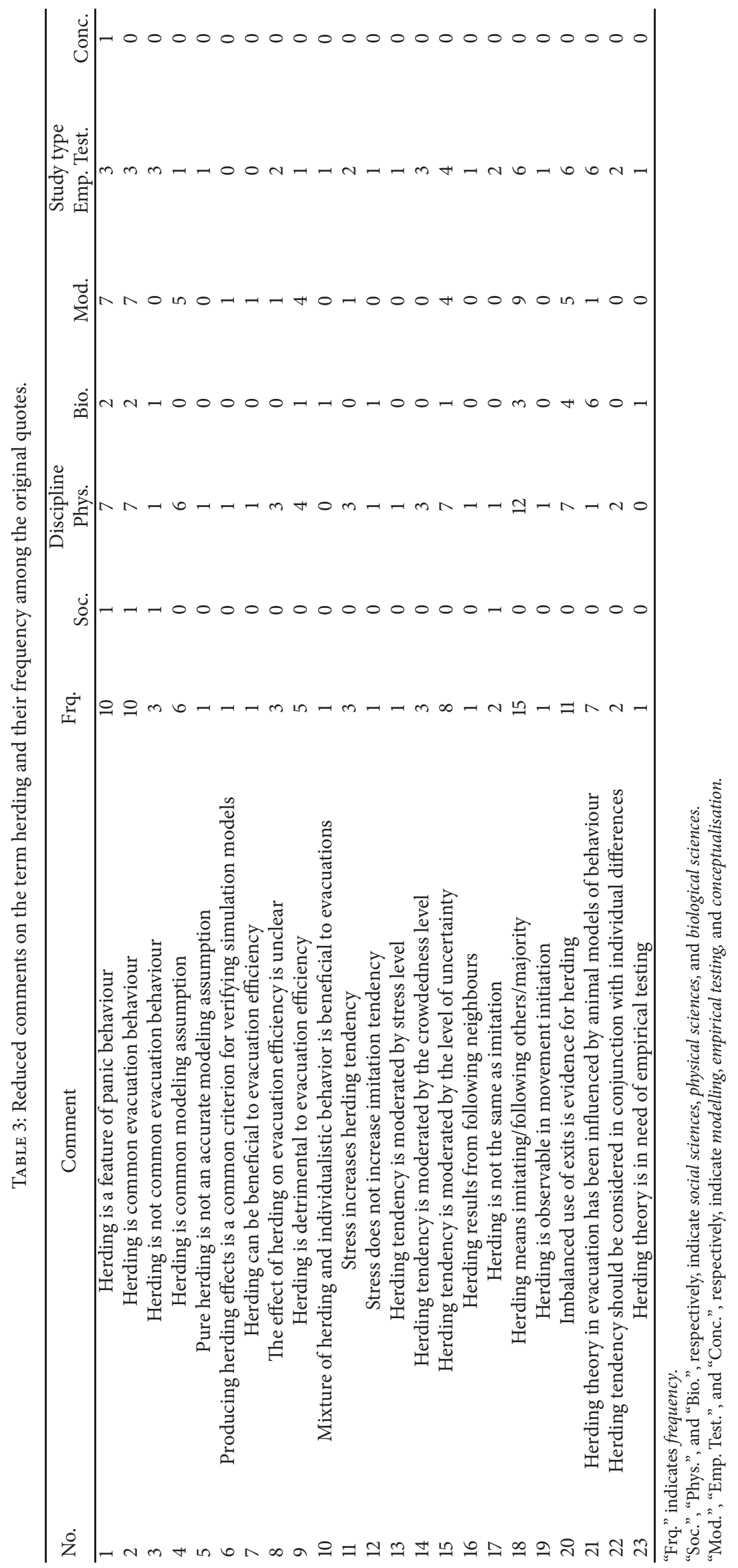



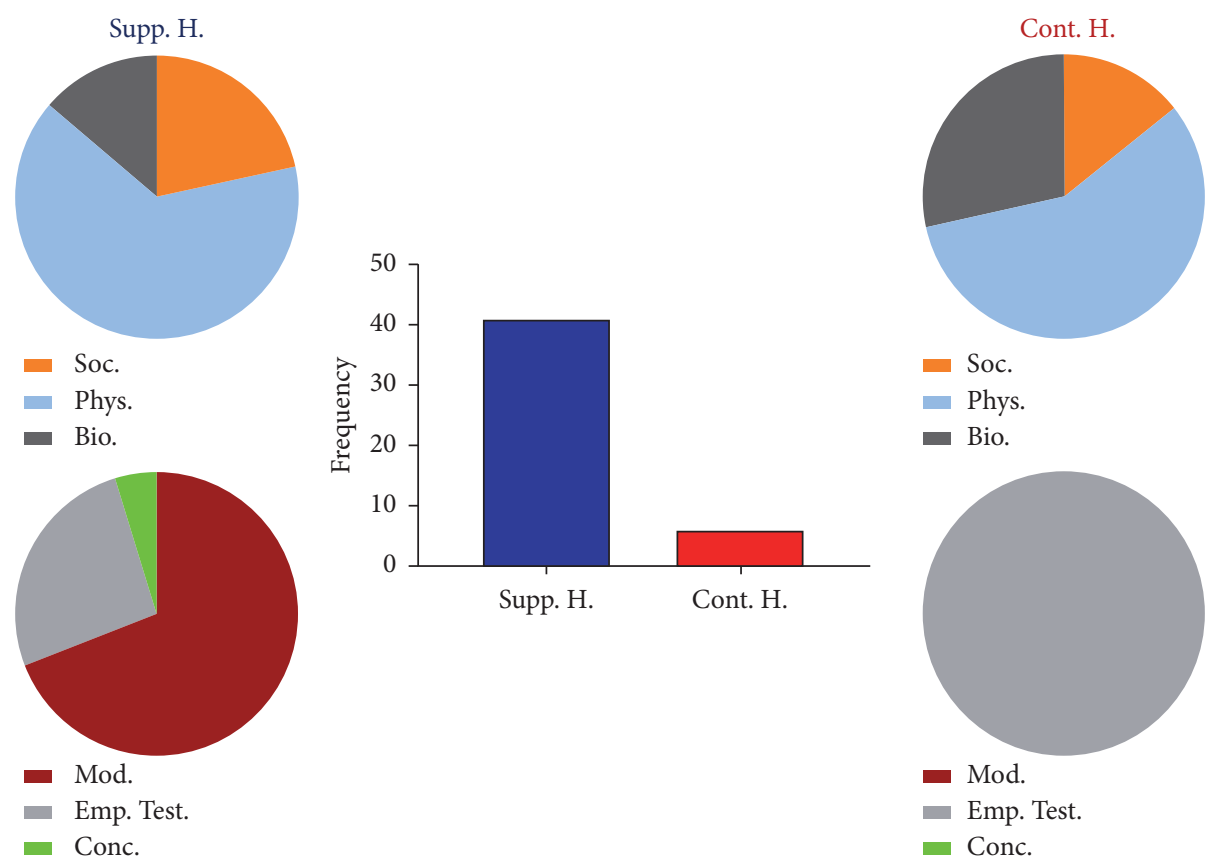

Figure 5: Visualising the frequency of quotes on the term herding that convey support for the theory versus those that challenge it. The pie charts on the left show the frequency of the supporting comments across the disciplines (on the top) and across the study types (in the bottom). Similarly, the pie charts on the right show the frequency of the contradicting comments again across the disciplines (on the top) and across the study types (in the bottom). The column chart in the middle compares the frequency of these comments in total regardless of the discipline or type of the study from which the comments were extracted.

comments, we had quotes that provided a different view and disregarded the assumption that people show herd mentality in escape situations [47] in addition to quotes from studies that recognise that unless people face substantial amount of uncertainty in their surroundings, they will not be likely to take imitative actions [48].

A number of quotes that we extracted considered how herding tendencies influences efficiency of collective crowd egress. These quotes ranged from suggesting that herding behaviour is a detriment to efficient evacuations [37, 49] to those that believe this effect is still unestablished $[39,40,50]$ and that there may be scenarios where herding tendencies are beneficial to an escaping crowd [18]. The subset of these quotes that have not been derived from any simulating testing and are more of a speculative nature did not made it clear which aspect of evacuation decision-making they refer to when connecting herding to the escape efficiency. This is basically a distinction that has not thus far been common in the literature. In line with this question, the phenomenon of mixed strategy (i.e., mixture of herding and individualistic behaviour) has been investigated by several numerical studies. A number of those findings reflect on the findings of such studies. These studies have also contributed a mixture of evidence to the literature with some suggesting that a crowd can benefit from mixed strategies [19] and some suggesting that any percentage of herding strategy within the crowd has a negative impact on the evacuation efficiency [51].

The plots in Figure 5 suggest that unlike panic and irrationality, the herding terminology is a much better accepted term in the crowd dynamics literature. We found many quotes that support this theory and this is far more common among the modelling studies published in the physical science domain. However, the temporal analysis in Figure 6 reveals that firstly, the number of quotes on the term herding shows a surge in the more recent publications and secondly, those that contradict or challenge the herding theory (or the terminology) have only emerged within the last five years and that could be attributable to the rapid increase in the empirical studies within that period many of which observed evidence that did not support this theory [41, 48, 52]

\section{Experimental Findings on 'Herding'}

Unlike the terms of panic and irrationality for which a lack of clear definition was one of the most noticeable aspects of our review, the term herding has a clearer, although largely implicit, definition in the literature. The majority of the quotes indicated that this term is used to describe imitation behaviour or the act of following others. Whether the 'following' specifically means copying the action of the 'majority' was less clear. Nevertheless, given this higher clarity of meaning, the hypothesis of herding behaviour (or as we prefer to say, the role of social influence) has been more operationalizable and this has allowed the hypothesis to be empirically tested in various forms by considerable number of studies mostly published within the last five years. Here, we comprehensively review these studies and their findings to see what we currently know about this behavioural theory. We 

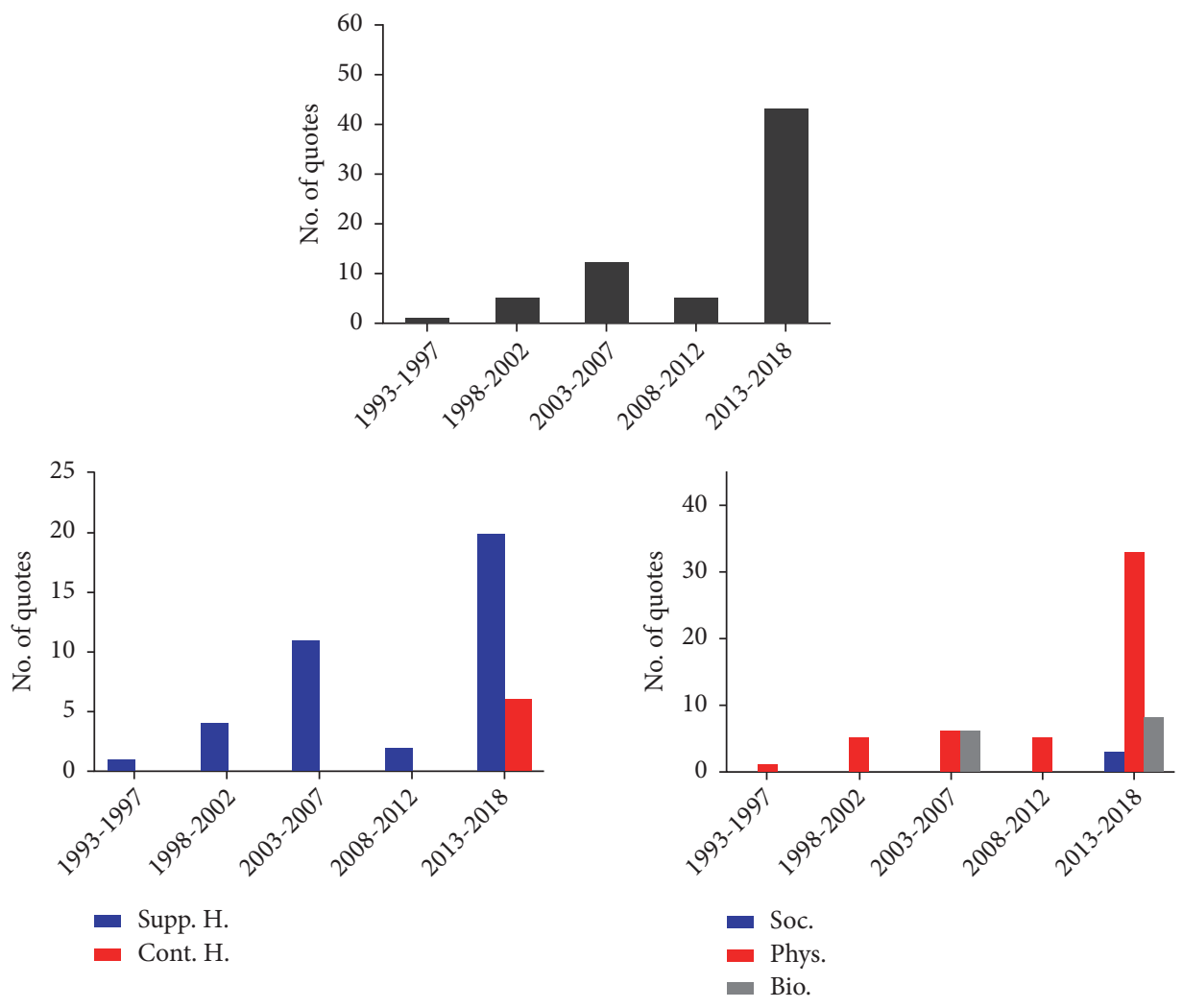

FIGURE 6: Visualising temporal analyses on quotes that include the term herding. The column chart on the top represents the total number of quotes and the ones in the bottom splits the frequency based on whether the quotes support or contradict the theory (chart on the left) and based on the study discipline (chart on the right). To account for the fact that the last time interval includes 6 years as opposed to the rest of the time intervals that include 5 years, the numbers associated with the last interval have been scaled down by a factor of $5 / 6$. The very few studies covered by this review and published prior to 1993 or in 2019 we accommodated in the first and last intervals, respectively.

also discuss the variety of terminologies that have been used to describe this phenomenon along with their implications.

The set of studies that we reviewed often identify as experiments on peer effect, social influence or neighbour effect in evacuations [53-56] and some directly frame the study as an investigation of herding behaviour $[50,57]$. This section provides a comprehensive review of these studies. In total, 24 studies were identified on this topic which have used empirical data of some form. The characteristics of these studies were analysed and subsequently summarised in Table 8 in Appendix. This table shows four main aspects or dimensions of each of these studies: (i) what aspect of the evacuation behaviour was investigated in relation to the peer effect, (ii) what method they used for their data collection (this could be virtual-reality, real crowds, or nonhuman crowd experiments), (iii) did the study find evidence of herding effect (which according to the majority of the body of studies, refers to imitative behaviour), and (iv) what is the main interesting aspect of their findings (this part is provided as a short comment alongside each reference). This analysis is the first to officially recognise that studies and discussions on herding in evacuation should be performed in relation to specific aspects of evacuee's decisions as opposed to discussing the topic in broad terms such as whether people generally show an amplified tendency towards mass behaviour (in all aspects of their decision-making). We have identified and reported the specific aspect of the decisionmaking that has been investigated in connection with peer influence for each of the studies listed in Table 8 .

6.1. Definitions and Alternative Terminologies for Herding. As mentioned previously, the problem has been framed using a range of terminologies such as imitation [49], allelomimetic behaviour, or allomimetic behaviour [46] (defined as a range of activities in which the performance of a behaviour increases the probability of that behaviour being performed by other nearby animals), social influence $[54,55]$, peer behaviour effect [53], neighbour behaviour effect [56], followthe-crowd behaviour [48], and of course, herding or herdtype behaviour $[41,50,52,57,58]$. The phenomenon is also referred to by a substantial body of studies as "symmetry breaking" [42, 59-62]. From a linguistic perspective, however, the term does not exactly equate imitation. According to the Longman Dictionary [63], the verb "herd" means "to bring people together in a large group" or "to make animals move together in a group". However, as shown in the previous section, the term is used almost as a substitute for "imitation" in the crowd dynamics literature.

As a pioneer study in the field of crowd dynamics Helbing, Farkas, and Vicsek [19] discussed the phenomenon and 
introduced it to numerical simulations. In their conceptualisations "pure herding behaviour implies that the entire crowd will eventually move into the same and probably blocked direction, so that available exits are not efficiently used". These numerical testings were conducted in relation to a simulated room with two exits. Therefore, we assume that the term herding in its original form was specifically used in relation to exit choice behaviour. And this is in fact a common characteristic of the main body of studies that have so far investigated the herding assumption using empirical methods. They predominantly interpret herding in the context of exit choice making. However, the literature has been increasingly recognising the role of social influence in other aspects of evacuation decision-making and a few studies have looked into this problem in connection with reaction responses of evacuees [55, 58, 64] and exit choice adaptation (or exit choice changing) behaviour $[65,66]$ of evacuees. Hence, in our analysis of the 24 empirical studies on this topic, we have categorised each item into one (or occasionally two) of these three categories: exit (direction) choice, exit (direction) choice changing, and reaction times. We also identified four general experimental methods that have been adopted to study this topic: human crowd (laboratory or evacuation drill) experiments, virtualreality experiments, experiments with groups of ants, and experiments with groups of mice (as analogical experiments of human crowds).

In the following subsections, we first investigate the origins of the term herding in crowd dynamics and review the first experiments (predominantly based on social insects) which referred to this notion as the 'symmetry breaking' phenomenon. We subsequently review the findings of empirical studies that investigated the role of the social influence in relation to each of the three behavioural sublayers that identified earlier. We then discuss two questions in subsections that follow: (1) can observations of herding with social insects or animals be reliably extrapolated to humans and (2) is the term herding itself a suitable terminology to be used in crowd dynamics.

6.2. Herding and Symmetry Breaking. The first attempt to empirically test the herding assumption in the context of crowd escape dates back to 2005 (five years after the publication of the pioneer paper in Nature [19]) where a study published by American Naturalist reported on observing "symmetry breaking" effects in experiments with groups of ants [62]. According to the authors, "The phenomenon of herding is a very general feature of the collective behavior of many species in panic conditions, including humans" and this statement constitutes the main premise of their study. The authors observed in this work that groups of ants confined in a chamber show an elevated level of imbalanced exit utilisation when repelled by an aversive stimulus (a certain dose of repellent chemical) and inferred that as a sign that herding phenomenon exists in collective escape scenarios and that the behaviour is shared across a range of species including humans: "Our experimental results, combined with theoretical models, suggest that some features of the collective behavior of humans and ants can be quite similar when escaping under panic." Another statement that the authors have made in their study is that "It has been predicted theoretically that panic induced herding in individuals confined to a room can produce a nonsymmetrical use of two identical exit doors". In evaluating this statement, we argue on a major factor that seems to have been neglected and that is the differentiation between exogenous and endogenous modelling assumptions in numerical simulation methods. The assumption of herding in Helbing, Farkas, and Vicsek [19] was clearly an exogenous assumption meaning that the authors formulated and imposed this assumption in the formulation of their numerical model. Clearly, when one formulates a certain type of phenomenon in the form of mathematical models and implements that model, observing that phenomenon (formulated exogenously) cannot reasonably be regarded as a proof of that phenomenon. We believe that this is a distinction that in a number of cases like this the literature has failed to make when concluding from numerical studies in this field in general. The conclusion from this study has also been cited as an evidence that greater levels of stress and urgency make humans to be more inclined towards imitating the majority's action in an emergency escape context.

The assumption and terminology of symmetry breaking were subsequently followed up by further studies that adopted the ant experiment technique and often made variations to the type of the aversive stimulus [61]. This includes the study of Chung and Lin [59] where using controlled heat-induced aversive stimulus, they observed that the degree of asymmetry increased linearly with the temperature, and also the study of Li, Huan, Roehner, Xu, Zeng, Di, and Han [60] who investigated the effect of density on the extent of symmetry breaking and observed that the degree of asymmetry increased then decreased by ants' density. The most recent study of this kind has shown that symmetry breaking is associated with the difference in the width of exits in proportional ways, thereby concluding that there are, in fact, some patterns of symmetry in symmetry breaking phenomenon in ant groups [42].

\subsection{Herding in Movement Initiation. Laboratory crowd} experiments in virtual and real(istic) environments have increasingly furthered the knowledge on the role of social influence within the recent years [67]. The problem of premovement time in particular has received attention in this context. According to Bode and Codling [68], "Social influence occurs when individuals respond to the behaviour of others and it is an important factor that needs to be considered in research on premovement times in evacuations". The virtual-reality experiments of Kinateder, Müller, Jost, Mühlberger, and Pauli [55] and Van den Berg, van Nes, and Hoogendoorn [58] have both provided evidence on the significant role of peer behaviour effect on reaction to threat (or movement initiation) responses of evacuees. They have shown that the presence of passive virtual agent made subjects delay their movement reaction, the more people someone sees leaving, the more inclined this person is to leave, and that seeing people leave has more impact 
than seeing people stay. The two experiments have been conducted at different levels of virtual crowd density and they collectively suggested that evacuees' reaction to an emergency signal is impacted by their neighbours' behaviour and the direction of influence is towards taking imitative actions, regardless of whether or not the crowd in dense. In relation to the premovement time response, we only know of one study in nonvirtual experimental setting and that is the study of Nilsson and Johansson [64] who utilised the data from an evacuation drill in a cinema. According to Galea, Deere, Hopkin, and Xie [69], "a subset of data from these trials was later analysed to explore the impact of social influence of close neighbours on response time" and "the authors did report that response time for an individual was related to that of a neighbour, so that participants acted more like their neighbours than to others". They concluded from their analysis that social influence is an important factor in reaction time, especially when cues about dangers are unclear, and that social influence (on reaction time) increases with decreasing distance between visitors.

In terms of the influence of imitation in movement initiation on evacuation efficiency, we do not know of any study that has empirically tested this question, but a recent numerical study has shown that lesser variability in reaction times (which could be achieved when individuals tend to initiate their movement as soon as their peers/neighbours do so) shortens the duration of the evacuation [70]. And this has been shown to be the case across a variety of density levels (up to extreme densities). This suggests that herding in movement initiation could be a beneficial form of behaviour (although we should mention that numerical evidence to the contrary of this finding also exists [71] suggesting that a "staged" evacuation strategy (or waiting strategy) could be more efficient than instant collective response).

6.4. Herding in Exit Choice. As mentioned earlier, a significant portion of the empirical knowledge on the role of social influence has been obtained from experiments that investigated exit choice behaviour. The experiments reported by Bode and Codling [52] adopted a simplified form of virtualreality setting in which the subjects have a top-down view of a two-dimensional computer-simulated crowd evacuation scene and control and navigate their simulated agent using mouse clicks while interacting with simulated agents. The setting of this study simulates relatively dense crowd escape scenarios. No distinct pattern of herding behaviour was observed in this study. Experiments of direction/exit choice in three-dimensional forms of virtual-reality have been reported in $[54,55,72,73]$ where the experimental setting often simulated a not-heavily crowded scene. As indicated by the analysis in Table 8 , these studies have generally found evidence for social influence in the direction of imitation. The virtual-reality exit choice experiments reported by Lovreglio, Fonzone, dell'Olio, and Borri [50] have been framed and analysed in the form of discrete choice experiments and represent relatively dense crowds. Using mixed logit models, the authors estimated the relative importance of different factors on exit choice. Their findings suggest that on average social influence, measured as the number of people at exits, reduces the likelihood of exits being selected. Therefore, this study suggests that social influence has an effect, but that the effect is the opposite to what is commonly proposed under the herding assumption. The findings in this work also qualitatively match those reported in [74], derived from an independent discrete choice survey, which again does not support the herding assumption. Another aspect that is shared between these two studies and also the virtualreality studies of Kinateder, Comunale, and Warren [56] and Bode, Wagoum, and Codling [75] is that they have all produced evidence that suggest exit choice making is a multiattribute trade-off (between time-dependant and timeindependent factors [75]). While peer behaviour appears to have significant effect on evacuees' exit decision, it is also traded off with a range of other factors.

These findings have demonstrated that one cannot assume that peer behaviour is the sole determinant of exit choices and that is one of the main reasons we suggest that the term herding may not be the most suitable terminology to be used in this context. First of all, it indicates, by implication, that the influence of observing peer behaviour is always to the direction of imitation (whereas, sometimes the opposite is the case) and secondly, it dismisses the role of other contributing factors that compete with peer behaviour effect. It implies a decision-making mechanism that is predominantly governed by social influence. The overall message of the virtual-reality experiments has been that in not-heavily crowded scenes social influence acts to the direction of imitation and in heavily crowded scenarios the direction of influence largely reverses. But in all those cases, one also needs to take into account the effect of other contributing attributes to the decision-making (other than social influence) as well as the role of individual differences in perceiving the social influence [76].

Recent experiments conducted using crowds of volunteers, particularly those from which individual-level exit choice observations were extracted [48], generally confirm the findings of the virtual-reality experiments discussed above. Particularly, the presence of multiattribute trade-off between a set of factors that include peer influence appears to be a recurring theme in all those studies [77]. In highly dense laboratory crowd experiments, the dominant pattern of exit choice behaviour has been avoiding the majority [65]. However, Haghani and Sarvi [48] have shown that when attribute ambiguity is introduced, the peer behaviour can act at a positive direction (meaning people tend to perceive direction chosen by majority more positively or at least, less negatively in relation to the alternatives for which attribute ambiguity exists). Therefore, it has been suggested that the influence of peer behaviour in evacuation contexts is moderated by the extent of decision uncertainty that evacuees face.

In a recent study, Haghani and Sarvi [41] tested the effect of urgency level as well as the density level on the perception of peer behaviour and the results overall suggested that none of these factors lead to an increased tendency to imitate others. Under higher levels of simulated urgency or when faced with a larger total number of people, decision-makers became 
actually less likely to follow the direction chosen by the majority. In terms of how imitation in exit choices influences egress efficiency, we currently only can resort to the evidence from numerical simulations that suggest any elevated degree of imitation in exit choice making negatively influences total evacuation times. The suggestion from numerical studies is that, when familiar with the location of exits, a crowd of evacuee is best off avoiding a follow-the-majority strategy [49].

6.5. Herding in Exit Choice Adaptation. The empirical evidence on the role of peer effect in how evacuees change/adapt their decisions is very sparse. The topic of decision adaptation [78-80] within the general framework of evacuee's decisionmaking [81] is in general highly underrepresented in the crowd dynamics literature. In particular, when contrasted with the growing body of studies that have experimented exit choice behaviour within the recent years [56, 75-77, $80,82-85$ ] very little attention has relatively been paid to the mechanisms of exit choice changing. Proportionately, much less is known about the influence of peer behaviour on this aspect of evacuee's behaviour compared to the influence on exit choice. Recent studies that have experimented this problem, however, have shown that, in crowded evacuation scenarios (where queues form at exits), observing other people changing their exit decisions is a trigger for the observer to change the initial decision and imitate that action $[65,66]$. It has been shown in these experiments that once one evacuee decides to leave a queue formed at an exit and join another queue at another exit, it increases the likelihood of decision changing by others followed by a burst of decision changes. This phenomenon, however, even though it indicates imitation, is not precisely consistent with a definition of herding as "following the majority". It is consistent with a definition of herding as "imitating others" but "others" in this case are often the minority. In such scenarios, at any point in time, there are more people not changing their decisions compared to the number of individuals who decide to change their initial choice. Numerical testing in a recent study [86] has also been shown that certain degrees of imitation in exit choice making enhances the efficiency of crowd evacuations from a system perspective.

6.6. Herding and Extrapolation of Behaviour from Social Insects and Animals to Humans. The findings of the experiment reported by Haghani and Sarvi [41], as outlined earlier, may be regarded as evidence opposite the symmetry breaking. The experiment showed that as urgency increases, people show even less tendency to follow the direction chosen by more people. The stark contrast between this experiment and those of the symmetry breaking experiments with ants could be worthy of note. The symmetry breaking phenomenon has been proven with ants through several independent experiments. However, recent evidence is overwhelmingly suggesting that the phenomenon does not seem to be replicable when tested with humans. This might be only one of the areas where the escape behaviour of insects and humans differ fundamentally and thereby, generalisation across the two should be avoided [87].
An implication of identifying such inconsistent observations between collective escape behaviour of insects and humans may be that, wherever possible, behavioural experimentation in this domain should take place with humans as opposed to alternative animals/insects as proxies for humans. In some research the notion can arise that findings from research using social insects can be extrapolated directly to emergency evacuations involving humans. However, there are fundamental differences between species that go beyond obvious physical distinguishing factors. For example, the genetic make-up of ant colonies is largely homogeneous which is likely to affect the trade-off between individual survival and survival of other colony members. This could explain why entire ant colonies reenter previously evacuated nests in an attempt to save their brood (D. Parisi, personal communication), behaviour that is unlikely to occur at this scale in humans.

An argument in response to our proposition is that such experiments are often conducted to help us replicate the sense of real danger which cannot be possibly considered in experiments with human subjects. It should, however, be noted that in many cases, proxies for lifethreating dangers, such as creating the sense of urgency using monetary incentives, could be used within the frameworks of ethical experimentation and without imposing any real danger on participants. This possibility could be taken into consideration as offering a trade-off between using a proxy urgency-inducing treatment with real humans (an accepting a certain level of contextual approximation) as opposed to using real urgency-inducing stimuli with animals/insects (and accepting their fundamental behavioural differences as a very different kind of approximation).

6.7. Is 'Herding' an Accurate Terminology? Previous discussions in Section 5 revealed that the term herding is being used in the literature with lesser degrees of inconsistency in terms of the definition, compared to the terms panic and irrationality. According to the quotes that we extracted, most authors use this term as a reference to the act of (blindly/passively) following others. There are alternative interpretations as well, such as 'synchronisation of actions' or 'congregations of people' or 'large groups moving to the same direction'. But these definitions are not as common as 'copying' or 'imitation' or 'conforming to the behaviour of the neighbours or the majority'. However, in light of the empirical findings that we reviewed in this section, here we argue that, despite this relative consistency in definition, the term herding per se lacks accuracy in conveying the meaning that it is meant to embody.

Firstly, herding is a term that has been originally used in relation to animal groups. In that sense, it implicitly coveys an irrational collective unconsciousness where individuals surrender their own wisdom to the group and copy the group blindly (thus, by a stretch of meaning, it may implicitly convey the meaning of 'acting like a group of animals'). In that sense, the term is indeed linked to the panic/irrationality theory which our review suggested to be not so well supported. A change of terminology may help dissociate this concept from panic/irrationality. Further, the mere use of the 
term herding in the scientific literature gives the indication that there are similarities between the escape-from-danger responses of humans and those of animals, thereby, justifying experimentation of animals'/insects' behaviour as a proxy for that of humans. As we discussed earlier in Section 6.5, the emerging empirical evidence has not produced much promising evidence for such analogies. Secondly, our review of empirical findings showed that people exhibit various kinds of tendency towards copying or not copying the actions of others in evacuation contexts. Their behaviour appears to be rather complex. For certain aspects of their behaviour (or under certain contextual circumstances), they show tendency to avoid the action of the majority rather than follow. Also, in some cases, they might show imitative tendency but towards the action of the minority rather the majority. The literature is clearly showing that social influence on evacuation behaviour differs depending on the type of action (e.g., movement initiation, direction choice, and decision changing) and also, depending on certain contextual factors (e.g., how crowded the space is and how familiar the occupant is with the surrounding environment), not to mention the role of individual differences in all that. Therefore, there is a great amount of nuance involved in this phenomenon that the term herding fails to capture. The term gives the indications that when we talk about the social influence, we essentially mean 'following others', whereas, the term social/peer/neighbour influence itself maintains neutrality and flexibility in that regard. It embodies both tendencies to follow or to avoid others, as well as tendencies to follow the majority or the minority. For these reasons, we suggest that while the idea behind exploring the role of social influence in evacuation is legitimately valid and even essential, the problem does not need to be formulated as a question about herding. We argue that this term comes with an unnecessary amount of predisposed connotation (partly inherited from the panic theory) as opposed to the nuance, neutrality and flexibility that is required for describing a rather complex phenomenon like this.

\section{Discussion}

We have adopted a literature survey approach to investigate, in an open-minded way, if preferred or dominant definitions for the three terms we investigate have emerged over time in the literature. While we cannot claim that our literature search is completely exhaustive, we argue that the number of publications included is sufficiently large to adequately support our findings. We acknowledge that the way we have prioritised comments on the terms we investigate within papers and the way we have grouped or reduced comments and categorised supportive or unsupportive comments, as well as the disciplines that publications belong to, is to some extent subjective. We hope that this qualitative analysis is nevertheless a useful synthesis of the complete body of comments we found which we report in full in the Appendix, Tables 5-7. Given the ambiguity/inaccuracy that we found regarding the use of these terms and the lack of empirical evidence for them (except for "herding" which is comparatively a betterdefined concept), it was not possible to perform a quantitative meta-analysis or metasynthesis on the evidence pertaining to "panic" and "irrationality". As the empirical base for research into human crowd dynamics continues to grow [6], such meta-analyses will become an attractive option to test the support for specific hypotheses by incorporating evidence across several studies in a similar way to what has been done in other fields of research [88]. However, we anticipate that such an analysis will not be possible for the three terms we discuss here. The unification of behavioural terminologies and hypotheses could be a major useful step towards shaping the literature in that direction.

Our survey of the crowd dynamics literature illustrated that the three terms that we reviewed do not have an unequivocally accepted definition in the literature. This is particularly the case for the terms panic and irrationality. While these terms are still used in increasing numbers of publications, they are also discussed controversially. And in the case of "irrationality", most publications are explicitly critical of the use of this term. An additional and complicating aspect suggested by our literature search is that the terms are used and treated differently in studies from different broad disciplines of research. This is particularly evident for the term "panic" which seems accepted and used (albeit in different ways) in studies which we classified as belonging to the physical sciences but is mostly opposed in studies we classified as belonging to the social sciences. Based on this, we suggest that at present, the use of the three terms "panic", "irrationality" and "herding" in the scientific literature does not contribute constructively to describing, understanding or even predicting evacuation behaviour.

A recent multidisciplinary effort to define terms frequently used in research on pedestrian dynamics does not include definitions for the terms "panic", "irrationality", and "herding" [89]. Instead, this glossary even includes the suggestion that some terms, including "panic", and "herding" that lack a clear definition or could lead to misunderstandings should not be used. This is in line with what we have found by searching the literature extensively for uses of these three terms, as well as the suggestions of several authors in the field of social psychology. As Quarantelli [5] already concluded in a seminal study titled "The sociology of panic" in 2001, "There are two questions that will loom even larger in the future. One is why despite the research evidence, the idea of "panic" captures the popular imagination and continues to be evoked by scholars of human behavior. A second basic question is whether there is still any scientific justification for the continuing use of the concept in any technical sense in the collective behavior area". Our review suggested that the use of these terminologies has not constructively contributed value to the evacuation dynamics literature and if anything, in some cases, the clear lack of definitions for (at least two of) these terms has ambiguated the research field and hampered the efforts of the researchers. Having reviewed the use of these terms, for example, we were not able to identify a definition for the term panic that can be framed as a testable hypothesis. As a result of this issue in this research domain, assumptions have been made that can neither be verified not rejected and computational prediction models have been formulated that cannot be objectively validated. 
These issues do not imply that anything loosely related to the three terms cannot be investigated systematically. Our detailed investigation of empirical evidence related to the term "herding" suggests a constructive way forward. While herding is arguably a vague concept, researchers have specified concrete behavioural phenomena instead, such as imitative behaviour, that lend themselves to scientific investigation via observations, experiments or models. In a similar vein, instead of focussing on the high-level ambiguous term "panic", we suggest it is a legitimate question to ask "how intense levels of urgency, stress or fear influence evacuation behaviour", "how optimality of evacuees' decisions can be measured, quantified or improved", "under what circumstances evacuees make more suboptimal decisions", "how observing peer behaviour influences various aspects of evacuee's decisions" or "under what circumstances evacuees are more/less inclined to imitate actions of others". Importantly, framing these questions in the form of ambiguous terms, such as "panic", "irrationality", or "herding", may act as an impedance in scientifically investigating the topics broadly related to the terms by obfuscating an otherwise operationalizable set of questions. In particular, the imprecise assumptions that can accompany these terms may dissuade or divert research from studying these phenomena at the level of nuance that they require. Therefore, we argue that it would be beneficial for the progress of research in this field that the questions related to the three terms discussed here are clearly stated in terms of verifiable hypotheses and be operationalized for empirical testing.

As an illustration for why the language that is used to describe behavioural phenomena in this context matters and can potentially have a significant influence on shaping and directing the research in this field and even management practices, consider the following examples. The assumption that phenomena related to the term panic are not testable in experimental settings with humans has made many authors favour pure numerical methods over experimentation or favour experimentation with animals or insects over experiments with human crowds [59-62, 90-97]. In terms of management practices, the theory could be cited in crises situations as a reason for withholding information from the crowd by managing authorities in order to save more lives. According to the studies that we reviewed, this is based on the rationale that if people know about a critical situation, it might agitate them, ultimately causing them to panic which will lead to irrational behaviour. In contrast to this line of thinking, several authors like Heide [25] have argued that "Evacuation warnings should not be withheld or delayed for fear of precipitating widespread panic". Similar important implications are also conveyed by the term herding. The term, as we showed in our detailed analysis of quotes, has largely been used in the literature to convey imitative type of behaviour [49]. However, the use of this (largely animalistic) term does not make it clear whether there will be contexts or aspects of behaviour in which people do not tend to imitate. It also depicts a mechanism of decision-making in which peer influence is the only factor or the dominant factor while trivialising the role of other potential contributing factors to human responses.
The research on evacuation dynamics has been actively in progress for several decades. Many scholars from a range of disciplines have been researching this topic and significant progress has been made. However, we argued that, if thus far, this ample effort has not converged to any well-defined and empirically supported characterisation or a well-accepted numerical model for panic, then it may be unlikely that such goal be achieved in the future. This may be an indication that some parts of the literature in this field may be in need a fundamental reformulation. It warrants that some of the concepts or terminologies, including those studied in this review, be revisited and replaced with more proper substitutes. In conclusion, we suggest that instead of framing their investigation under the umbrella of the frequently used, but ambiguous terms, "panic", "irrationality", and "herding", researchers could simply state the precise assumptions or hypotheses underlying their work. In doing so, a more integrative approach between the numerical, empirical, and social science studies could prove useful. Table 4 lists a summary of the conclusions that we drew based on this review regarding the use of each of the three terms, along with our recommendations.

\section{Appendix}

\section{A.}

See Table 5.

\section{B.}

See Table 6.

C.

See Table 7.

D.

See Table 8 .

\section{Conflicts of Interest}

The authors declare that there are no conflicts of interest regarding the publication of this paper.

\section{Acknowledgments}

Alessandro Corbetta acknowledges the support of the Talent Scheme (Veni) research programme, through project number 16771, which is financed by the Netherlands Organization for Scientific Research (NWO). E. Cristiani would like to thank the Italian Minister of Instruction, University and Research (MIUR) to support this research with funds coming from PRIN Project 2017 (no. 2017KKJP4X entitled "Innovative numerical methods for evolutionary partial differential equations and applications"). 


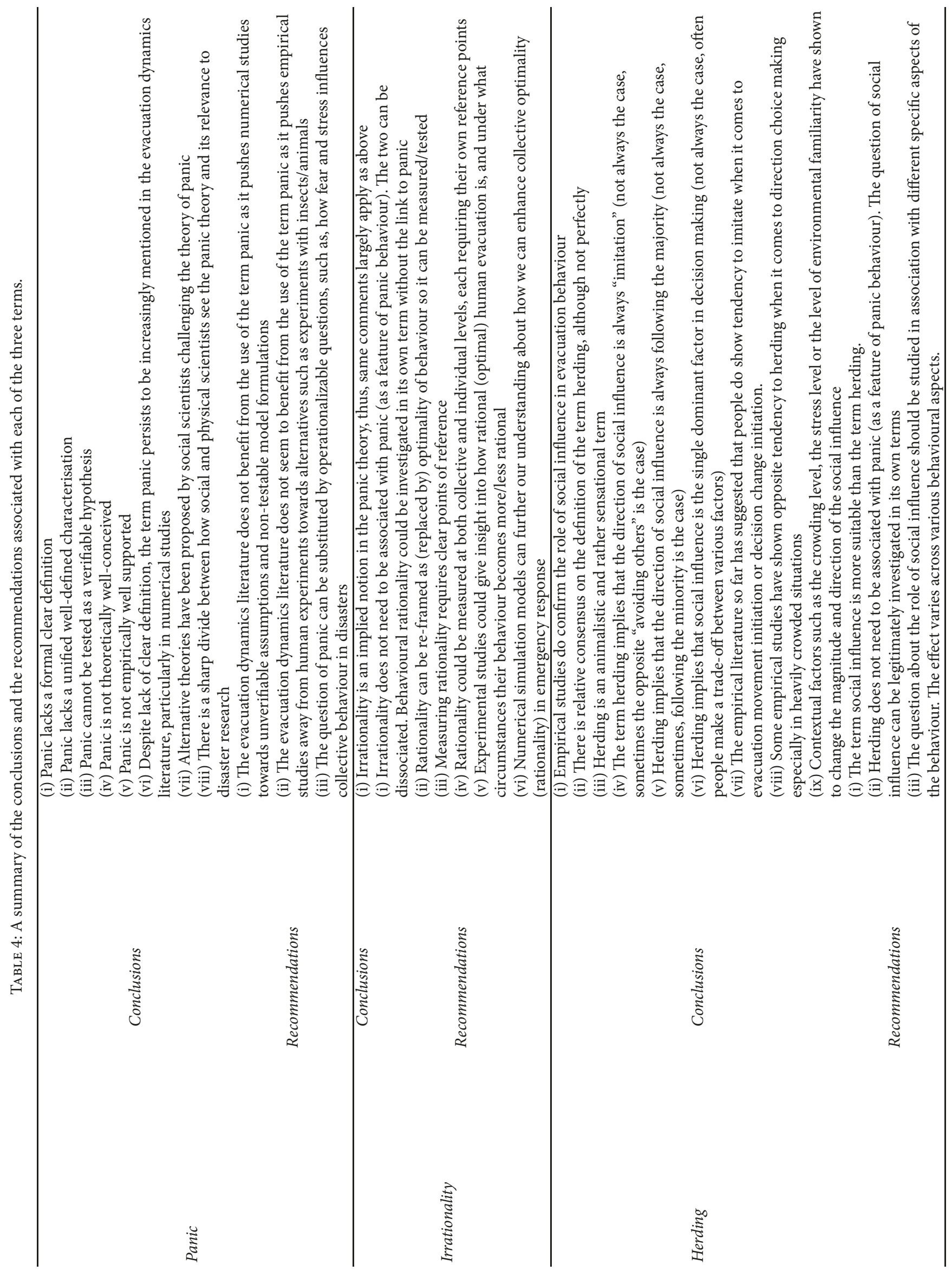




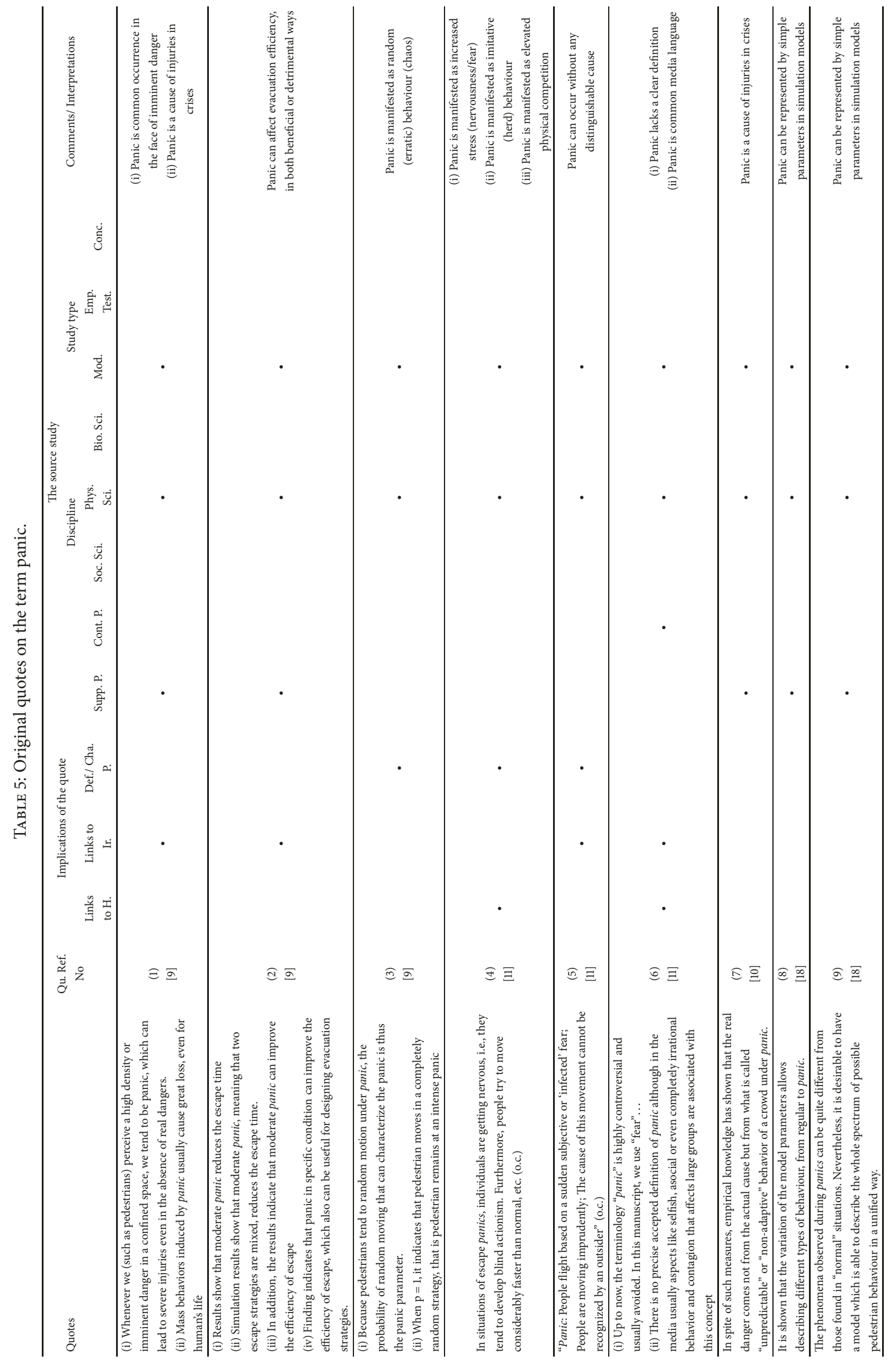




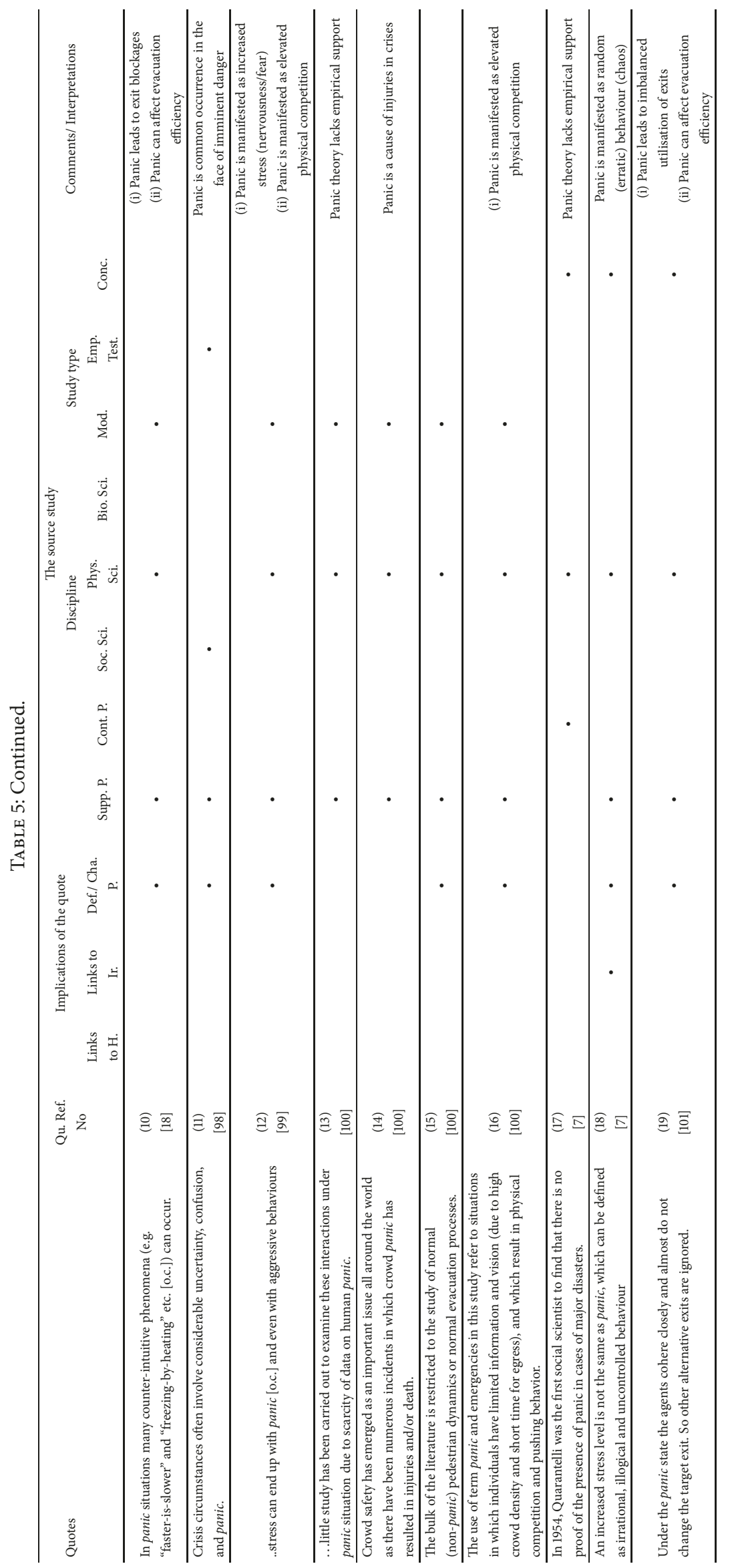




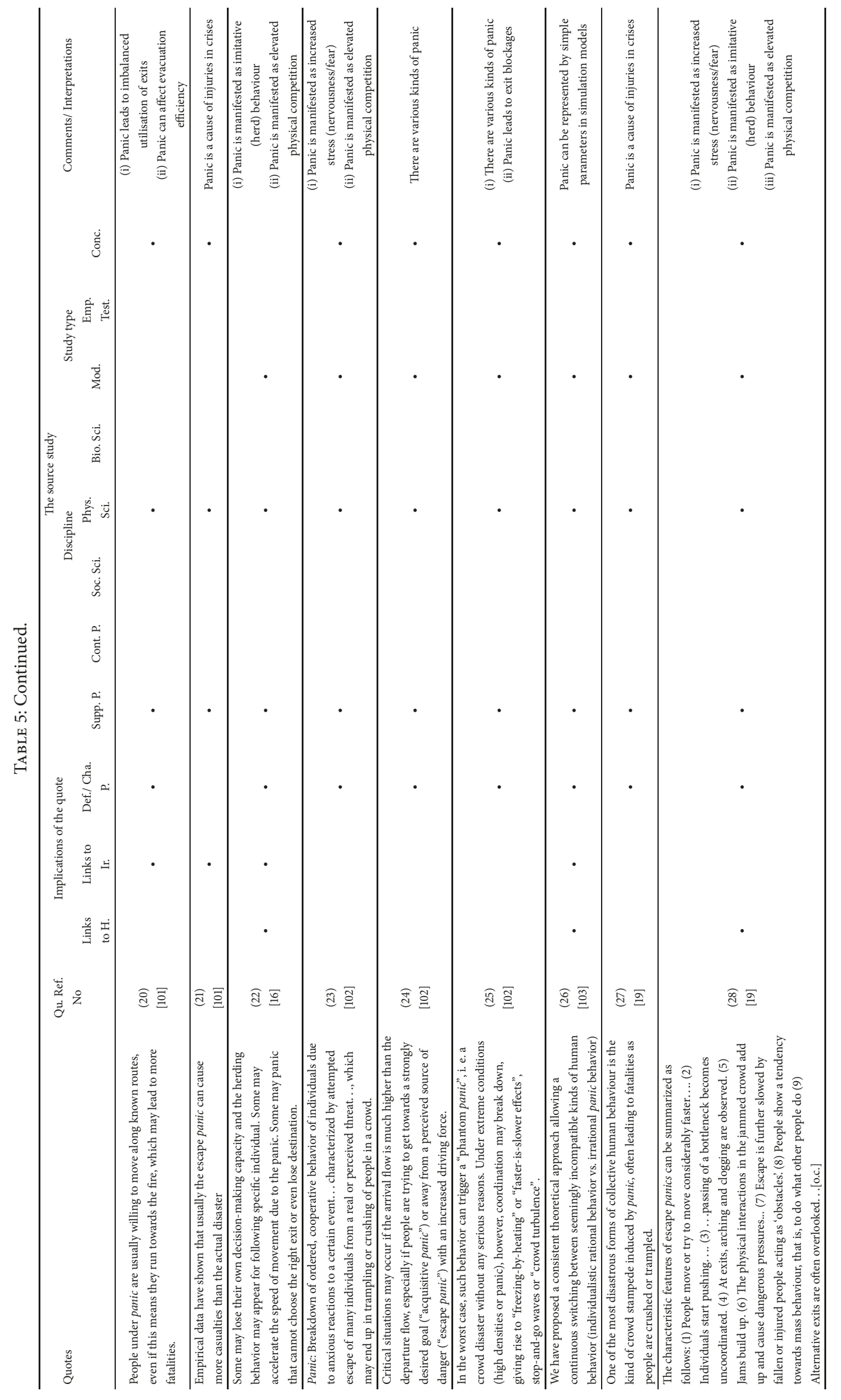




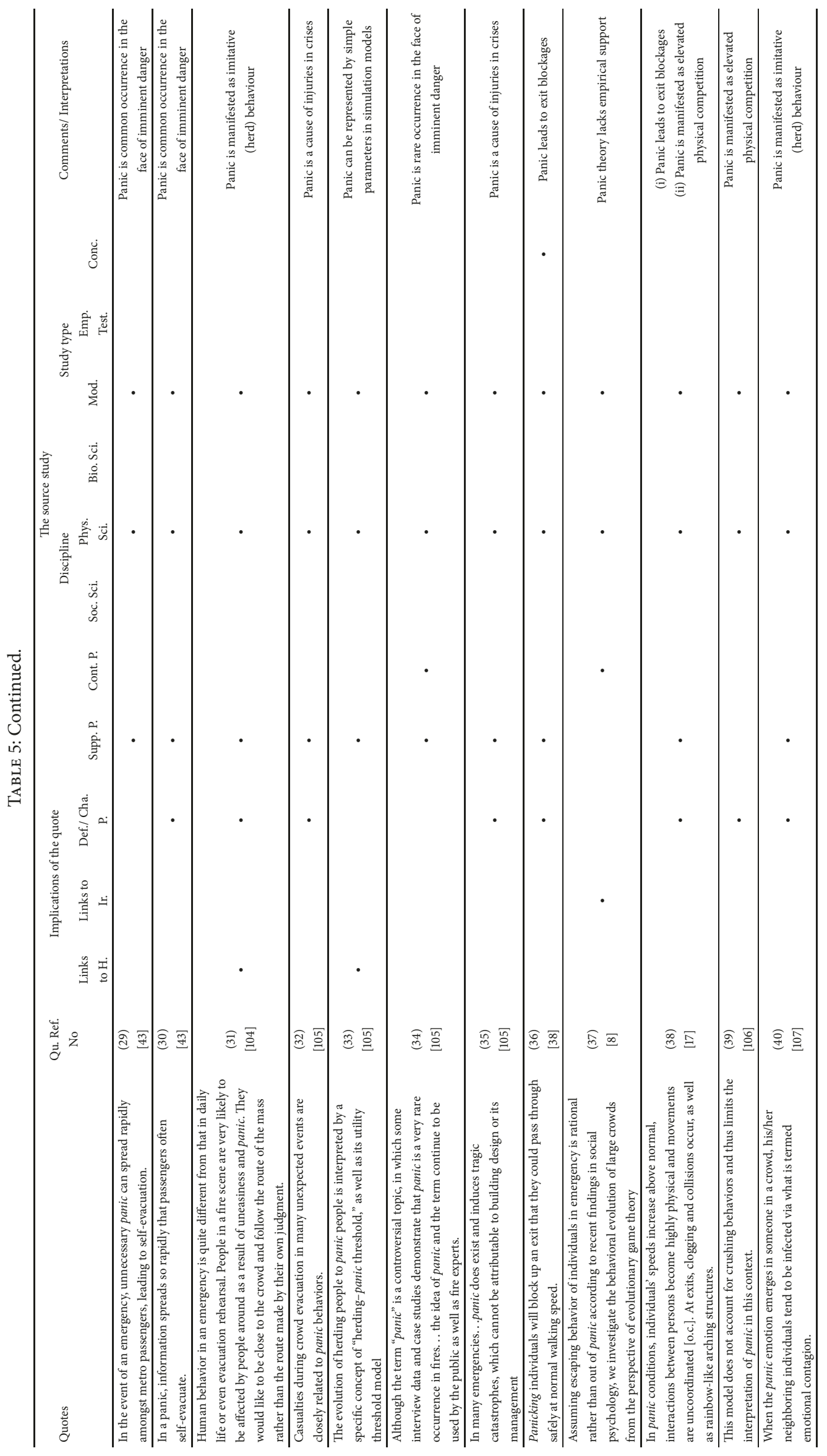




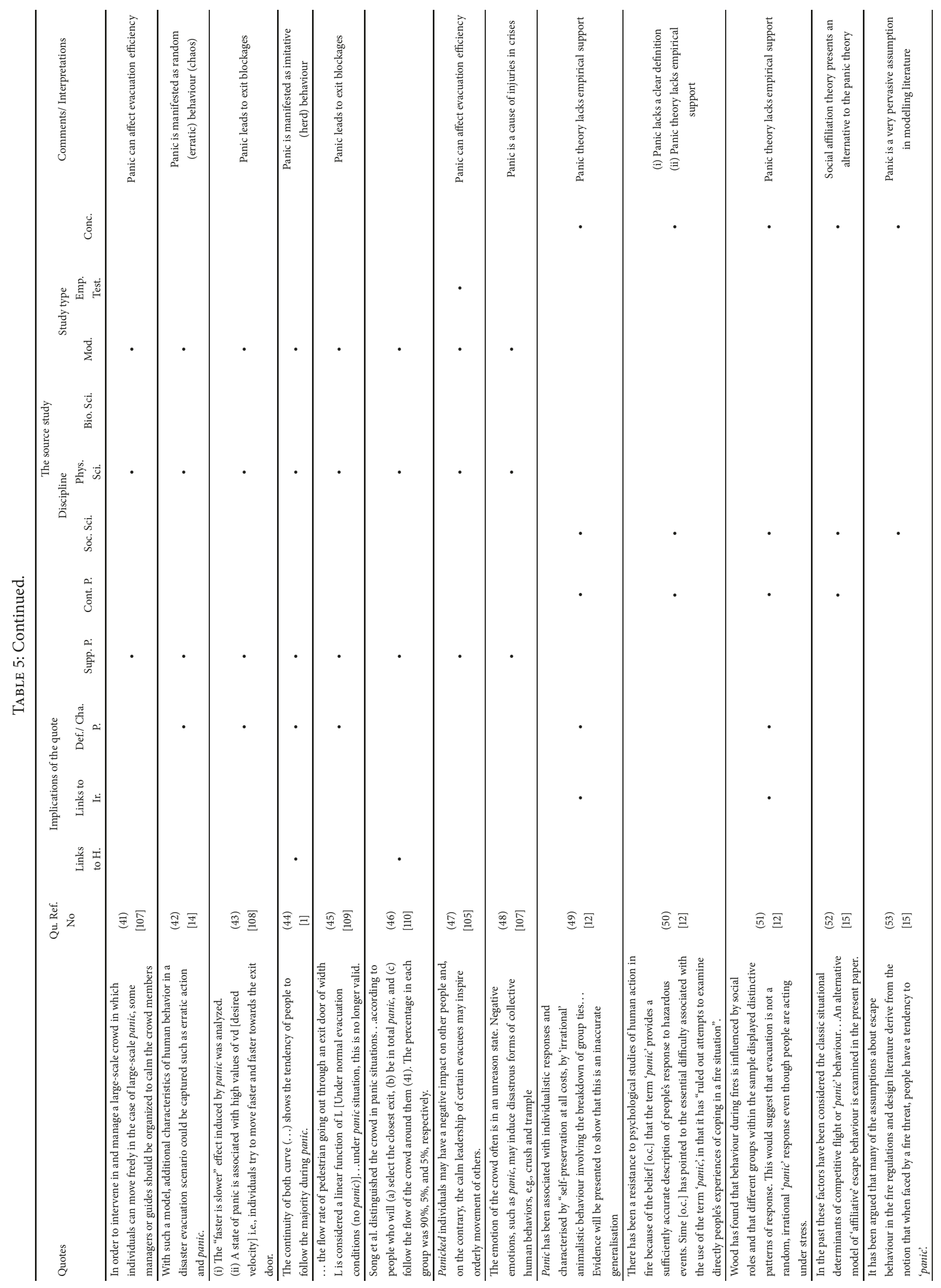




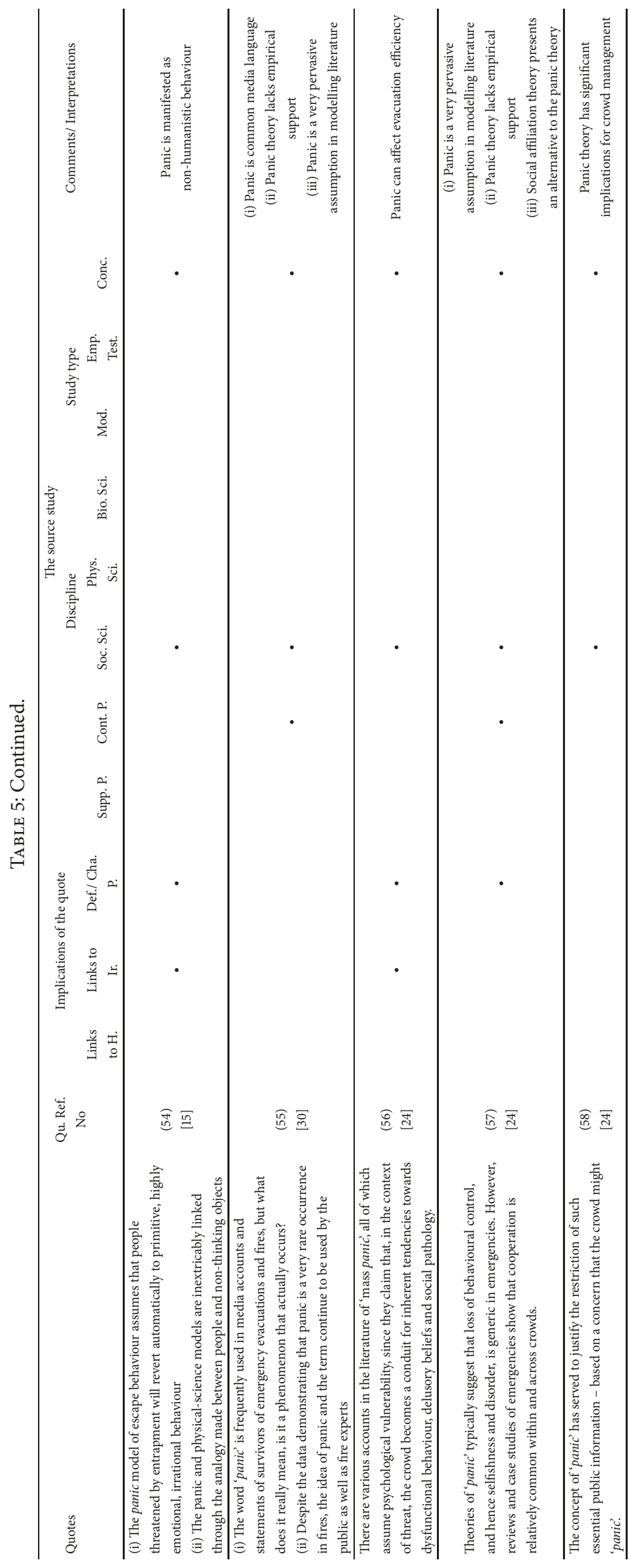




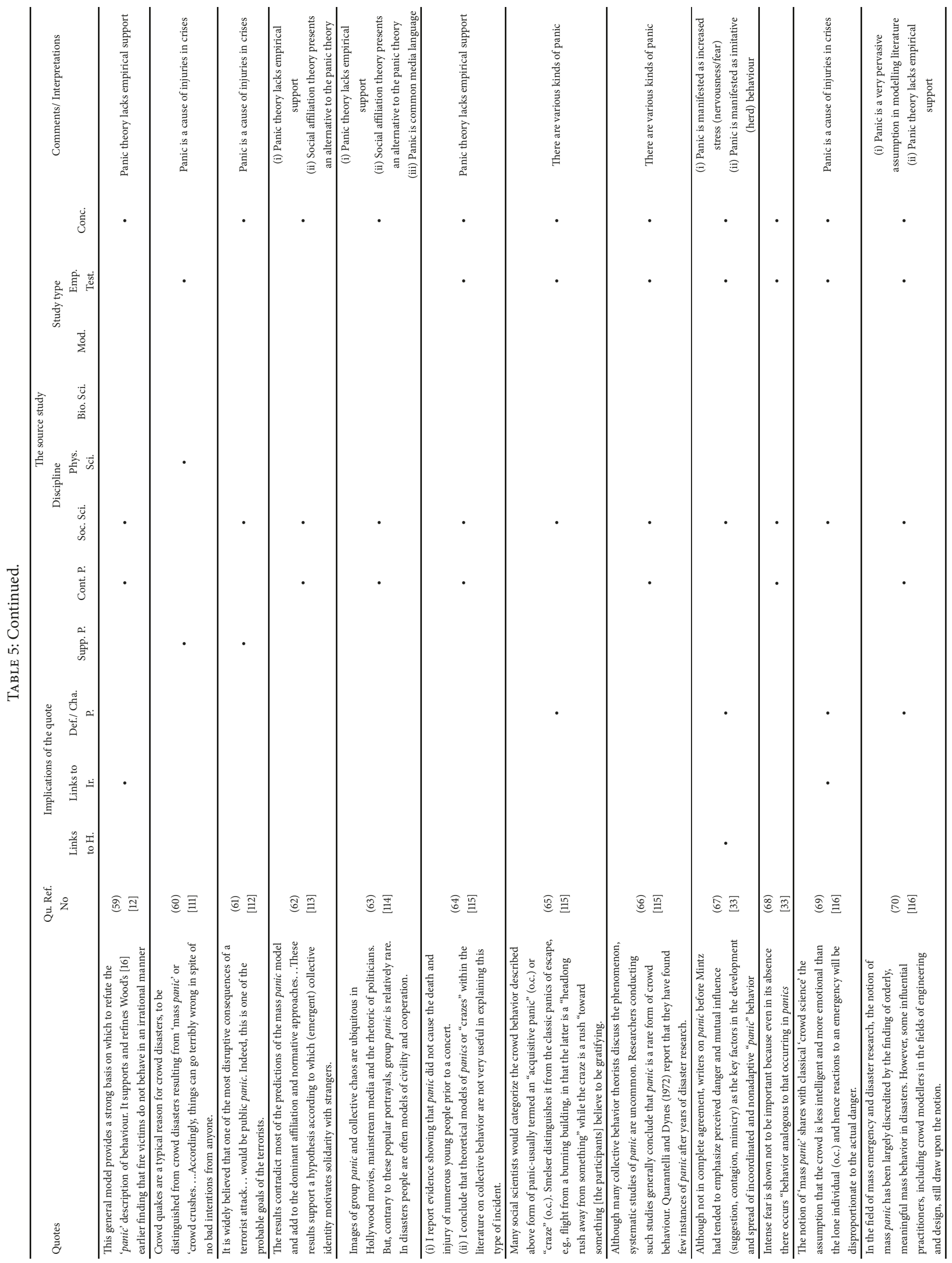




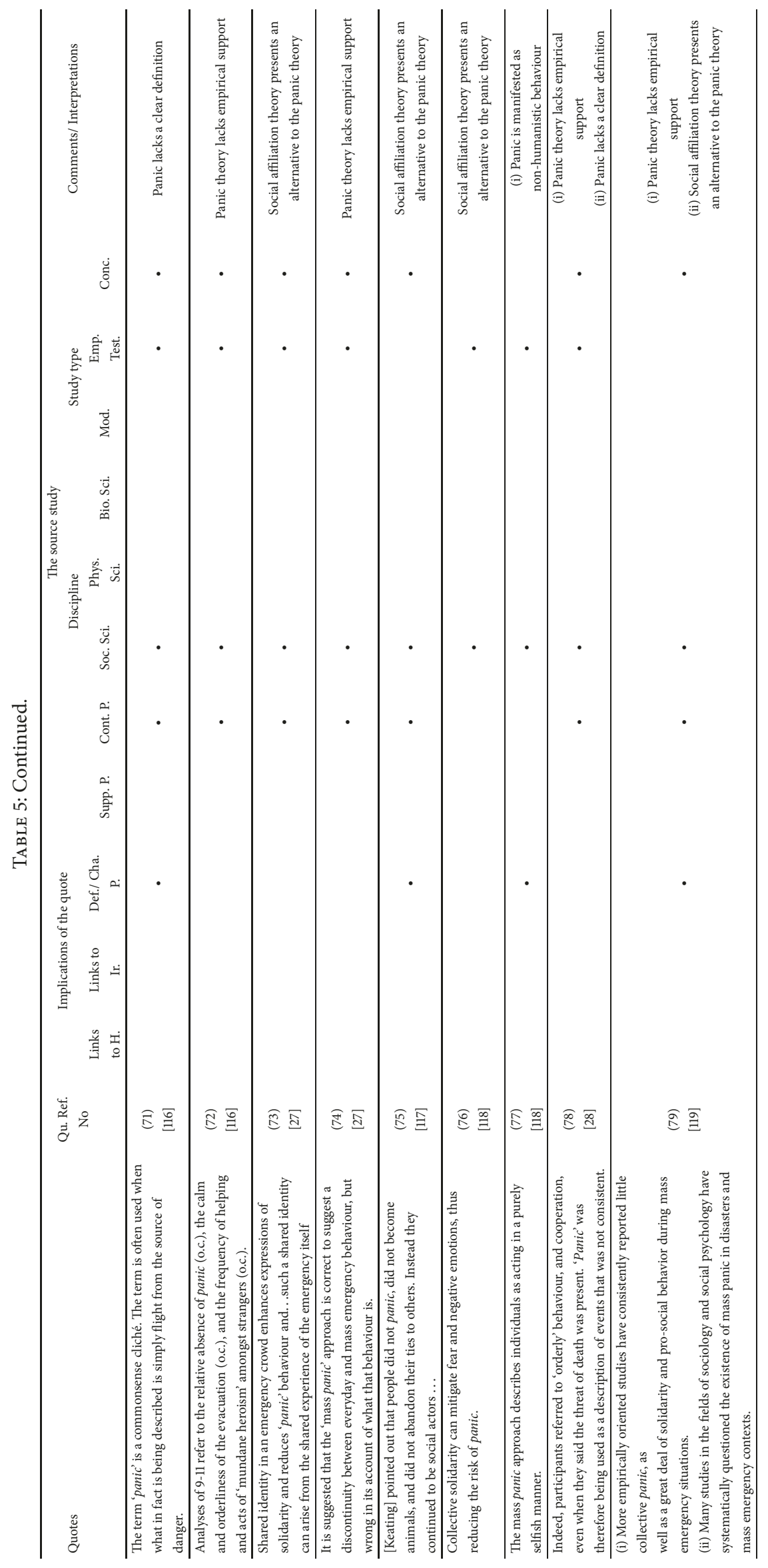




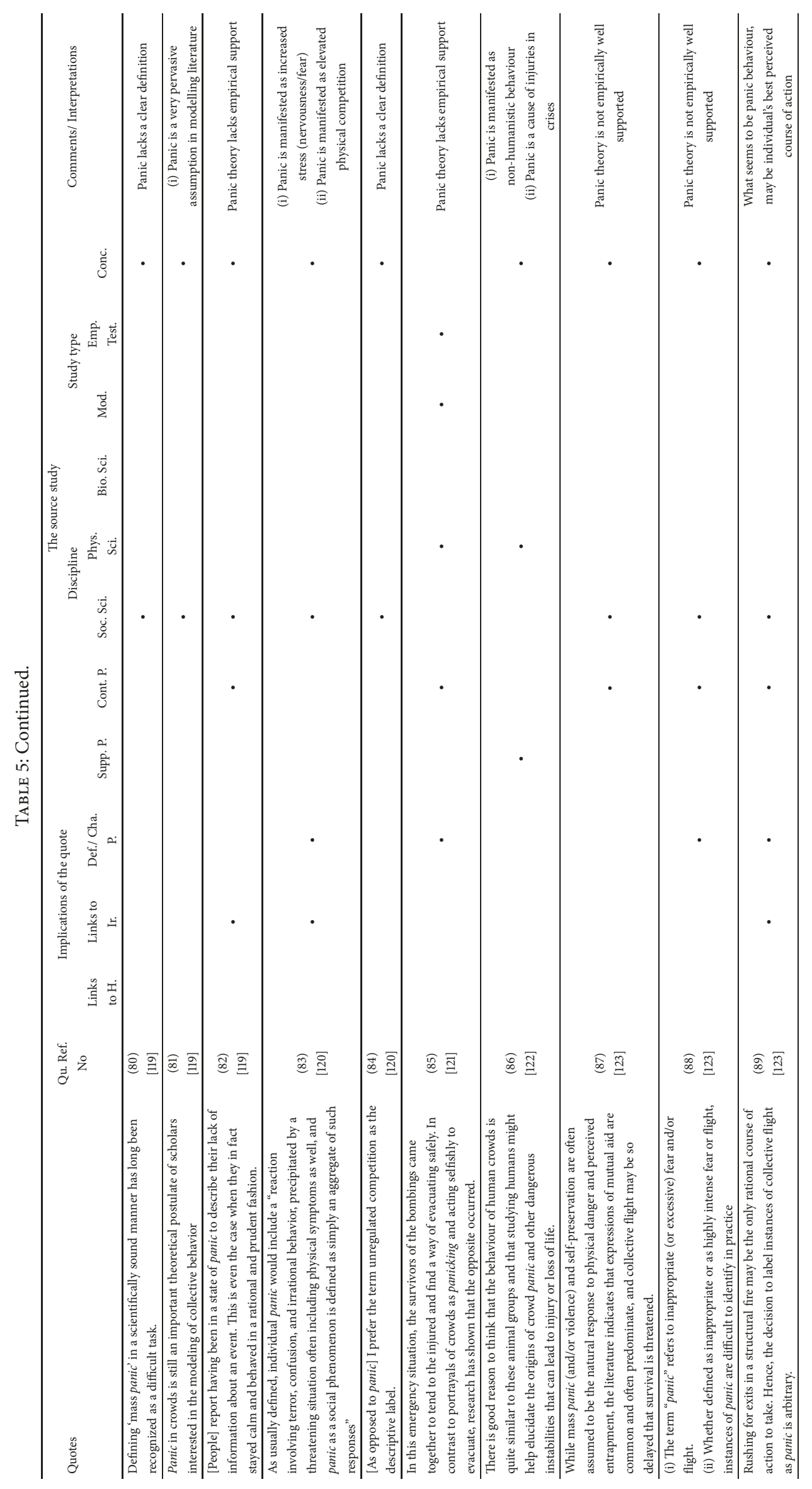




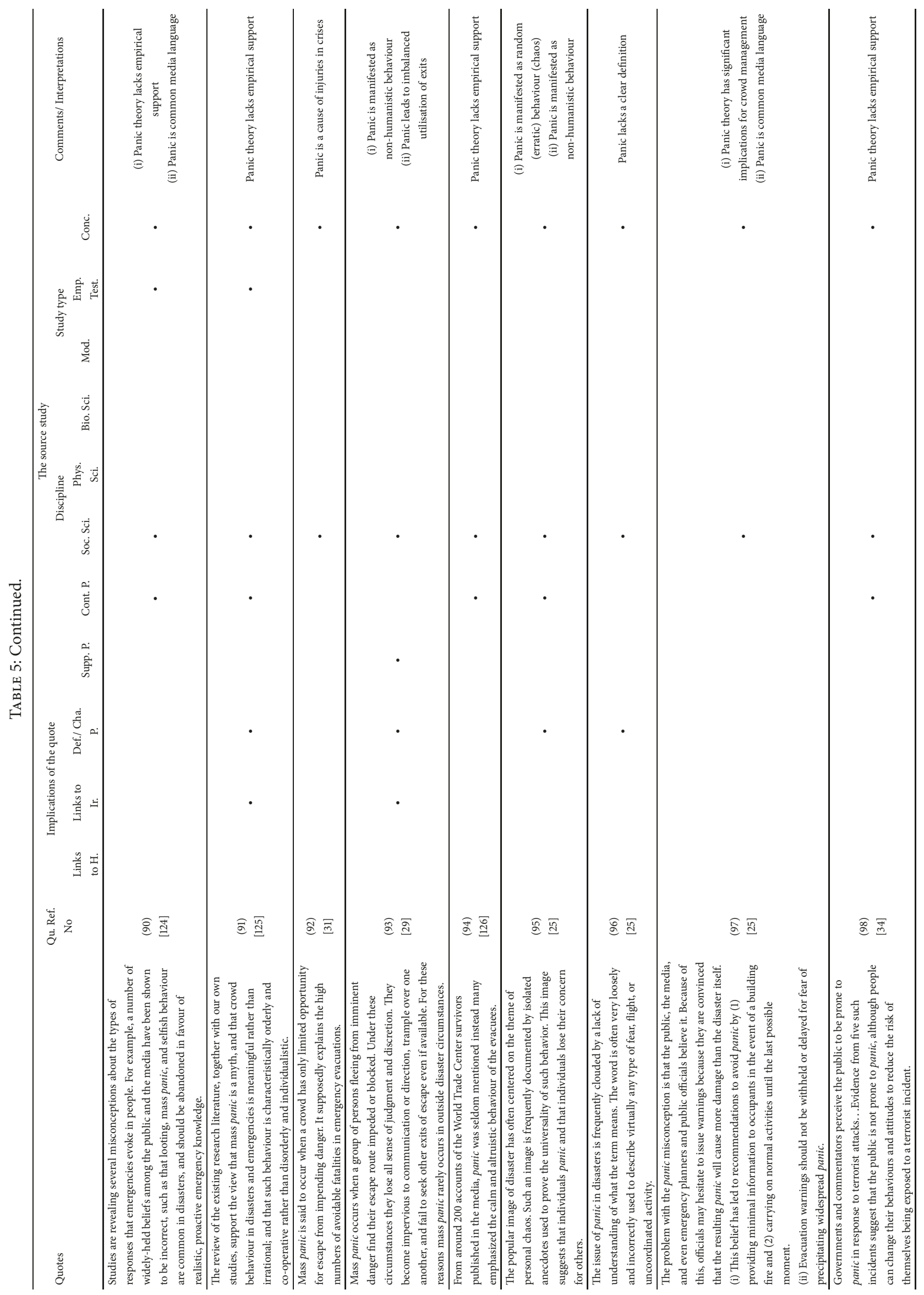




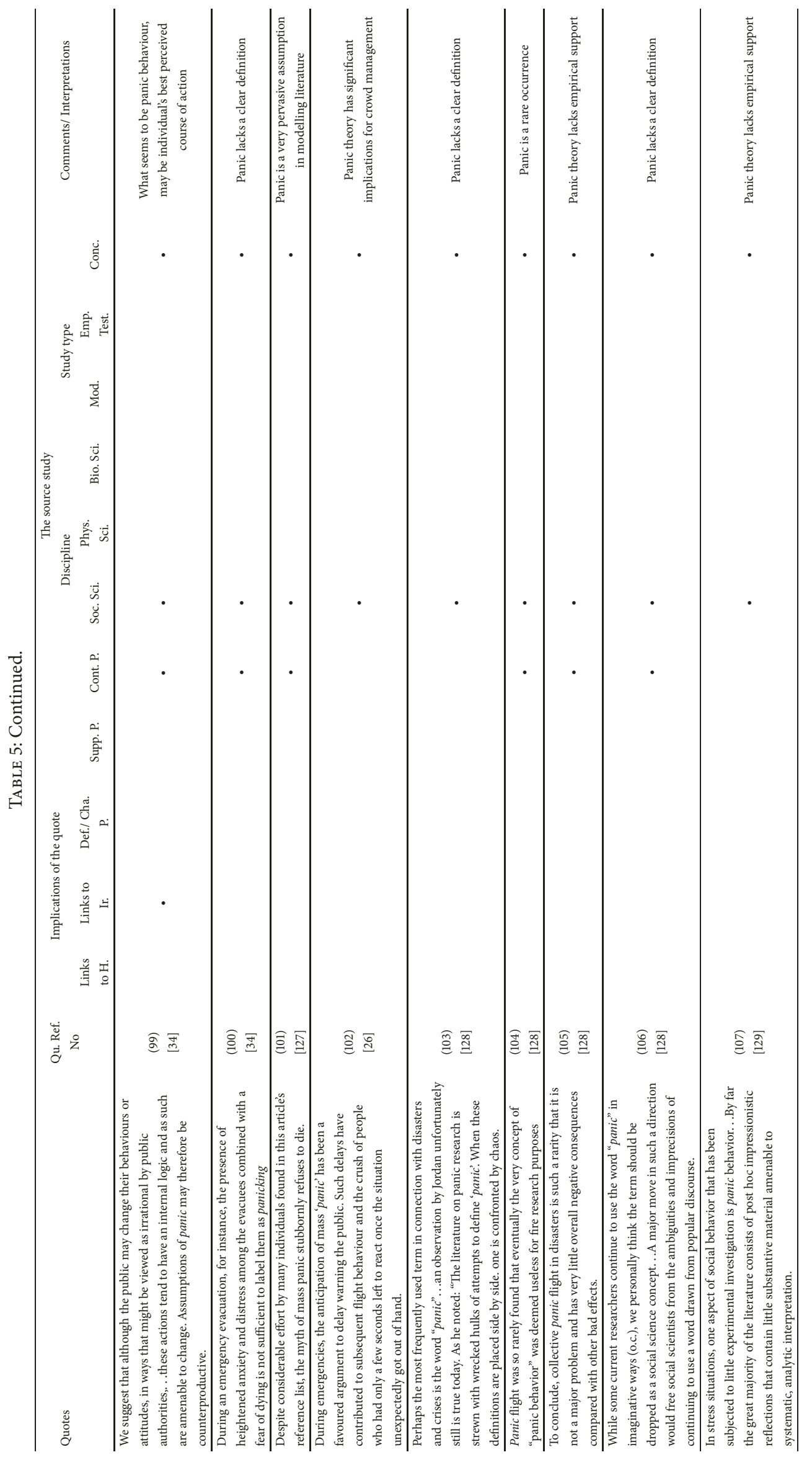




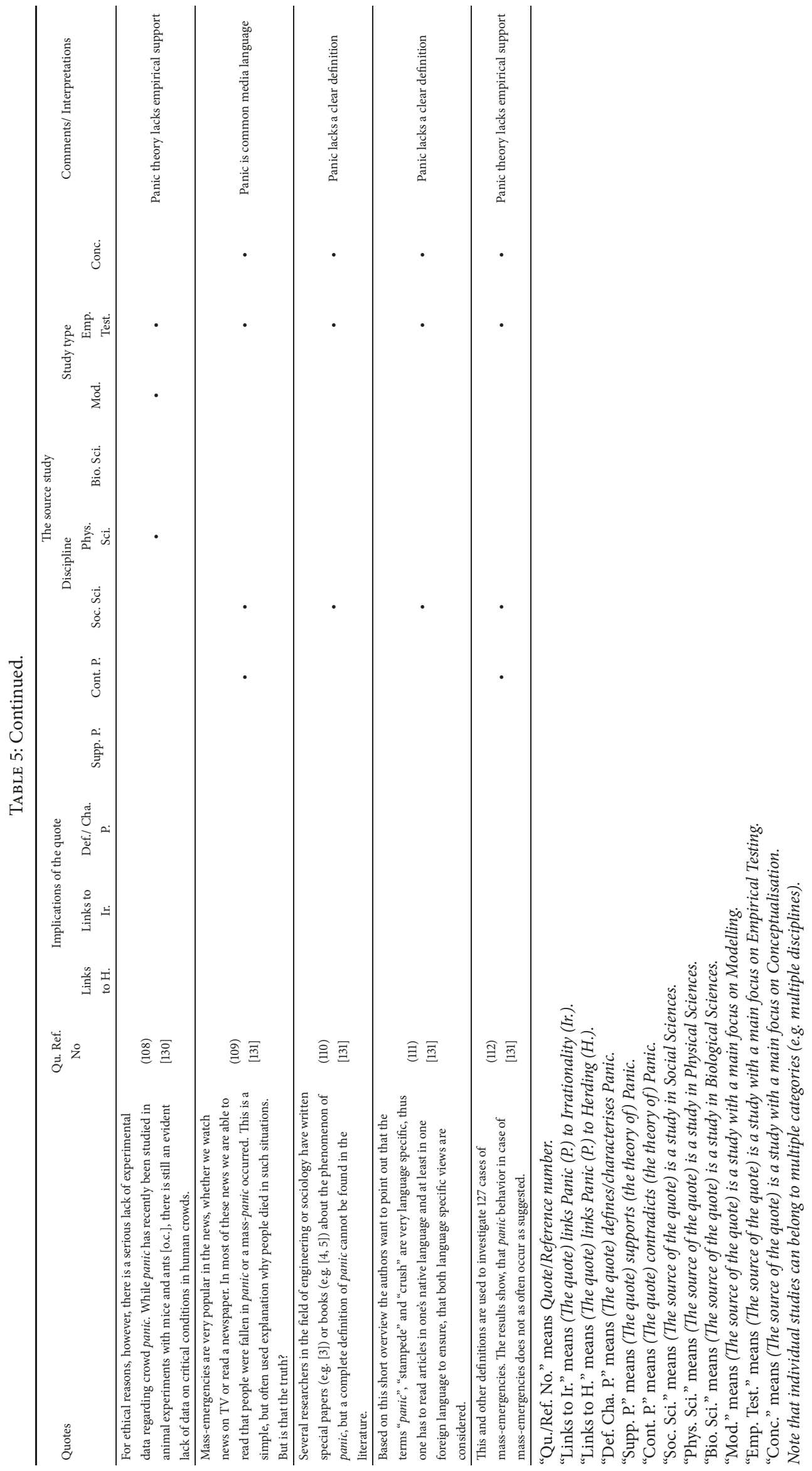




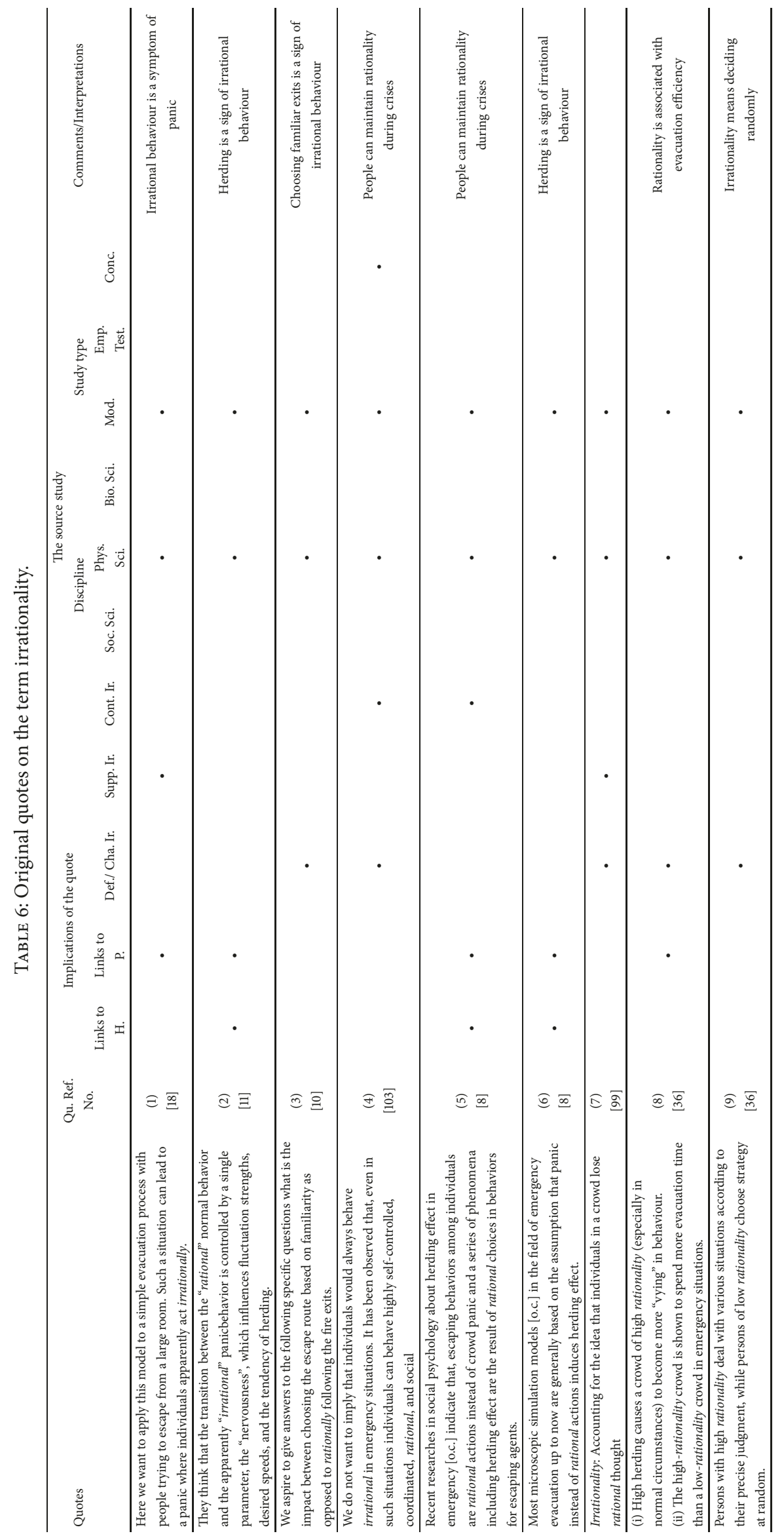




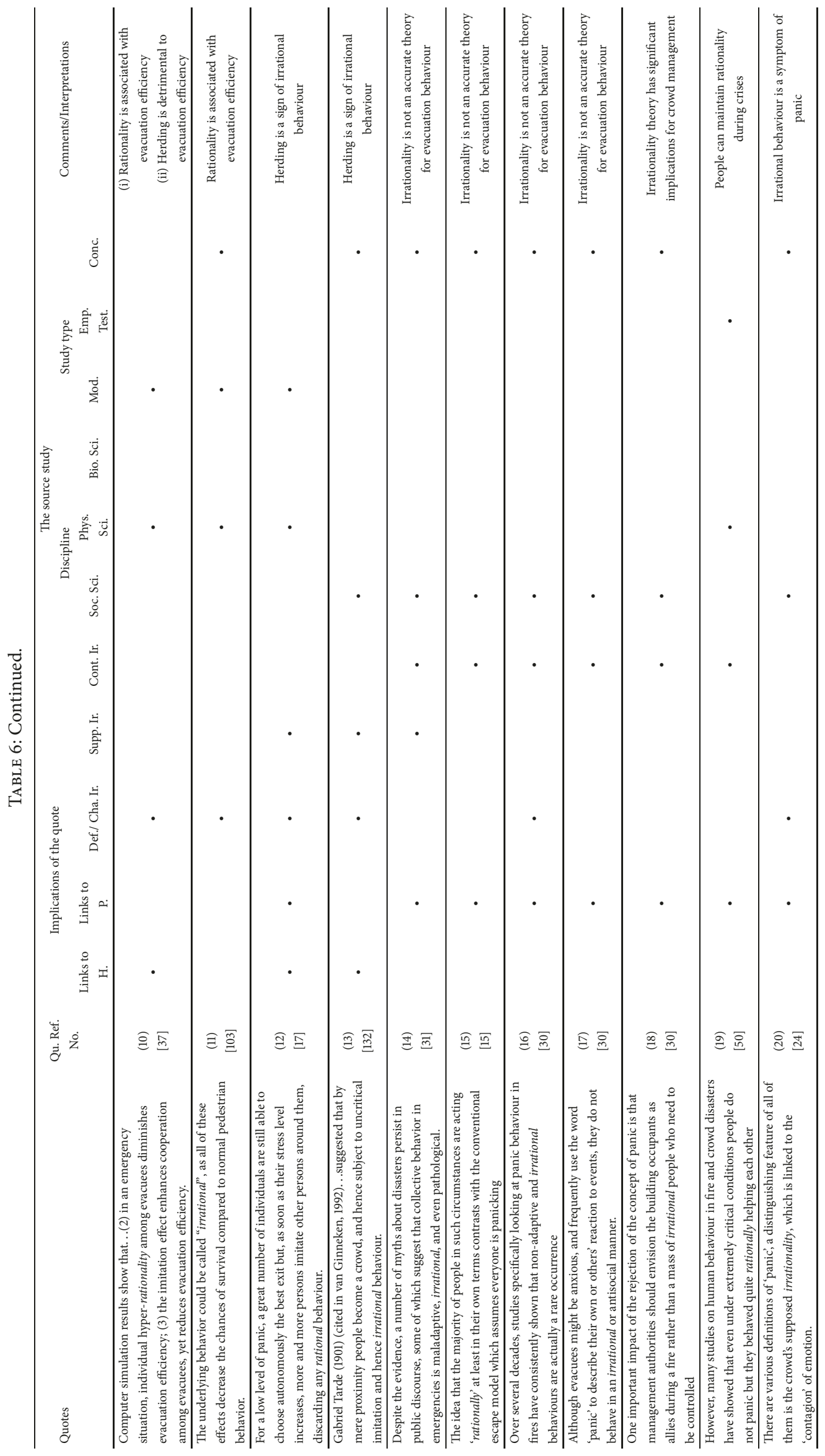




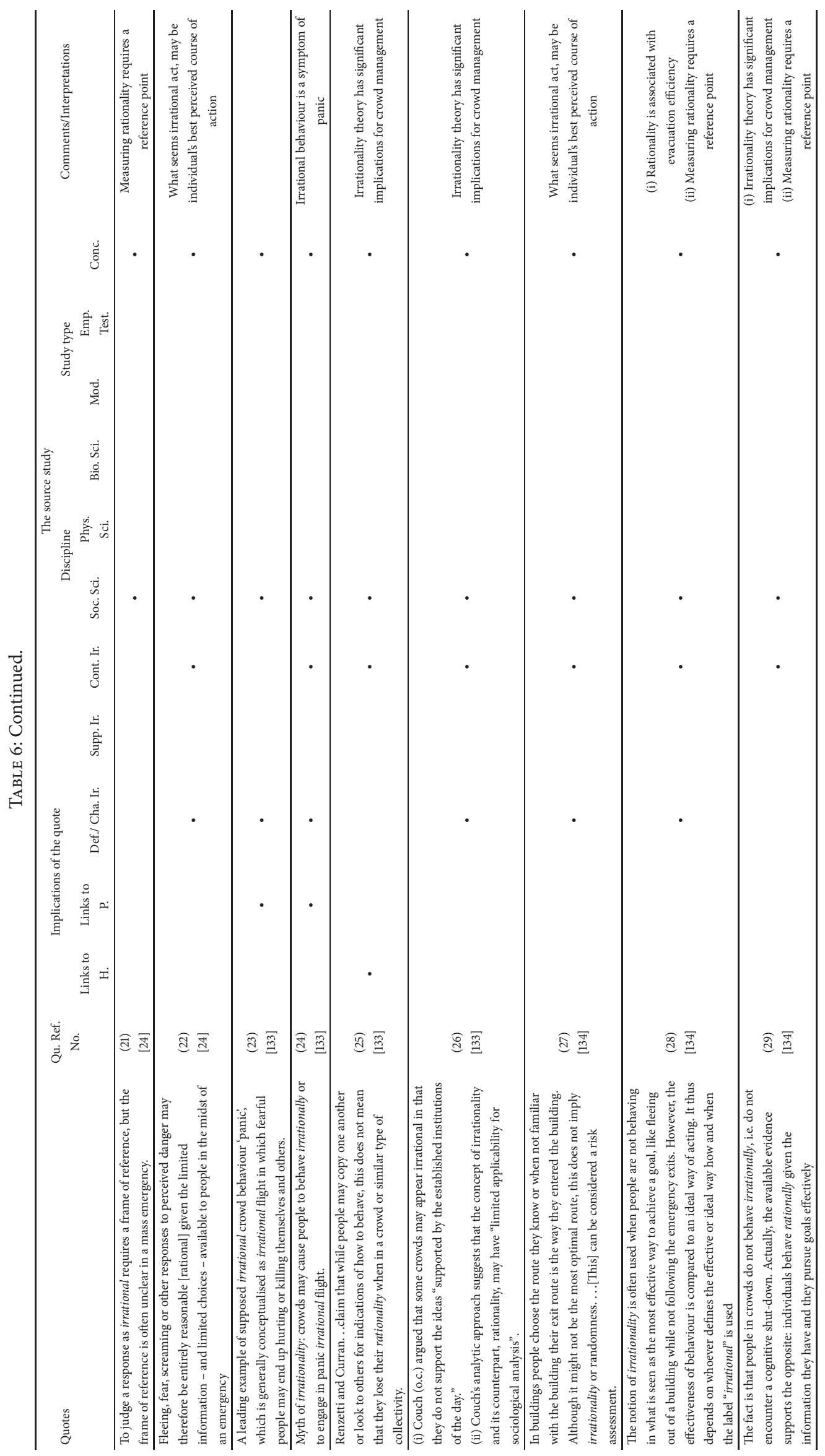




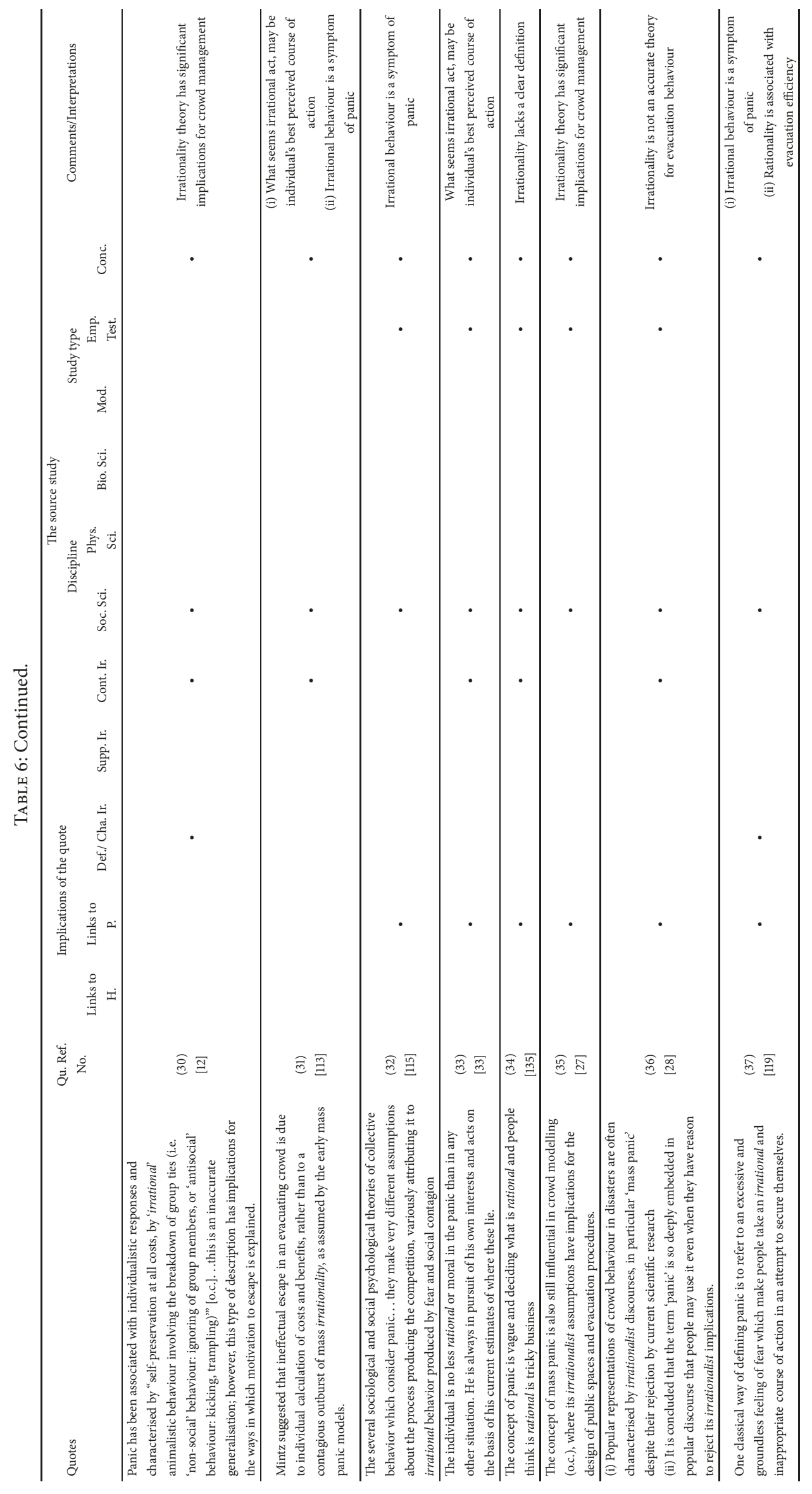




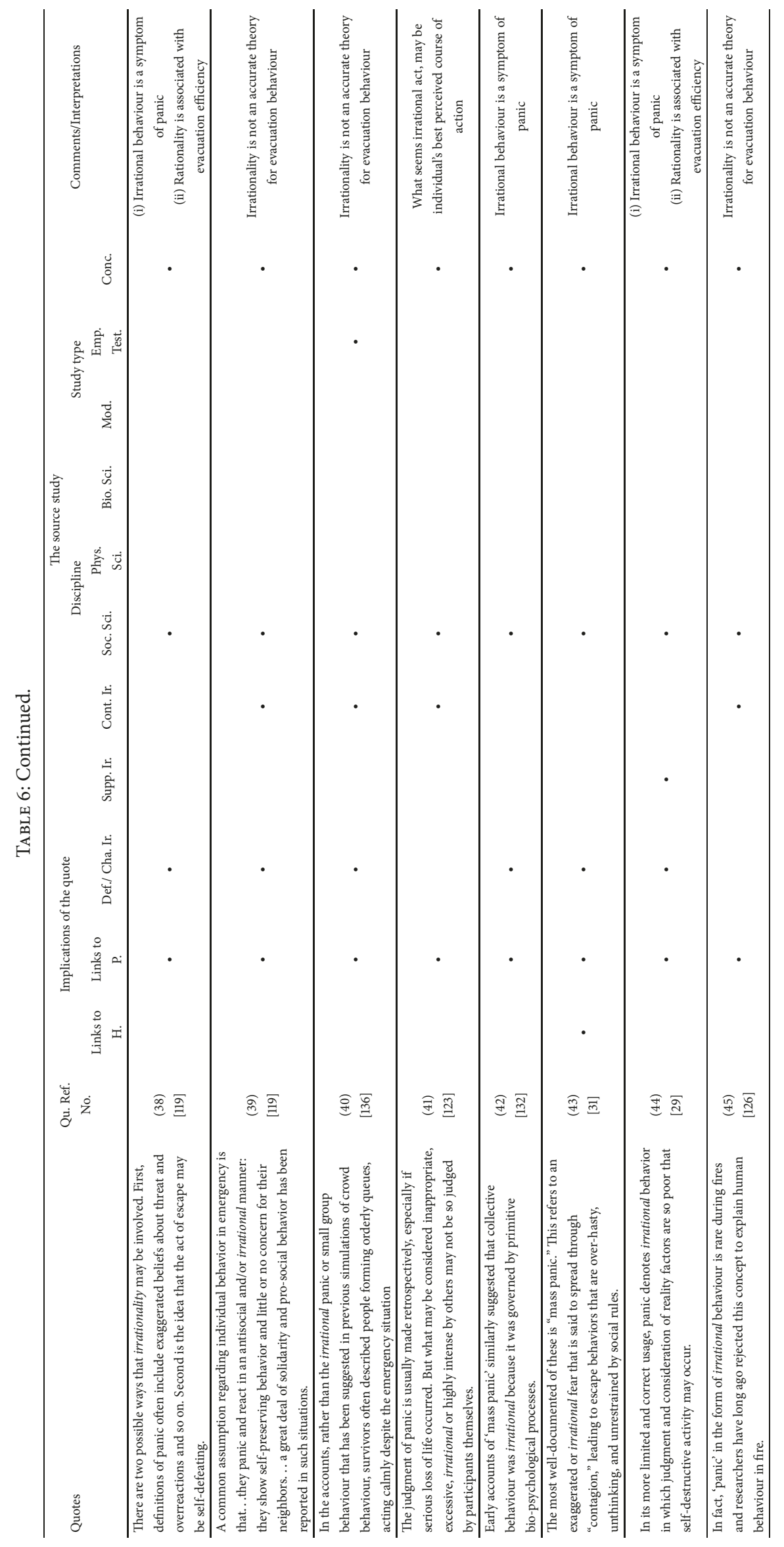




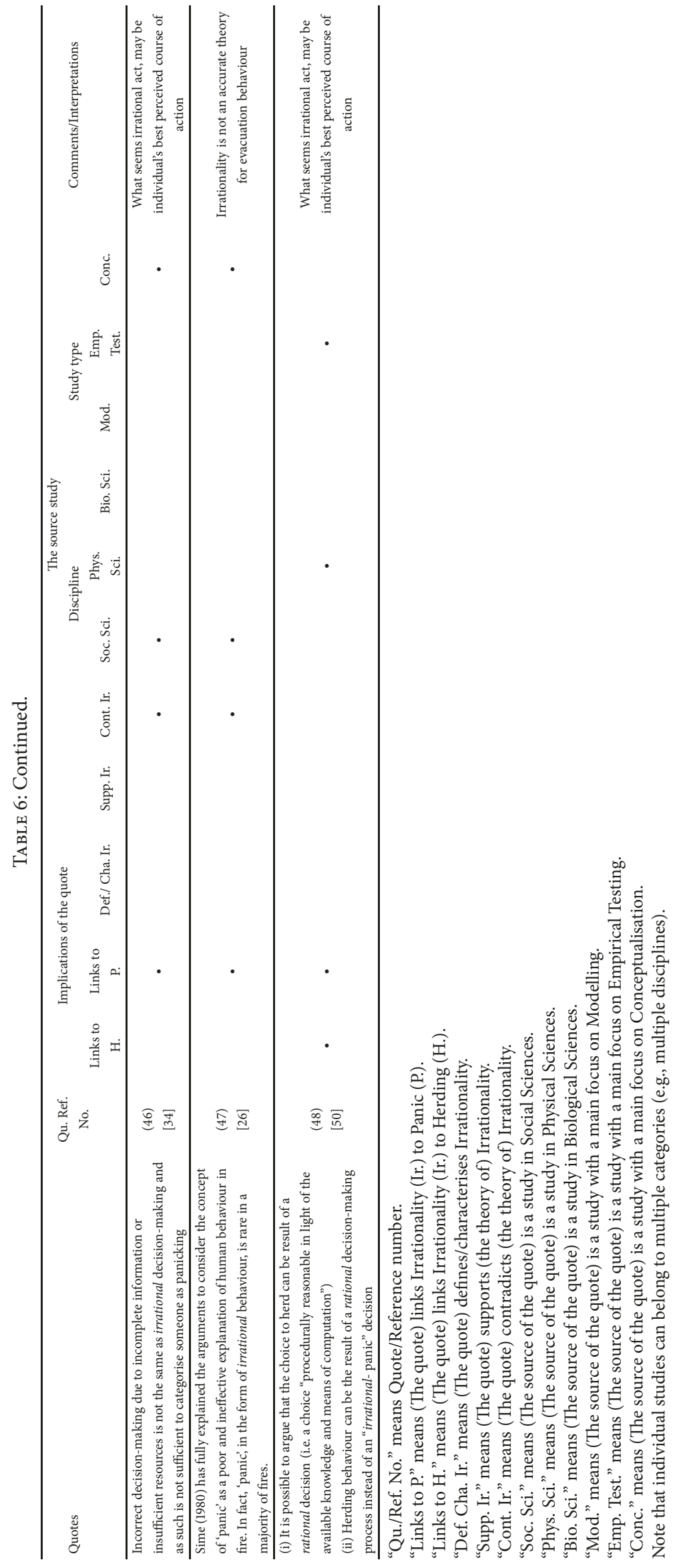




$$
1
$$




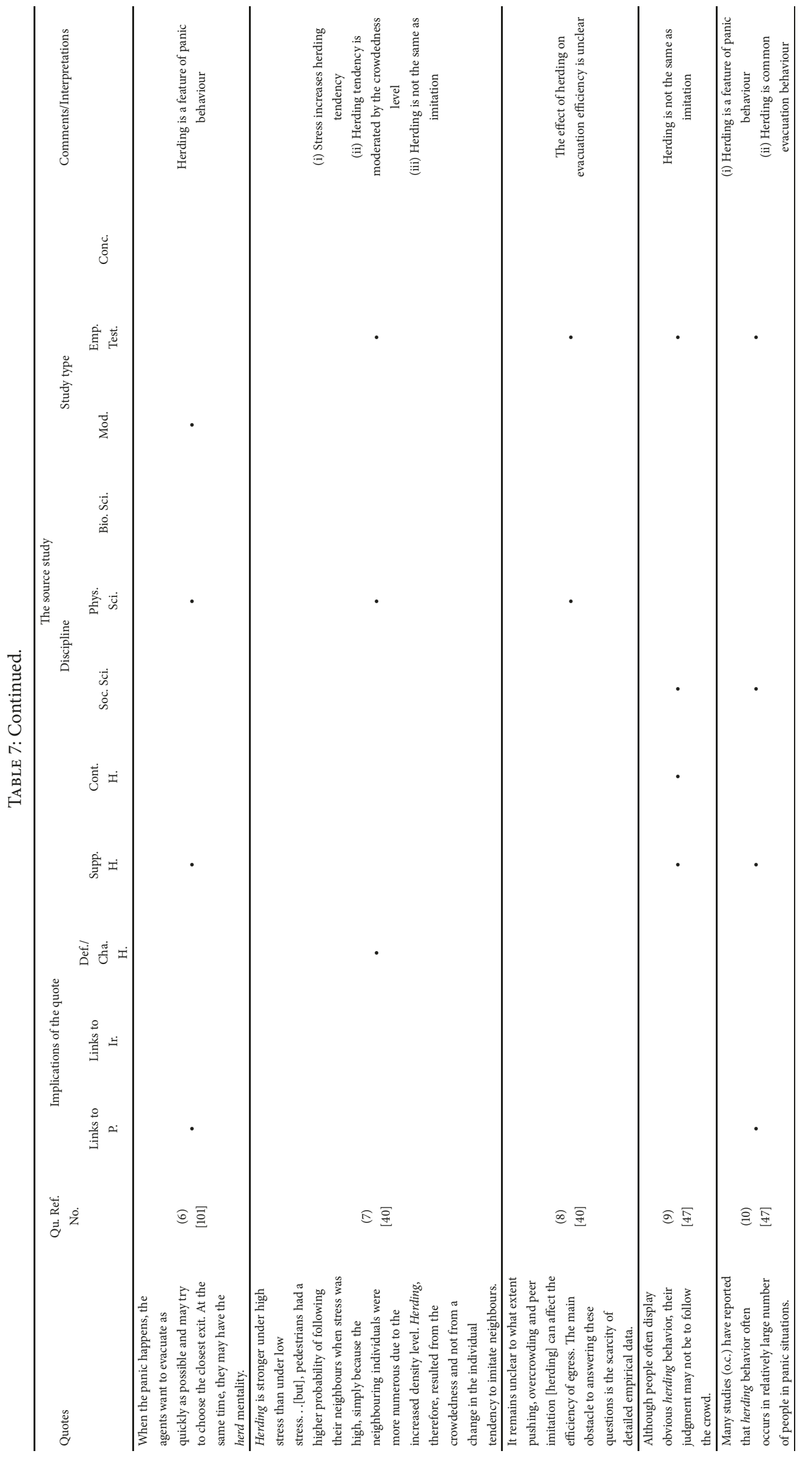




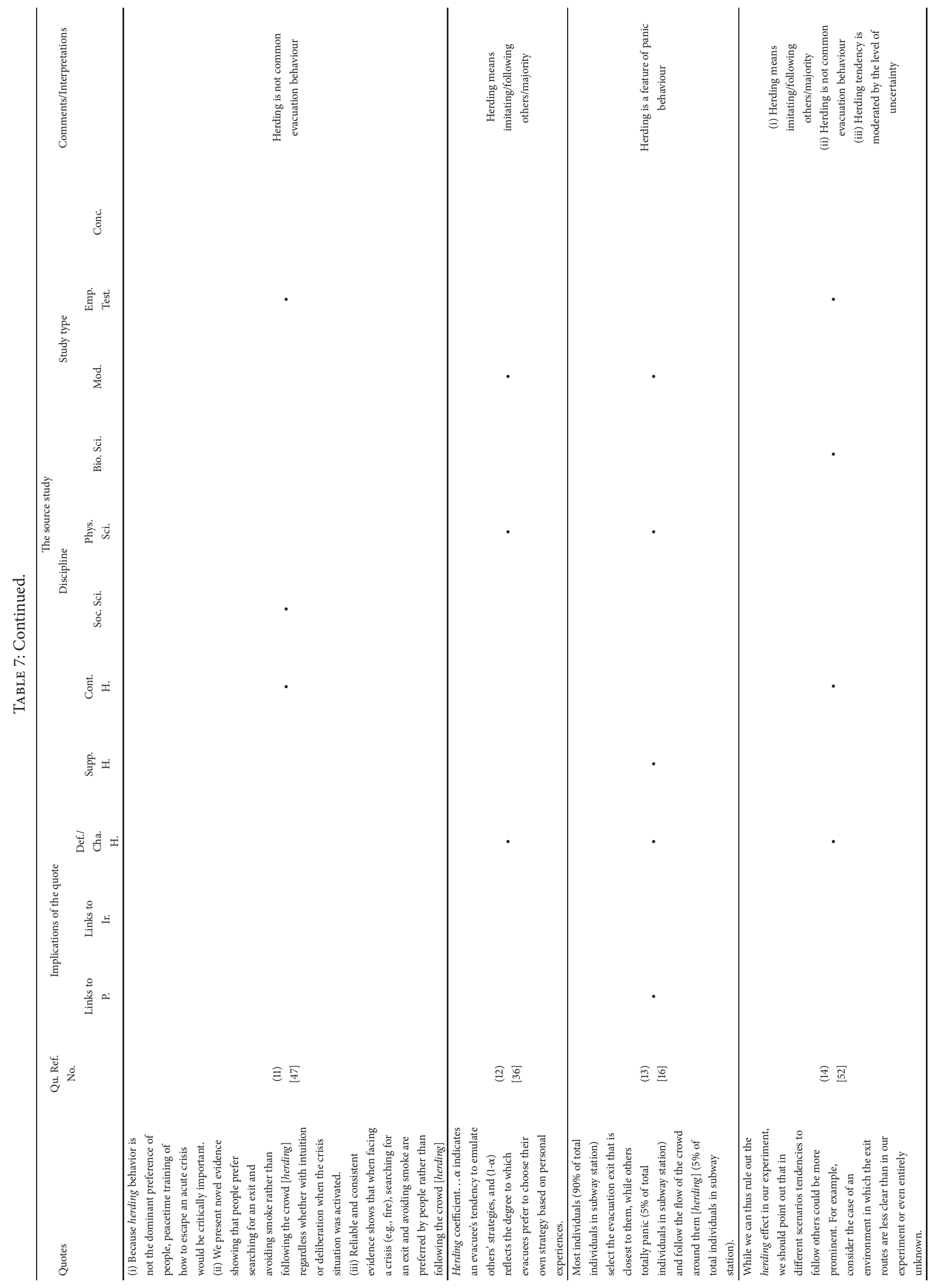




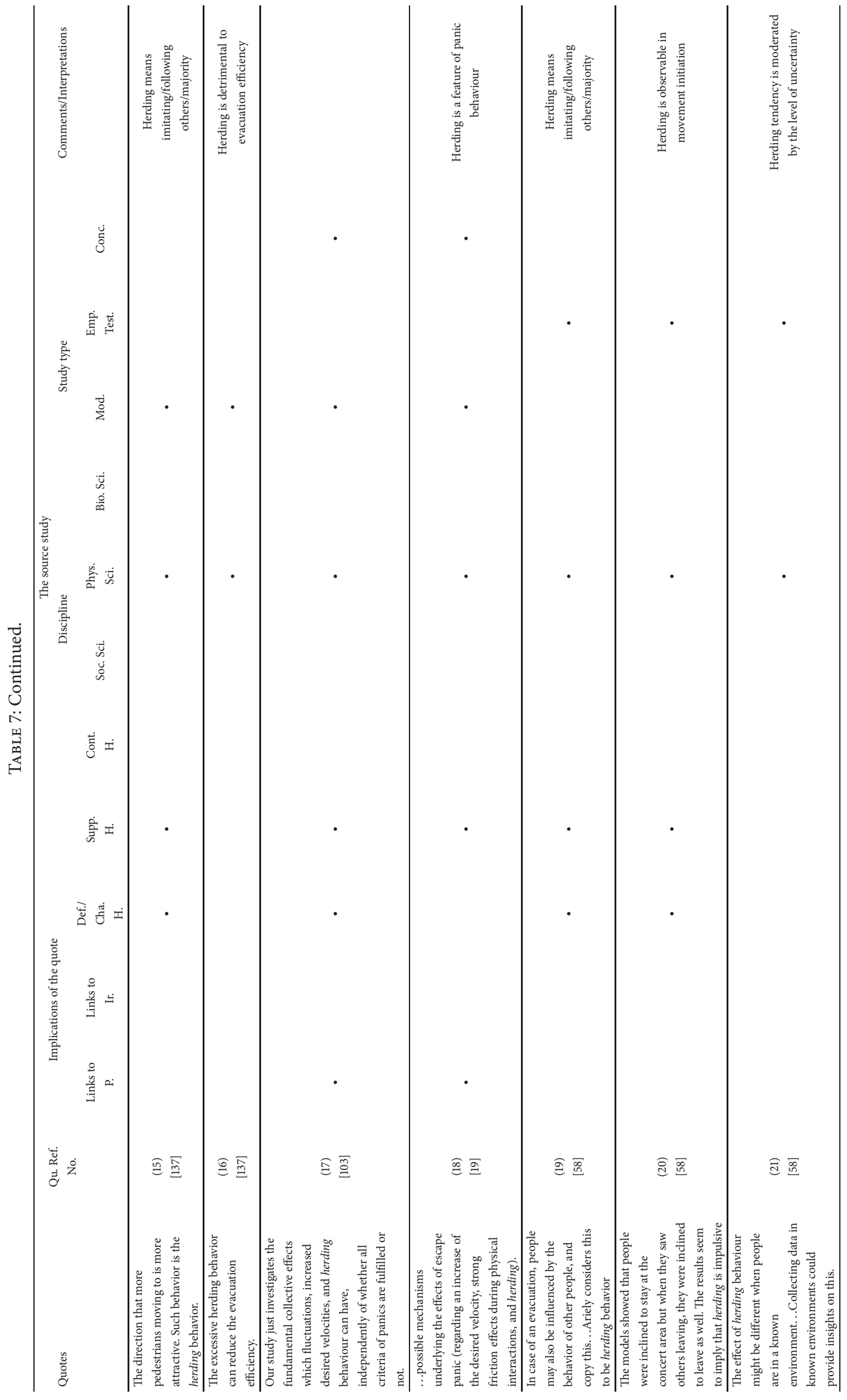




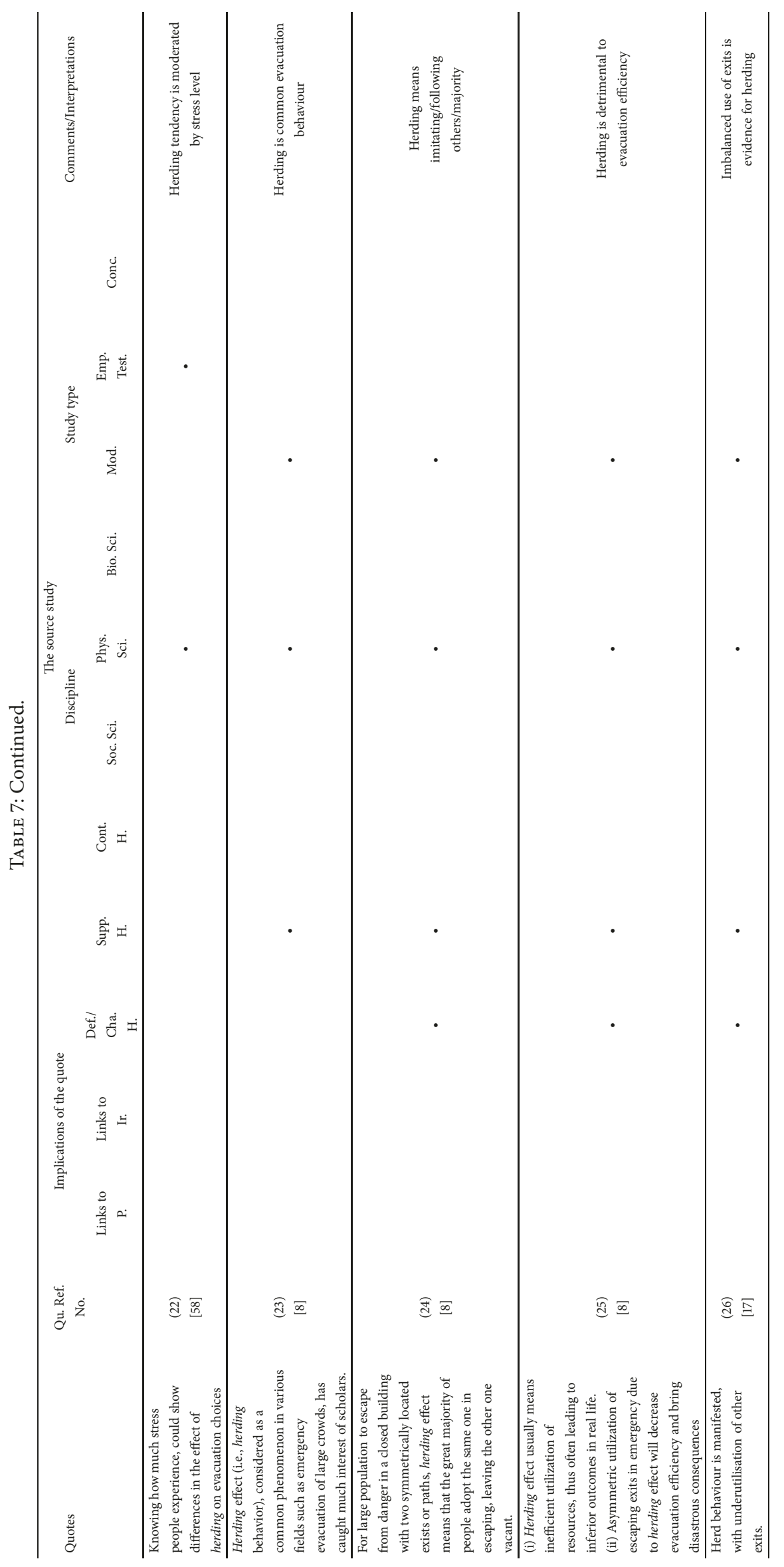




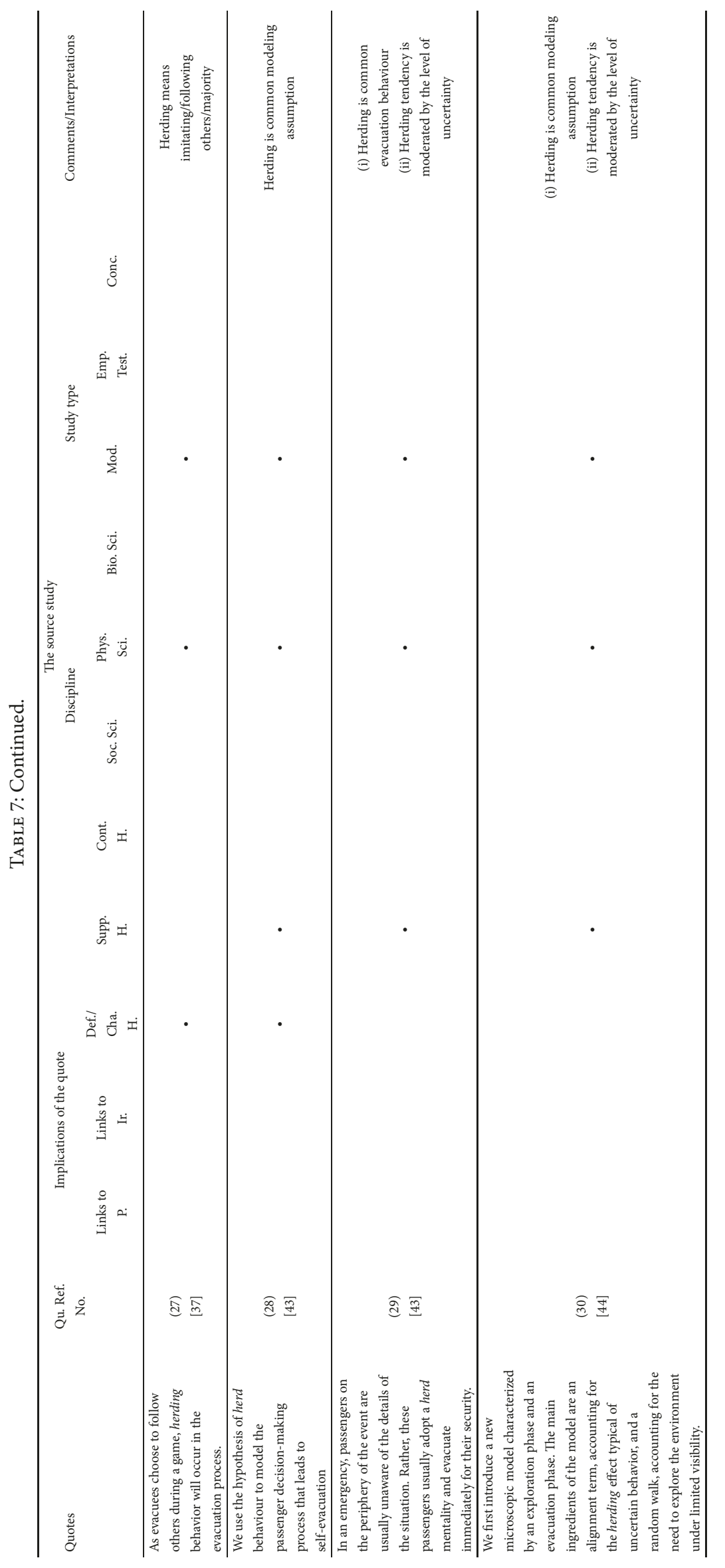




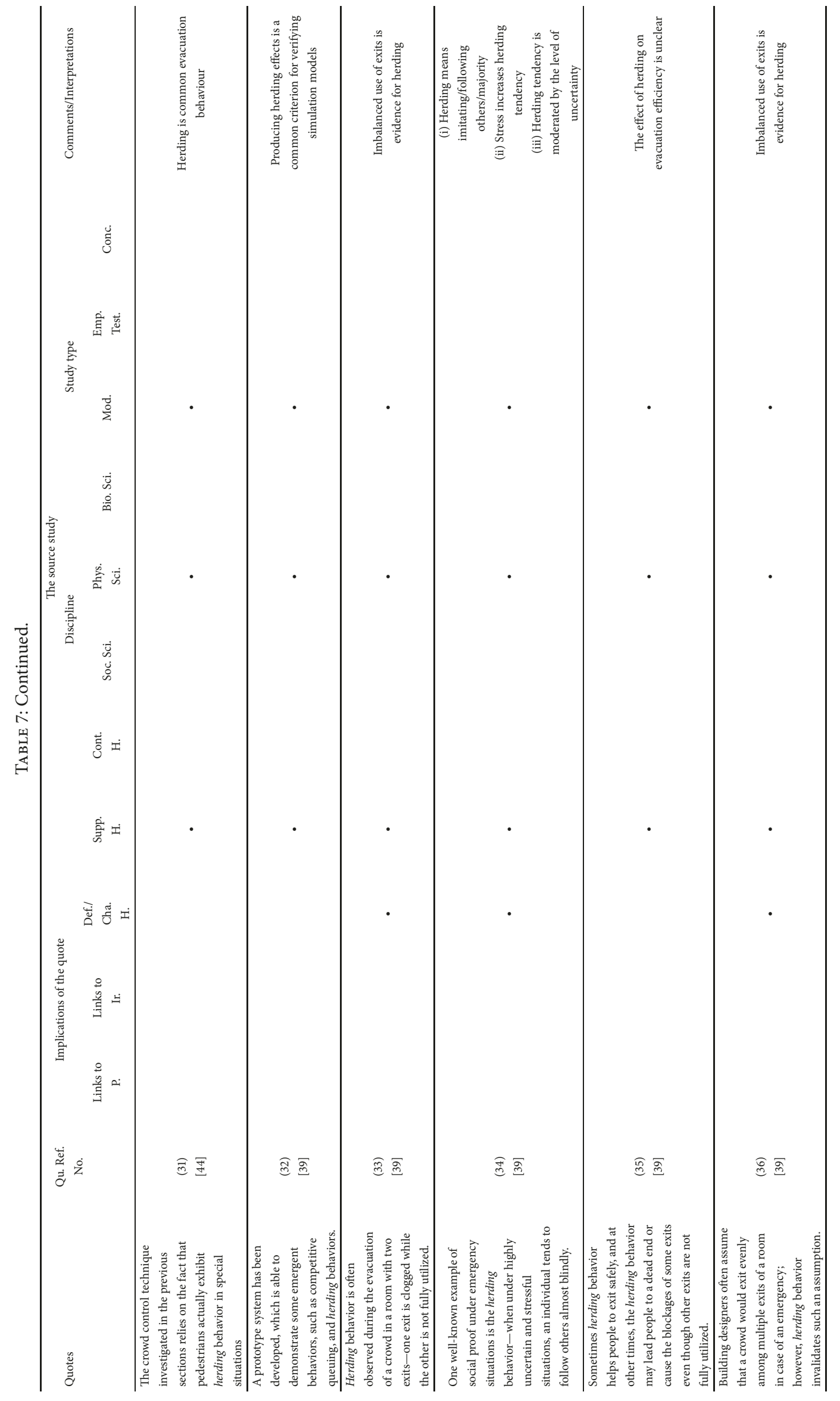




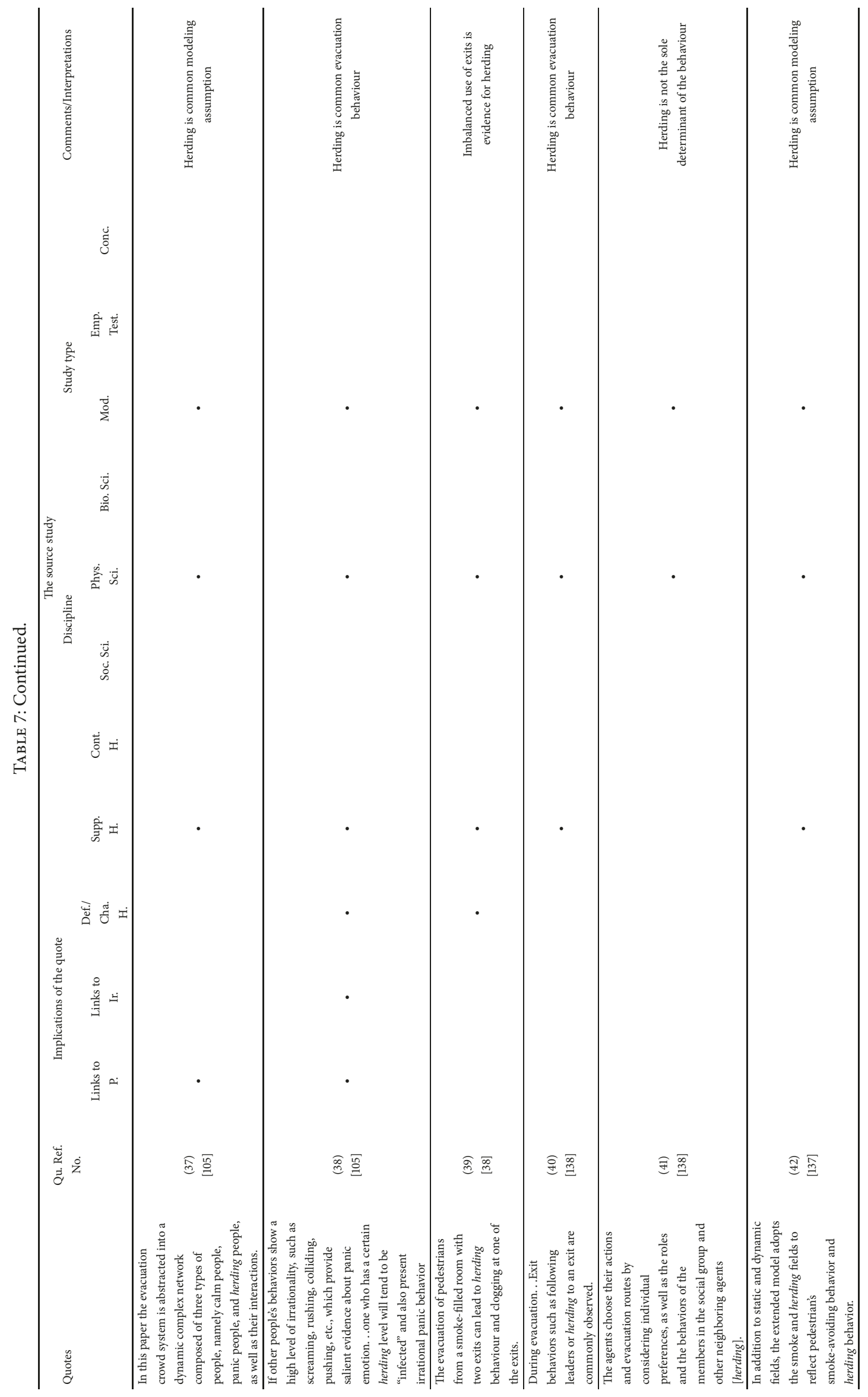




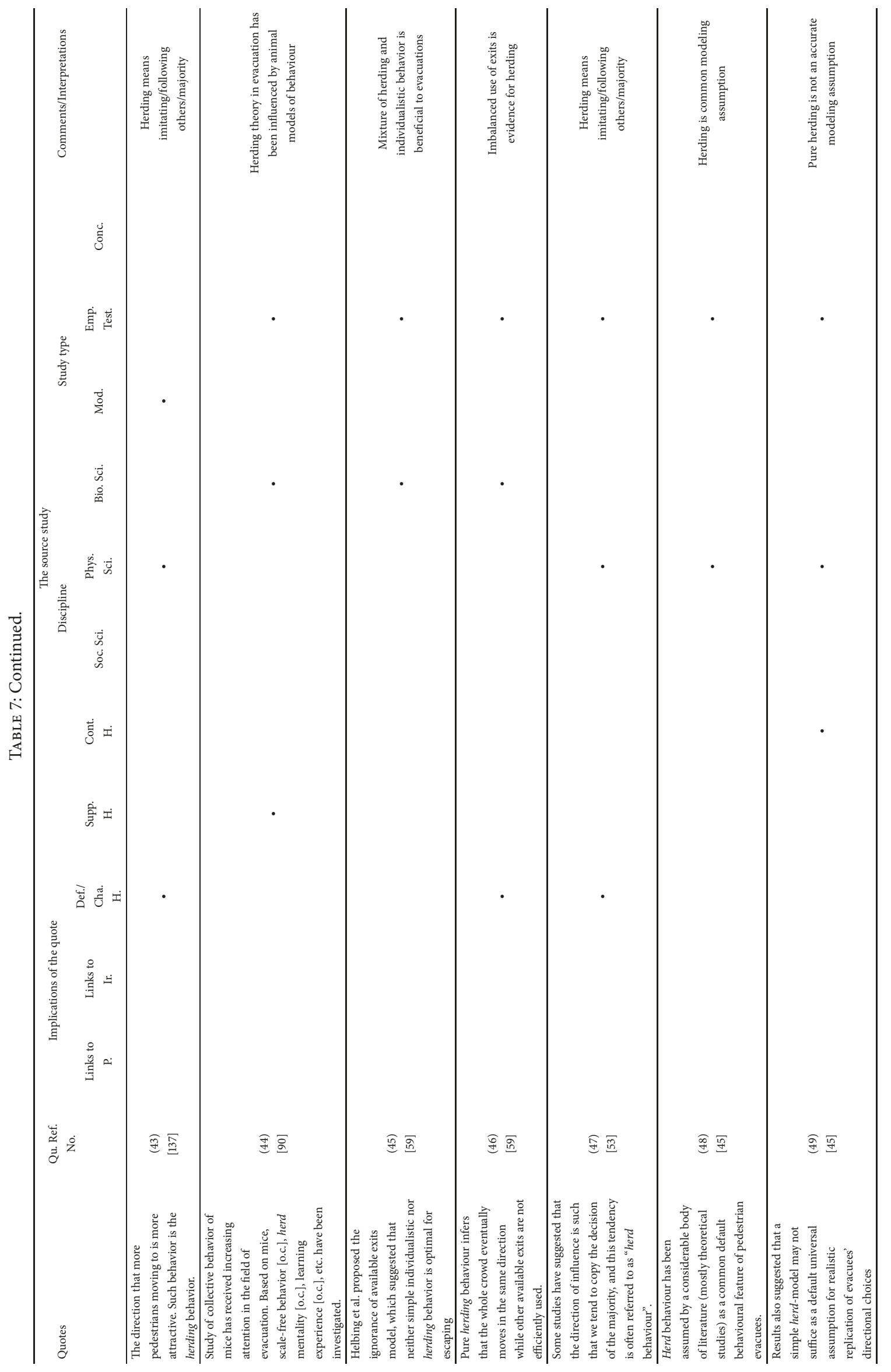




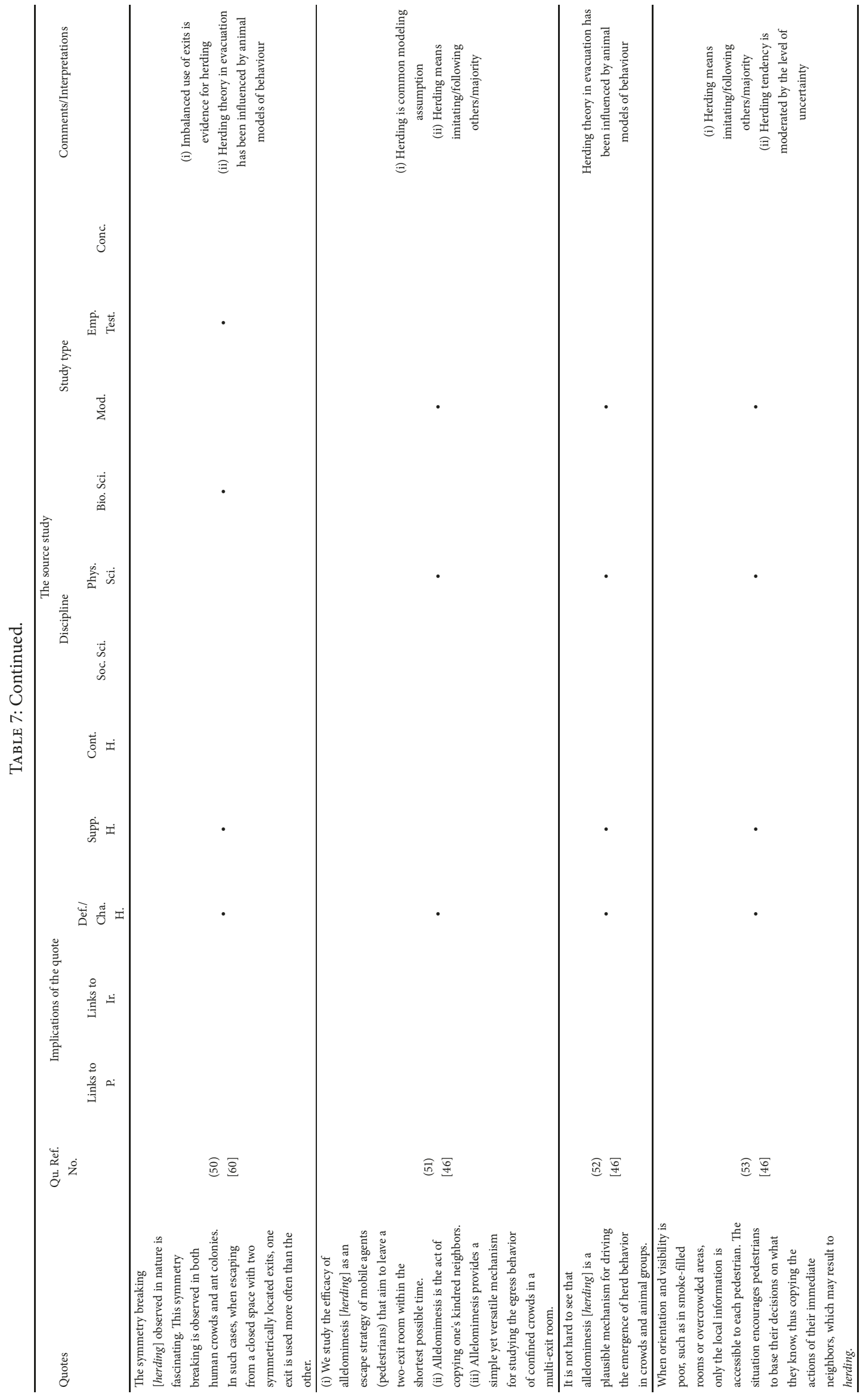




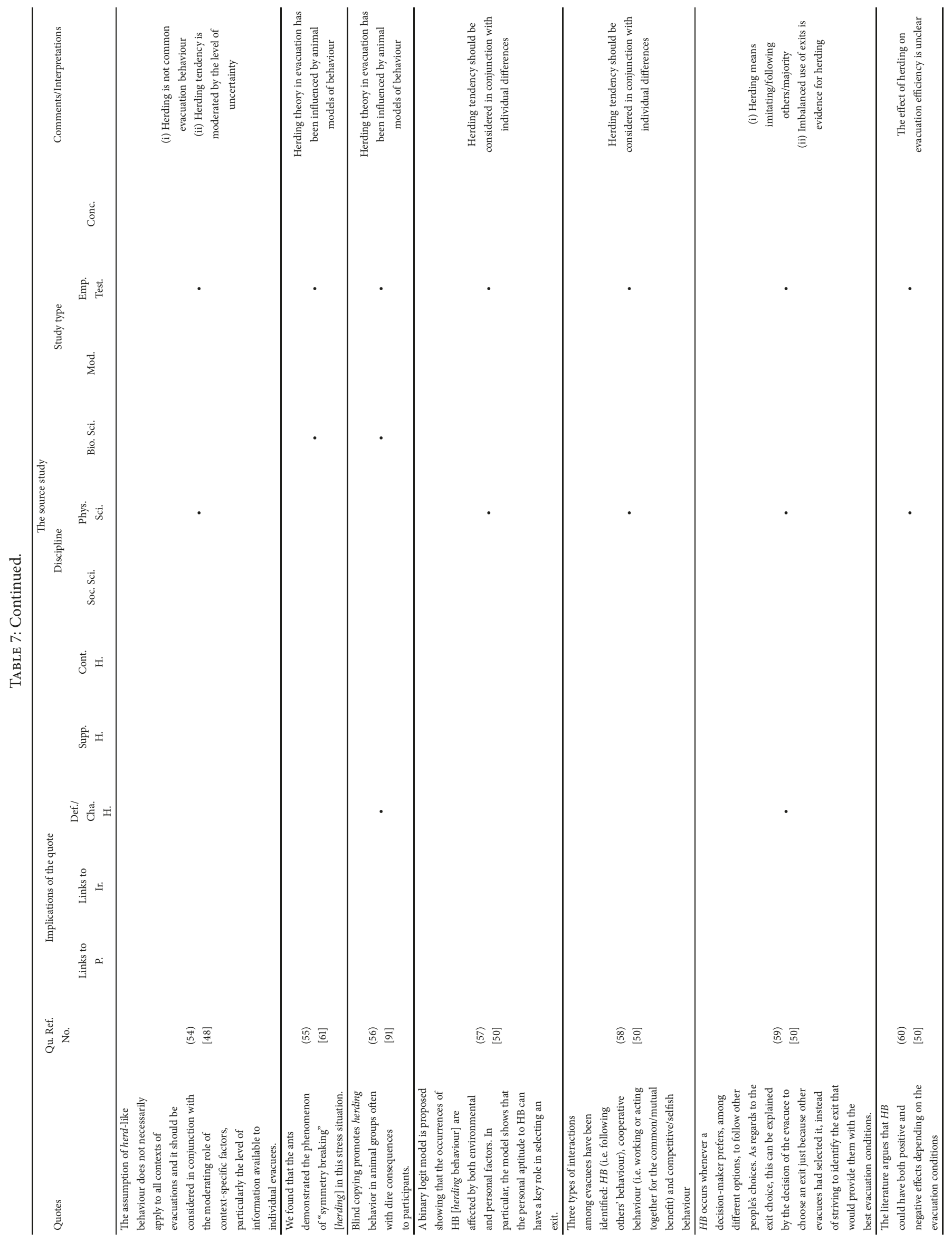




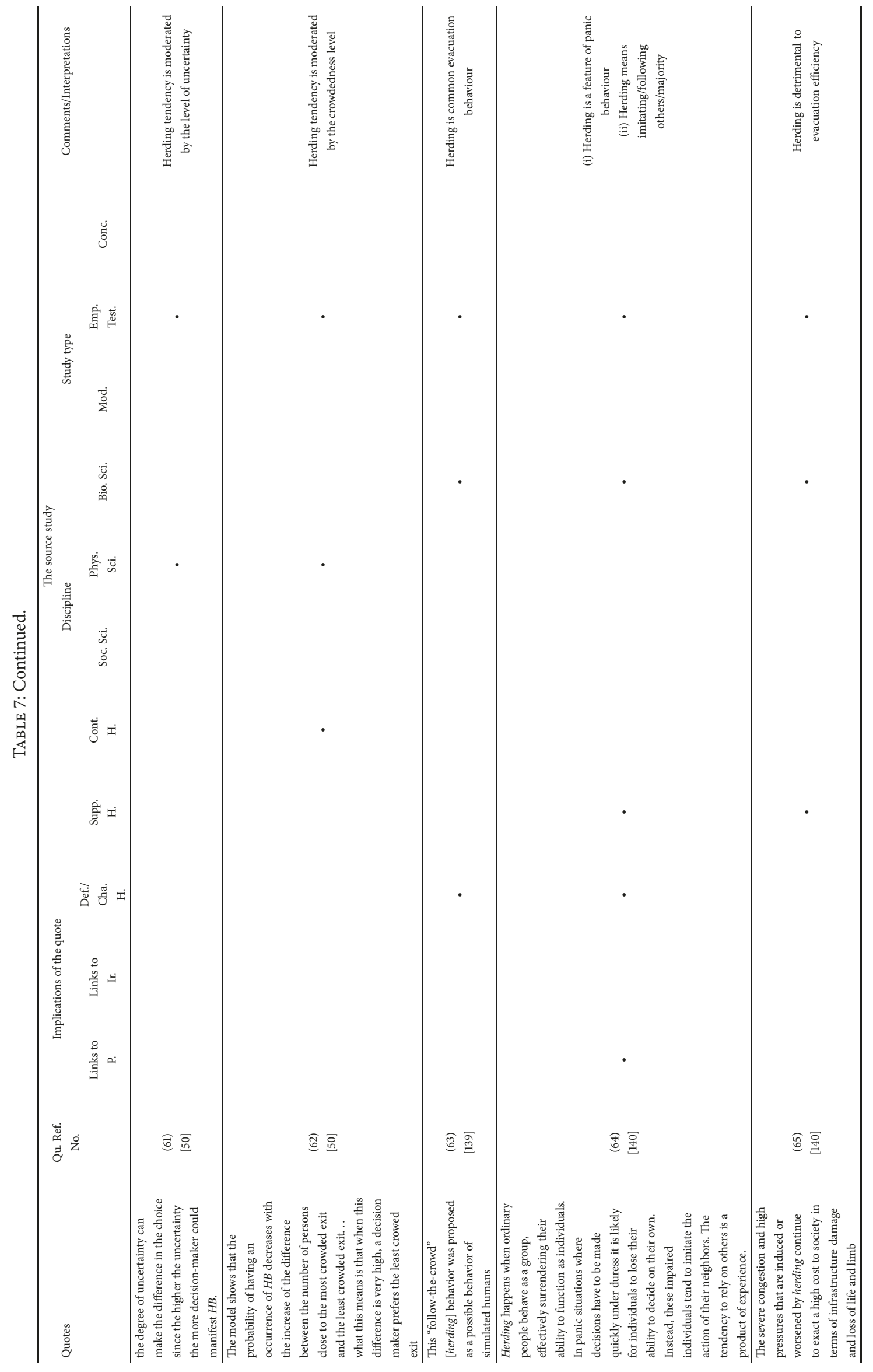




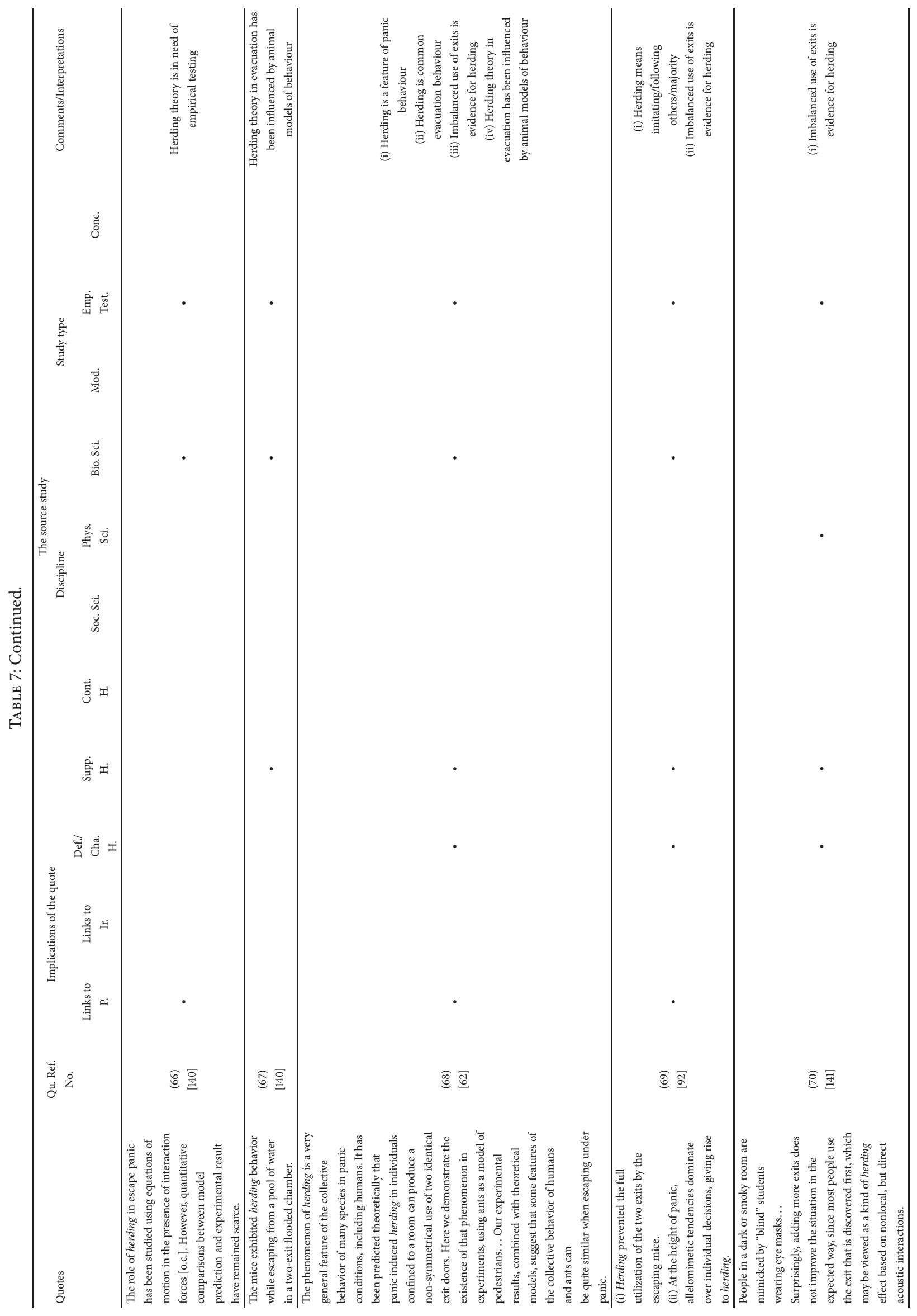




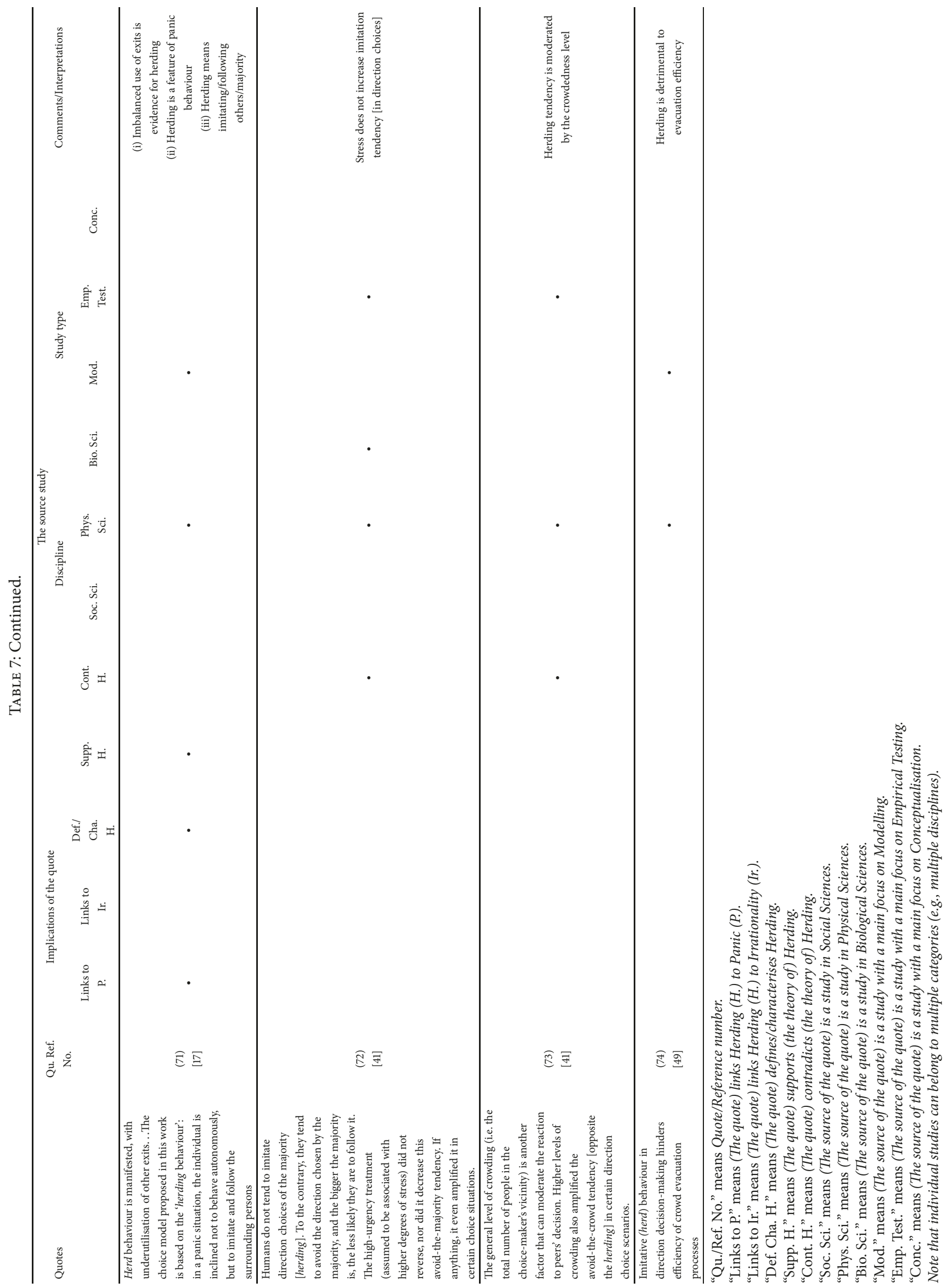




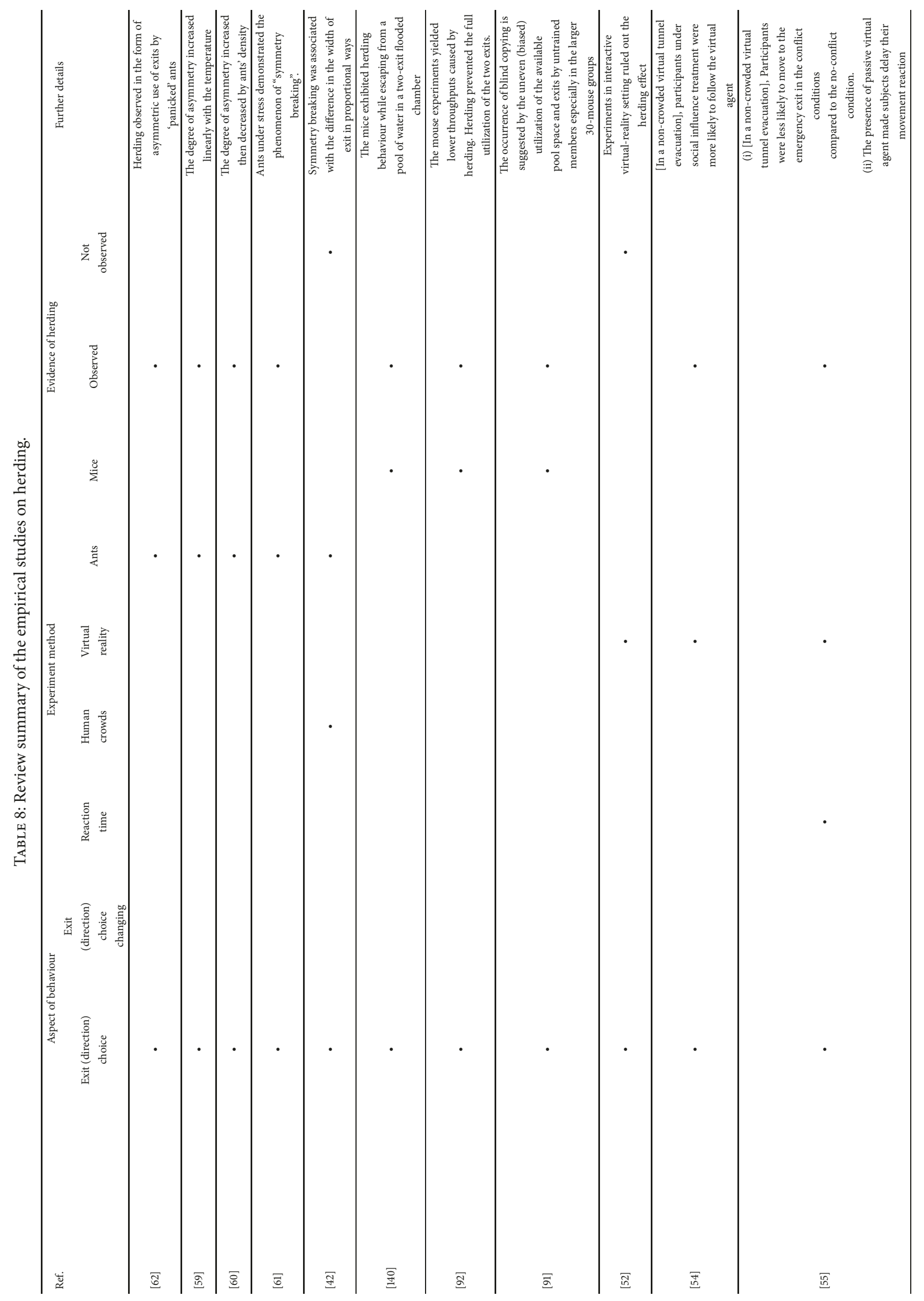


Journal of Advanced Transportation

53

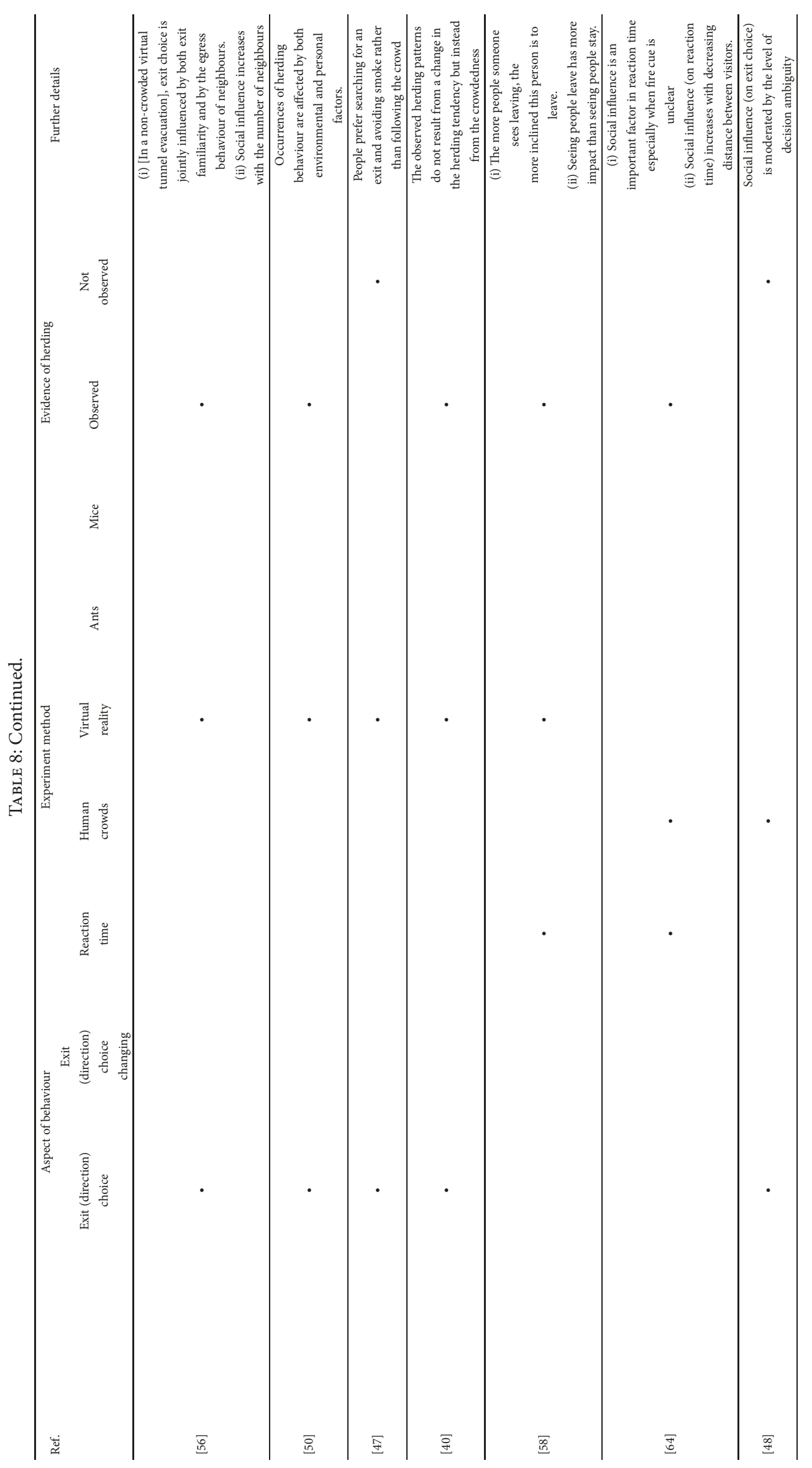




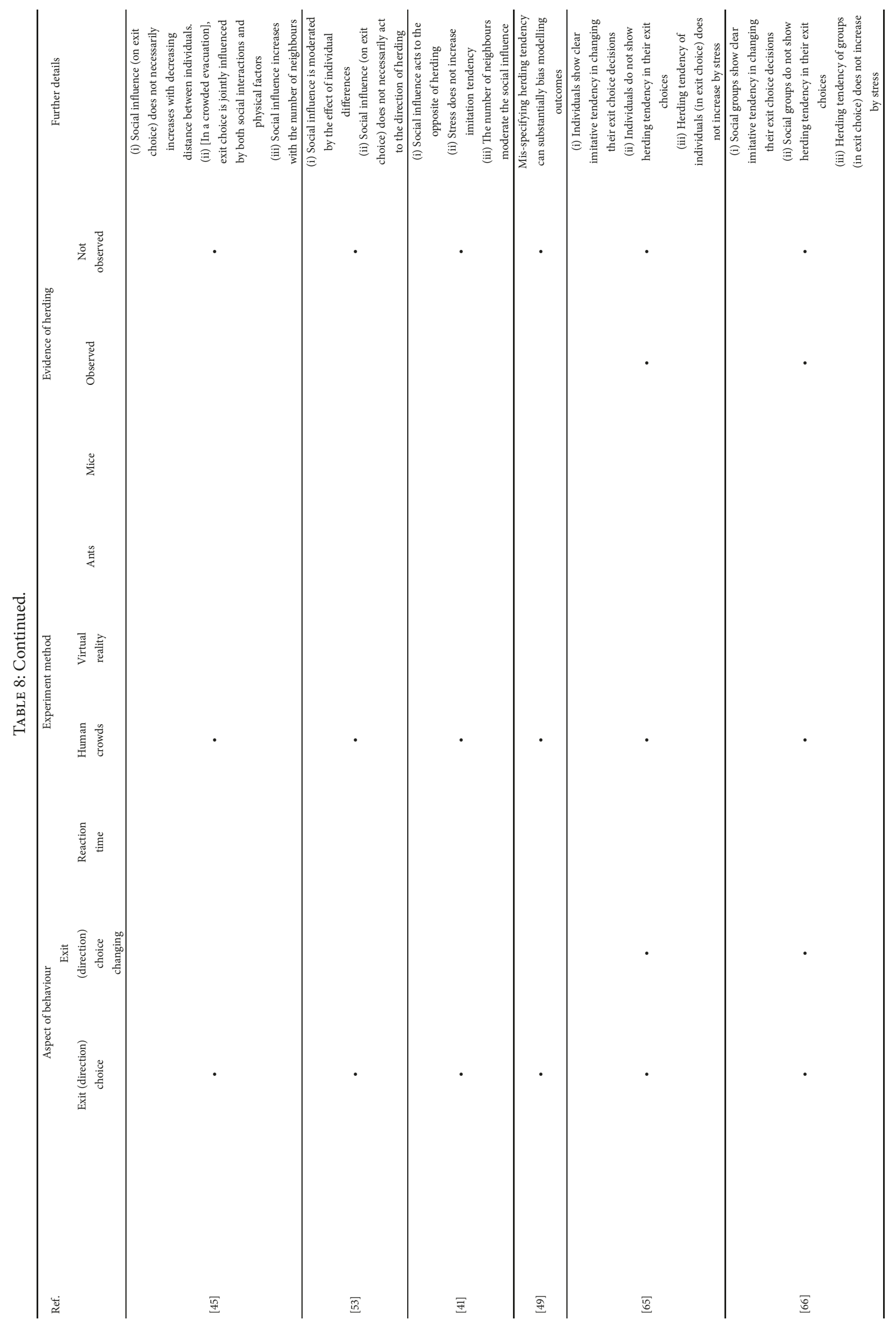




\section{References}

[1] X. Yang, Z. Wu, and Y. Li, "Difference between real-life escape panic and mimic exercises in simulated situation with implications to the statistical physics models of emergency evacuation: The 2008 Wenchuan earthquake," Physica A: Statistical Mechanics and its Applications, vol. 390, no. 12, pp. 2375-2380, 2011.

[2] C. Dias, M. Sarvi, O. Ejtemai, and M. Burd, "Elevated desired speed and change in desired direction: Effects on collective pedestrian flow characteristics," Transportation Research Record, vol. 2490, pp. 65-75, 2015.

[3] A. Seyfried, O. Passon, B. Steffen, M. Boltes, T. Rupprecht, and W. Klingsch, "New insights into pedestrian flow through bottlenecks," Transportation Science, vol. 43, no. 3, pp. 395-406, 2009.

[4] X. Li, T. Chen, L. Pan, S. Shen, and H. Yuan, "Lattice gas simulation and experiment study of evacuation dynamics," Physica A: Statistical Mechanics and its Applications, vol. 387, no. 22, pp. 5457-5465, 2008.

[5] E. L. Quarantelli, “The sociology of panic," 2001.

[6] M. Haghani and M. Sarvi, "Crowd behaviour and motion: Empirical methods," Transportation Research Part B: Methodological, vol. 107, pp. 253-294, 2018.

[7] M. Kobes, I. Helsloot, B. De Vries, and J. G. Post, "Building safety and human behaviour in fire: a literature review," Fire Safety Journal, vol. 45, no. 1, pp. 1-11, 2010.

[8] T. Wang, K. Huang, Y. Cheng, and X. Zheng, "Understanding herding based on a co-evolutionary model for strategy and game structure," Chaos, Solitons \& Fractals, vol. 75, pp. 84-90, 2015.

[9] J. Shen, X. Wang, and L. Jiang, "The influence of panic on the efficiency of escape," Physica A: Statistical Mechanics and its Applications, vol. 491, pp. 613-618, 2018.

[10] S. T. Rassia and C. I. Siettos, "Escape dynamics in office buildings: using molecular dynamics to quantify the impact of certain aspects of human behavior during emergency evacuation," Environmental Modeling \& Assessment, vol. 15, no. 5, pp. 411-418, 2010.

[11] F. Guo, X. Li, H. Kuang, Y. Bai, and H. Zhou, "An extended cost potential field cellular automata model considering behavior variation of pedestrian flow," Physica A: Statistical Mechanics and its Applications, vol. 462, pp. 630-640, 2016.

[12] D. Tong and D. Canter, "The decision to evacuate: a study of the motivations which contribute to evacuation in the event of fire," Fire Safety Journal, vol. 9, no. 3, pp. 257-265, 1985.

[13] J. Drury, "The role of social identity processes in mass emergency behaviour: An integrative review," European Review of Social Psychology, vol. 29, no. 1, pp. 38-81, 2018.

[14] N. Wagner and V. Agrawal, "An agent-based simulation system for concert venue crowd evacuation modeling in the presence of a fire disaster," Expert Systems with Applications, vol. 41, no. 6, pp. 2807-2815, 2014.

[15] J. D. Sime, "Affiliative behaviour during escape to building exits," Journal of Environmental Psychology, vol. 3, no. 1, pp. 21-41, 1983.

[16] Y. Song, J. Gong, Y. Li, T. Cui, L. Fang, and W. Cao, "Crowd evacuation simulation for bioterrorism in micro-spatial environments based on virtual geographic environments," Safety Science, vol. 53, pp. 105-113, 2013.

[17] M. Dell'Orco, M. Marinelli, and M. Ottomanelli, "Simulation of crowd dynamics in panic situations using a fuzzy logicbased behavioural model," Advances in Intelligent Systems and Computing, vol. 262, pp. 237-250, 2014.
[18] A. Kirchner and A. Schadschneider, "Simulation of evacuation processes using a bionics-inspired cellular automaton model for pedestrian dynamics," Physica A: Statistical Mechanics and its Applications, vol. 312, no. 1-2, pp. 260-276, 2002.

[19] D. Helbing, I. Farkas, and T. Vicsek, "Simulating dynamical features of escape panic," Nature, vol. 407, no. 6803, pp. 487490, 2000.

[20] M. Haghani, M. Sarvi, and Z. Shahhoseini, "When 'push' does not come to 'shove': Revisiting 'faster is slower' in collective egress of human crowds," Transportation Research Part A: Policy and Practice, vol. 122, pp. 51-69, 2019.

[21] Z. Shahhoseini, M. Sarvi, and M. Saberi, "Pedestrian crowd dynamics in merging sections: Revisiting the "faster-is-slower" phenomenon," Physica A: Statistical Mechanics and its Applications, vol. 491, pp. 101-111, 2018.

[22] X. Shi, Z. Ye, N. Shiwakoti, D. Tang, and J. Lin, "Examining effect of architectural adjustment on pedestrian crowd flow at bottleneck," Physica A: Statistical Mechanics and its Applications, vol. 522, pp. 350-364, 2019.

[23] S. Heliövaara, H. Ehtamo, D. Helbing, and T. Korhonen, "Patient and impatient pedestrians in a spatial game for egress congestion," Physical Review E: Statistical, Nonlinear, and Soft Matter Physics, vol. 87, no. 1, 2013.

[24] J. Drury, D. Novelli, and C. Stott, "Representing crowd behaviour in emergency planning guidance: 'mass panic' or collective resilience?" Resilience, vol. 1, no. 1, pp. 18-37, 2013.

[25] E. A. Heide, Common misconceptions about disasters: Panic, the, disaster syndrome, and looting, The first 72 hours: A community approach to disaster preparedness, 2004.

[26] G. Proulx, "A stress model for people facing a fire," Journal of Environmental Psychology, vol. 13, no. 2, pp. 137-147, 1993.

[27] J. Drury, C. Cocking, and S. Reicher, "Everyone for themselves? A comparative study of crowd solidarity among emergency survivors," British Journal of Social Psychology, vol. 48, no. 3, pp. 487-506, 2009.

[28] C. Cocking and J. Drury, “Talking about hillsborough: 'panic' as discourse in survivors' accounts of the 1989 football stadium disaster," Journal of Community \& Applied Social Psychology, vol. 24, no. 2, pp. 86-99, 2014.

[29] A. J. Glass, "Psychological aspects of disaster," Journal of the American Medical Association, vol. 171, no. 2, pp. 222-225, 1959.

[30] R. F. Fahy, G. Proulx, and L. Aiman, "Panic or not in fire: Clarifying the misconception," Fire and Materials, vol. 36, no. 5-6, pp. 328-338, 2012.

[31] J. Drury, D. Novelli, and C. Stott, "Psychological disaster myths in the perception and management of mass emergencies," Journal of Applied Social Psychology, vol. 43, no. 11, pp. 22592270, 2013.

[32] M. Haghani and M. Sarvi, "Rationality in collective escape behaviour: Identifying reference points of measurement at micro and macro levels," Journal of Advanced Transportation, 2019.

[33] H. H. Kelley, J. C. Condry, A. E. Dahlke, and A. H. Hill, "Collective behavior in a simulated panic situation," Journal of Experimental Social Psychology, vol. 1, no. 1, pp. 20-54, 1965.

[34] B. Sheppard, G. J. Rubin, J. K. Wardman, and S. Wessely, "Viewpoint: Terrorism and Dispelling the Myth of a Panic Prone Public," Journal of Public Health Policy, vol. 27, no. 3, pp. 219-245, 2006.

[35] L. Zhao, G. Yang, W. Wang et al., "Herd behavior in a complex adaptive system," Proceedings of the National Acadamy 
of Sciences of the United States of America, vol. 108, no. 37, pp. 15058-15063, 2011.

[36] X. Zheng and Y. Cheng, "Conflict game in evacuation process: a study combining cellular automata model," Physica A: Statistical Mechanics and its Applications, vol. 390, no. 6, pp. 1042-1050, 2011.

[37] X. Zheng and Y. Cheng, "Modeling cooperative and competitive behaviors in emergency evacuation: a game-theoretical approach," Computers \& Mathematics with Applications, vol. 62, no. 12, pp. 4627-4634, 2011.

[38] D. J. Low, "Statistical physics: Following the crowd," Nature, vol. 407, pp. 465-466, 2000.

[39] X. Pan, C. S. Han, K. Dauber, and K. H. Law, "A multiagent based framework for the simulation of human and social behaviors during emergency evacuations," AI \& Soc, vol. 22, pp. 113-132, 2007.

[40] M. Moussaïd, M. Kapadia, T. Thrash et al., "Crowd behaviour during high-stress evacuations in an immersive virtual environment," Journal of the Royal Society Interface, vol. 13, no. 122, p. 20160414, 2016.

[41] M. Haghani and M. Sarvi, "'Herding' in direction choicemaking during collective escape of crowds: How likely is it and what moderates it?" Safety Science, vol. 115, pp. 362-375, 2019.

[42] Q. Ji, C. Xin, S. Tang, and J. Huang, "Symmetry associated with symmetry break: Revisiting ants and humans escaping from multiple-exit rooms," Physica A: Statistical Mechanics and its Applications, vol. 492, pp. 941-947, 2018.

[43] L. Hong, J. Gao, and W. Zhu, "Self-evacuation modelling and simulation of passengers in metro stations," Safety Science, vol. 110, pp. 127-133, 2018.

[44] G. Albi, M. Bongini, E. Cristiani, and D. Kalise, "Invisible Control of Self-Organizing Agents Leaving Unknown Environments," SIAM Journal on Applied Mathematics, vol. 76, no. 4, pp. 1683-1710, 2016.

[45] M. Haghani and M. Sarvi, "Social dynamics in emergency evacuations: disentangling crowd's attraction and repulsion effects," Physica A: Statistical Mechanics and its Applications, vol. 475, pp. 24-34, 2017.

[46] G. J. Perez and C. Saloma, "Allelomimesis as escape strategy of pedestrians in two-exit confinements," Physica A: Statistical Mechanics and its Applications, vol. 388, no. 12, pp. 2469-2475, 2009.

[47] H. Li, L. Huang, Y. Zhang, and S. Ni, "Effects of intuition and deliberation on escape judgment and decision-making under different complexities of crisis situations," Safety Science, vol. 89, pp. 106-113, 2016.

[48] M. Haghani and M. Sarvi, "Following the crowd or avoiding it? Empirical investigation of imitative behaviour in emergency escape of human crowds," Animal Behaviour, vol. 124, pp. 47-56, 2017.

[49] M. Haghani and M. Sarvi, "Imitative (herd) behaviour in direction decision-making hinders efficiency of crowd evacuation processes," Safety Science, vol. 114, pp. 49-60, 2019.

[50] R. Lovreglio, A. Fonzone, L. dell'Olio, and D. Borri, "A study of herding behaviour in exit choice during emergencies based on random utility theory," Safety Science, vol. 82, pp. 421-431, 2016.

[51] M. Haghani and M. Sarvi, "Heterogeneity of decision strategy in collective escape of human crowds: On identifying the optimum composition," International Journal of Disaster Risk Reduction, vol. 35, 2019.
[52] N. W. Bode and E. A. Codling, "Human exit route choice in virtual crowd evacuations," Animal Behaviour, vol. 86, no. 2, pp. 347-358, 2013.

[53] M. Haghani and M. Sarvi, "How perception of peer behaviour influences escape decision making: The role of individual differences," Journal of Environmental Psychology, vol. 51, pp. 141-157, 2017.

[54] M. Kinateder, E. Ronchi, D. Gromer et al., "Social influence on route choice in a virtual reality tunnel fire," Transportation Research Part F: Traffic Psychology and Behaviour, vol. 26, pp. 116-125, 2014.

[55] M. Kinateder, M. Müller, M. Jost, A. Mühlberger, and P. Pauli, "Social influence in a virtual tunnel fire - Influence of conflicting information on evacuation behavior," Applied Ergonomics, vol. 45, no. 6, pp. 1649-1659, 2014.

[56] M. Kinateder, B. Comunale, and W. H. Warren, "Exit choice in an emergency evacuation scenario is influenced by exit familiarity and neighbor behavior," Safety Science, vol. 106, pp. 170-175, 2018.

[57] T. Wang, D. Wang, and F. Wang, "Quantifying herding effects in crowd wisdom," in Proceedings of the 20th ACM SIGKDD international conference on Knowledge discovery and data mining, pp. 1087-1096, ACM, 2014.

[58] M. Van den Berg, R. van Nes, and S. Hoogendoorn, "Estimating choice models to quantify the effect of herding on the decision to evacuate: Application of a serious gaming experimental setup," Transportation Research Record, vol. 2672, no. 1, pp. 161$170,2018$.

[59] Y. Chung, C. Lin, and E. Ito, "Heat-induced symmetry breaking in ant (Hymenoptera: Formicidae) escape behavior," PLOS ONE, vol. 12, no. 3, p. e0173642, 2017.

[60] G. Li, D. Huan, B. Roehner et al., "Symmetry Breaking on Density in Escaping Ants: Experiment and Alarm Pheromone Model," PLoS ONE, vol. 9, no. 12, p. el14517, 2014.

[61] S. Wang, S. Cao, Q. Wang, L. Lian, and W. Song, "Effect of exit locations on ants escaping a two-exit room stressed with repellent," Physica A: Statistical Mechanics and its Applications, vol. 457, pp. 239-254, 2016.

[62] E. Altshuler, O. Ramos, Y. Núñez, J. Fernández, A. J. BatistaLeyva, and C. Noda, "Symmetry breaking in escaping ants," The American Naturalist, vol. 166, no. 6, pp. 643-649, 2005.

[63] M. Mayor, Longman dictionary of contemporary English, Pearson Education, India, 2009.

[64] D. Nilsson and A. Johansson, "Social influence during the initial phase of a fire evacuation-Analysis of evacuation experiments in a cinema theatre," Fire Safety Journal, vol. 44, no. 1, pp. 71-79, 2009.

[65] M. Haghani, M. Sarvi, and Z. Shahhoseini, Experimenting evacuation decision-making under high and low levels of urgency: Disaggregate data and models of reaction time, exit choice and exit-choice adaptation Under review, 2019.

[66] M. Haghani, M. Sarvi, Z. Shahhoseini, and M. Boltes, "Dynamics of social groups' decision-making in evacuations," Transportation Research Part C: Emerging Technologies, vol. 104, pp. 135-157, 2019.

[67] M. Haghani, Humans' decision-making during emergency evacuations of crowded environments: behavioural analyses and econometric modelling perspectives, 2017.

[68] N. W. Bode and E. A. Codling, "Exploring determinants of premovement delays in a virtual crowd evacuation experiment," Fire Technology, 2018. 
[69] E. R. Galea, S. J. Deere, C. G. Hopkin, and H. Xie, "Evacuation response behaviour of occupants in a large theatre during a live performance," Fire and Materials, vol. 41, no. 5, pp. 467-492, 2017.

[70] M. Haghani, M. Sarvi, and L. Scanlon, "Simulating preevacuation times using hazard-based duration models: Is waiting strategy more efficient than instant response?" Safety Science, vol. 117, pp. 339-351, 2019.

[71] Z. Fang, Q. Li, Q. Li, L. D. Han, and D. Wang, "A proposed pedestrian waiting-time model for improving space-time use efficiency in stadium evacuation scenarios," Building and Environment, vol. 46, no. 9, pp. 1774-1784, 2011.

[72] M. Kinateder, P. Pauli, M. Müller et al., "Human behaviour in severe tunnel accidents: Effects of information and behavioural training," Transportation Research Part F: Traffic Psychology and Behaviour, vol. 17, pp. 20-32, 2013.

[73] E. Ronchi, M. Kinateder, M. Müller et al., "Evacuation travel paths in virtual reality experiments for tunnel safety analysis," Fire Safety Journal, vol. 71, pp. 257-267, 2015.

[74] M. Haghani and M. Sarvi, "Pedestrian crowd tactical-level decision making during emergency evacuations," Journal of Advanced Transportation, vol. 50, no. 8, pp. 1870-1895, 2016.

[75] N. W. Bode, A. U. Kemloh Wagoum, and E. A. Codling, "Human responses to multiple sources of directional information in virtual crowd evacuations," Journal of the Royal Society Interface, vol. 11, no. 91, p. 20130904, 2014.

[76] M. Haghani and M. Sarvi, "Identifying Latent Classes of Pedestrian Crowd Evacuees, Transportation Research Record," Transportation Research Record, vol. 2560, pp. 67-74, 2016.

[77] M. Haghani and M. Sarvi, "Stated and revealed exit choices of pedestrian crowd evacuees," Transportation Research Part B: Methodological, vol. 95, pp. 238-259, 2017.

[78] E. R. Gwynne, P. J. Galea, and S. Lawrence, "Adaptive decisionmaking in response to crowd formations in building EXODUS," Journal of Applied Fire Science, vol. 8, no. 4, pp. 301-325, 1999.

[79] S. Gwynne, The introduction of adaptive social decision-making in the mathematical modelling of egress behaviour, University of Greenwich, 2000.

[80] W. Liao, A. U. Kemloh Wagoum, and N. W. Bode, "Route choice in pedestrians: determinants for initial choices and revising decisions," Journal of the Royal Society Interface, vol. 14, no. 127, p. 20160684, 2017.

[81] S. Gwynne and A. Hunt, "Why model evacuee decisionmaking?” Safety Science, vol. 110, pp. 457-466, 2018.

[82] N. W. F. Bode, A. U. Kemloh Wagoum, and E. A. Codling, "Information use by humans during dynamic route choice in virtual crowd evacuations," Royal Society Open Science, vol. 2, no. 1, 2015.

[83] M. Haghani and M. Sarvi, "Human exit choice in crowded built environments: Investigating underlying behavioural differences between normal egress and emergency evacuations," Fire Safety Journal, vol. 85, pp. 1-9, 2016.

[84] M. Haghani, M. Sarvi, and Z. Shahhoseini, "Accommodating taste heterogeneity and desired substitution pattern in exit choices of pedestrian crowd evacuees using a mixed nested logit model," Journal of Choice Modelling, vol. 16, pp. 58-68, 2015.

[85] D. Duives and H. Mahmassani, "Exit choice decisions during pedestrian evacuations of buildings," Transportation Research Record, no. 2316, pp. 84-94, 2012.
[86] M. Haghani and M. Sarvi, "Simulating dynamics of adaptive exit-choice changing in crowd evacuations: Model implementation and behavioural interpretations," Transportation Research Part C: Emerging Technologies, vol. 103, pp. 56-82, 2019.

[87] D. R. Parisi, S. A. Soria, and R. Josens, "Faster-is-slower effect in escaping ants revisited: Ants do not behave like humans," Safety Science, vol. 72, pp. 274-282, 2015.

[88] M. Borenstein, L. V. Hedges, J. P. Higgins, and H. R. Rothstein, Introductionto Meta-Analysis, JohnWiley\&SonsLtd, WestSussex, UK, 2009.

[89] J. Adrian, N. Bode, M. Amos et al., "A glossary for research on human crowd dynamics," Collective Dynamics, vol. 4, p. 13, 2019.

[90] T. Zhang, X. Zhang, S. Huang, C. Li, and S. Lu, "Collective behavior of mice passing through an exit under panic," Physica A: Statistical Mechanics and its Applications, vol. 496, pp. 233242, 2018.

[91] C. Saloma, G. J. Perez, C. A. Gavile, J. J. Ick-Joson, C. PalmesSaloma, and A. Sánchez, "Prior individual training and selforganized queuing during group emergency escape of mice from water pool," PLoS ONE, vol. 10, no. 2, p. e0118508, 2015.

[92] C. Saloma, G. J. Perez, G. Tapang, M. Lim, and C. PalmesSaloma, "Self-organized queuing and scale-free behavior in real escape panic," Proceedings of the National Acadamy of Sciences of the United States of America, vol. 100, no. 21, pp. 11947-11952, 2003.

[93] P. Lin, J. Ma, T. Liu, T. Ran, Y. Si, and T. Li, "An experimental study of the "faster-is-slower" effect using mice under panic," Physica A: Statistical Mechanics and its Applications, vol. 452, pp. 157-166, 2016.

[94] P. Lin, J. Ma, T. Y. Liu et al., "An experimental study of the impact of an obstacle on the escape efficiency by using mice under high competition," Physica A: Statistical Mechanics and its Applications, vol. 482, pp. 228-242, 2017.

[95] F.-Y. Wu, G.-Y. Wang, Y.-L. Si, and P. Lin, "An experimental study on the exit location on the evacuation efficiency under high competition condition," Procedia Engineering, vol. 211, pp. 801-809, 2018.

[96] A. Garcimartín, J. M. Pastor, L. M. Ferrer, J. J. Ramos, C. MartínGómez, and I. Zuriguel, "Flow and clogging of a sheep herd passing through a bottleneck," Physical Review E: Statistical, Nonlinear, and Soft Matter Physics, vol. 91, no. 2, 2015.

[97] I. Zuriguel, J. Olivares, J. M. Pastor et al., "Effect of obstacle position in the flow of sheep through a narrow door," Physical Review E: Statistical, Nonlinear, and Soft Matter Physics, vol. 94, no. 3, 2016.

[98] H. Li, Y. Shi, Y. Zhang, L. Huang, and A. A. Gao, "Influence of information sources on escape judgment with intuition and after deliberation," Safety Science, vol. 78, pp. 101-110, 2015.

[99] N. Bellomo, D. Clarke, L. Gibelli, P. Townsend, and B. Vreugdenhil, "Human behaviours in evacuation crowd dynamics: From modelling to "big data" toward crisis management," Physics of Life Reviews, vol. 18, pp. 1-21, 2016.

[100] N. Shiwakoti and M. Sarvi, "Enhancing the panic escape of crowd through architectural design," Transportation Research Part C: Emerging Technologies, vol. 37, pp. 260-267, 2013.

[101] J. Wang, L. Zhang, Q. Shi, P. Yang, and X. Hu, "Modeling and simulating for congestion pedestrian evacuation with panic," Physica A: Statistical Mechanics and its Applications, vol. 428, pp. 396-409, 2015.

[102] D. Helbing and A. Johansson, Pedestrian, crowd and evacuation dynamics, Encyclopedia of Complexity and Systems Science, vol. $16,2010$. 
[103] D. Helbing, I. J. Farkas, and T. Vicsek, "Simulation of pedestrian crowds in normal and evacuation situations," in Pedestrian and Evacuation Dynamics, M. Schreckenberg and S. D. Sharma, Eds., pp. 21-58, Springer, Berlin, Germany, 2002.

[104] D. Zhao, L. Yang, and J. Li, “Occupants' behavior of going with the crowd based on cellular automata occupant evacuation model," Physica A: Statistical Mechanics and its Applications, vol. 387, no. 14, pp. 3708-3718, 2008.

[105] J. Wang, M. Chen, W. Yan, Y. Zhi, and Z. Wang, "A utility threshold model of herding-panic behavior in evacuation under emergencies based on complex network theory," Simulation, vol. 93, no. 2, pp. 123-133, 2016.

[106] T. Elzie, E. Frydenlund, A. J. Collins, and R. M. Robinson, "Panic that spreads sociobehavioral contagion in pedestrian evacuations," Transportation Research Record, vol. 2586, pp. 18, 2016.

[107] L. Fu, W. Song, W. Lv, and S. Lo, "Simulation of emotional contagion using modified SIR model: a cellular automaton approach," Physica A: Statistical Mechanics and Its Applications, vol. 405, pp. 380-391, 2014.

[108] D. R. PARISI and C. O. DORSO, "The role of panic in the room evacuation process," International Journal of Modern Physics C, vol. 17, no. 03, pp. 419-434, 2011.

[109] D. R. Parisi and C. O. Dorso, "Microscopic dynamics of pedestrian evacuation," Physica A: Statistical Mechanics and its Applications, vol. 354, no. 1-4, pp. 606-618, 2005.

[110] G. Kouskoulis and C. Antoniou, "Systematic review of pedestrian simulation models with a focus on emergency situations," Transportation Research Record, vol. 2604, no. 1, pp. 111-119, 2017.

[111] D. Helbing and P. Mukerji, "Crowd disasters as systemic failures: analysis of the Love Parade disaster," EPJ Data Science, vol. 1, no. 1, 2012.

[112] B. Durodié and S. Wessely, "Resilience or panic? The public and terrorist attack," The Lancet, vol. 360, no. 9349, pp. 1901-1902, 2002.

[113] J. Drury, C. Cocking, S. Reicher et al., "Cooperation versus competition in a mass emergency evacuation: A new laboratory simulation and a new theoretical model," Behavior Research Methods, vol. 41, no. 3, pp. 957-970, 2009.

[114] L. Clarke, Panic: myth or reality?, contexts, 2002.

[115] N. R. Johnson, "Panic at "The Who Concert Stampede": An Empirical Assessment," Social Problems, vol. 34, no. 4, pp. 362373, 1987.

[116] J. Drury, C. Cocking, and S. Reicher, "The nature of collective resilience: Survivor reactions to the 2005 London bombings," International Journal of Mass Emergencies and Disasters, vol. 27, pp. 66-95, 2009.

[117] B. E. Aguirre, "Emergency evacuations, panic, and social psychology," Psychiatry, vol. 68, no. 2, pp. 121-129, 2005.

[118] M. Moussaïd and M. Trauernicht, "Patterns of cooperation during collective emergencies in the help-or-escape social dilemma," Scientific Reports, vol. 6, no. 1, 2016.

[119] G. Dezecache, "Human collective reactions to threat," Wiley Interdisciplinary Reviews: Cognitive Science, vol. 6, no. 3, pp. 209-219, 2015.

[120] N. R. Johnson, "Panic and the Breakdown of Social Order: Popular Myth, Social Theory, Empirical Evidence," Sociological Focus, vol. 20, no. 3, pp. 171-183, 1987.

[121] I. von Sivers, A. Templeton, F. Künzner et al., "Modelling social identification and helping in evacuation simulation," Safety Science, vol. 89, pp. 288-300, 2016.
[122] N. T. Ouellette, "Flowing crowds," Science, vol. 363, no. 6422, pp. 27-28, 2019.

[123] A. R. Mawson, "Understanding Mass Panic and Other Collective Responses to Threat and Disaster," Psychiatry: Interpersonal and Biological Processes, vol. 68, no. 2, pp. 95-113, 2005.

[124] A. Grimm, L. Hulse, M. Preiss, and S. Schmidt, "Behavioural, emotional, and cognitive responses in European disasters: results of survivor interviews," Disasters, vol. 38, no. 1, pp. 62-83, 2014.

[125] J. Drury and C. Cocking, “The mass psychology of disasters and emergency evacuations: A research report and implications for practice," Citeseer, 2007.

[126] G. Proulx, Understanding Human Behaviour in Stressful Situations, 2002.

[127] A. E. Norwood, "Debunking the Myth of Panic," Psychiatry: Interpersonal and Biological Processes, vol. 68, no. 2, pp. 114-114, 2005.

[128] E. L. Quarantelli, "Conventional beliefs and counterintuitive realities," Social Research, vol. 75, no. 3, pp. 873-904, 2008.

[129] D. P. Schultz, "Theories of Panic Behavior: A Review," The Journal of Social Psychology, vol. 66, no. 1, pp. 31-40, 1965.

[130] D. Helbing, A. Johansson, and H. Z. Al-Abideen, "Dynamics of crowd disasters: an empirical study," Physical Review E, Stat Nonlinear Soft Matter Phys, 2007.

[131] C. Rogsch, M. Schreckenberg, E. Tribble, W. Klingsch, and T. Kretz, "An overview about mass-emergencies and their origins all over the world for recent years," in Pedestrian and Evacuation Dynamics 2008, pp. 743-755, Springer, 2010.

[132] J. Drury and C. Stott, "Contextualising the crowd in contemporary social science," Contemporary Social Science, vol. 6, no. 3, pp. 275-288, 2011.

[133] D. Schweingruber and R. T. Wohlstein, "The madding crowd goes to school: myths about crowds in introductory sociology textbooks," Teaching Sociology, vol. 33, no. 2, pp. 136-153, 2016.

[134] N. Wijermans, Understanding crowd behaviour, [PhD. thesis], University of Groningen, Groningen, 2011.

[135] J. M. Chertkoff, R. H. Kushigian, and M. Mccool Jr, "Interdependent exiting: The effects of group size, time limit, and gender on the coordination of exiting," Journal of Environmental Psychology, vol. 16, no. 2, pp. 109-121, 1996.

[136] I. Von Sivers, A. Templeton, G. Köster, J. Drury, and A. Philippides, "Humans do not always act selfishly: Social identity and helping in emergency evacuation simulation," Transportation Research Procedia, vol. 2, pp. 585-593, 2014.

[137] Y. Zheng, X. Li, N. Zhu, B. Jia, and R. Jiang, "Evacuation dynamics with smoking diffusion in three dimension based on an extended Floor-Field model," Physica A: Statistical Mechanics and its Applications, vol. 507, pp. 414-426, 2018.

[138] M. L. Chu, P. Parigi, K. Law, and J. Latombe, "Modeling social behaviors in an evacuation simulator," Computer Animation and Virtual Worlds, vol. 25, no. 3-4, pp. 373-382, 2014.

[139] S. Boari, R. Josens, D. R. Parisi, and J. A. Marshall, "Efficient egress of escaping ants stressed with temperature," PLOS ONE, vol. 8, no. 11, p. e81082, 2013.

[140] N. Waldau, P. Gattermann, H. Knoflacher, and M. Schreckenberg, Pedestrian and Evacuation Dynamics 2005, Springer, Berlin, Heidelberg, Germany, 2007.

[141] M. Isobe, D. Helbing, and T. Nagatani, "Experiment, theory, and simulation of the evacuation of a room without visibility," Physical Review E: Statistical, Nonlinear, and Soft Matter Physics, vol. 69 , no. $6,2004$. 


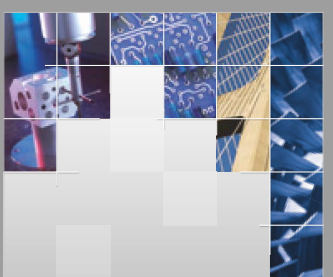

\section{Enfincering}
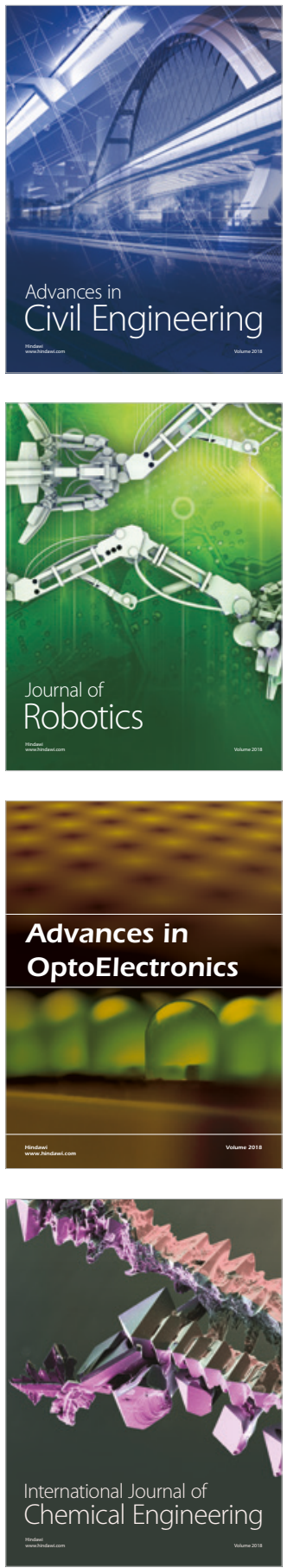

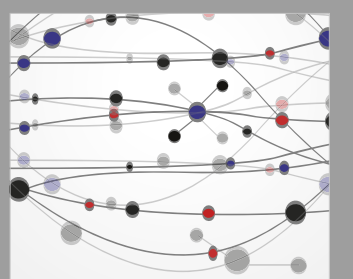

\section{Rotating \\ Machinery}

The Scientific World Journal

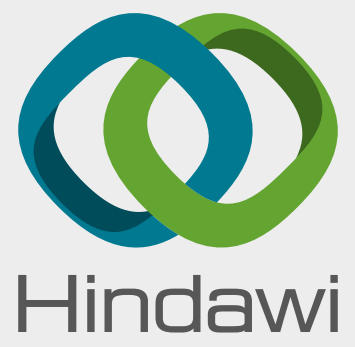

Submit your manuscripts at

www.hindawi.com
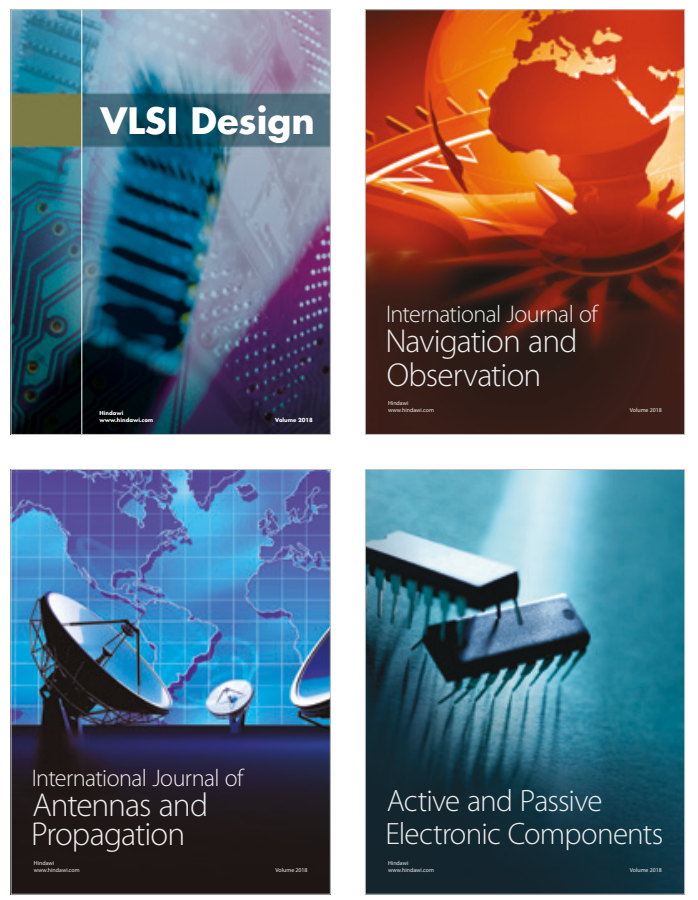
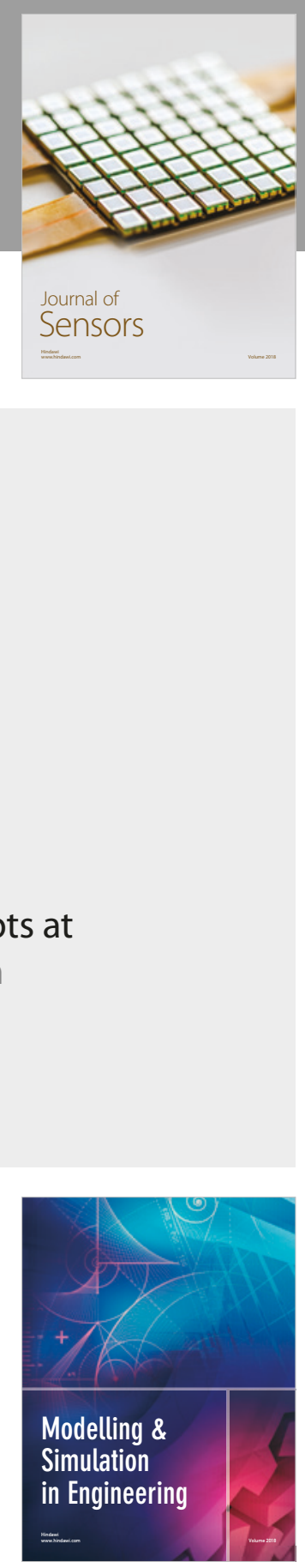

\section{Advances \\ Multimedia}
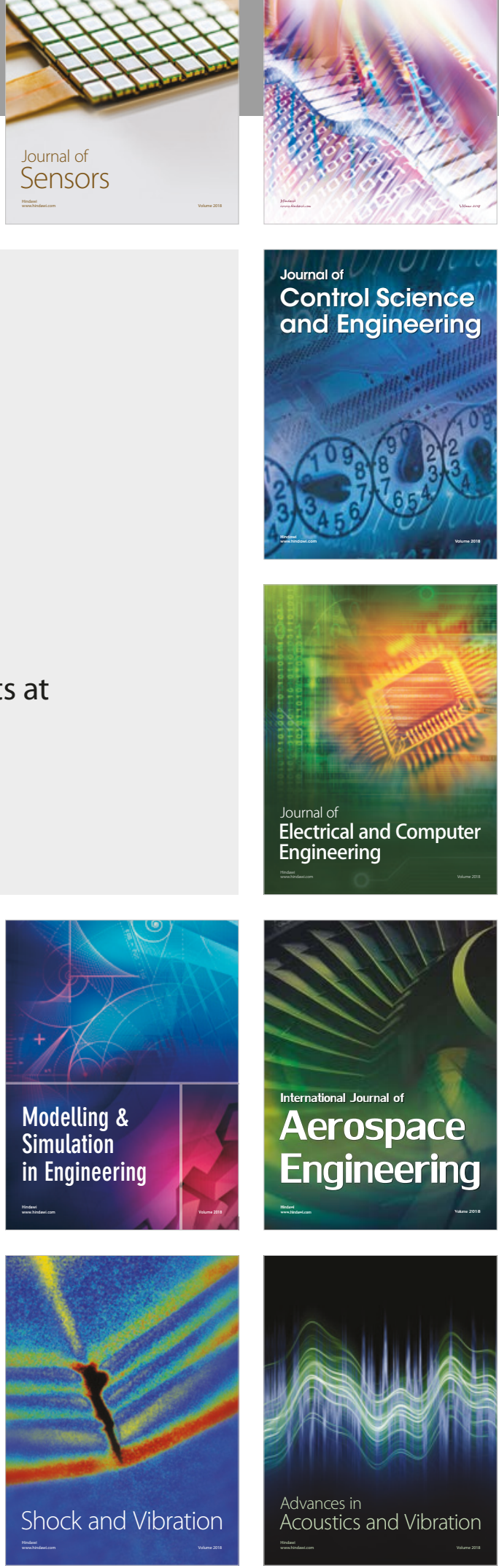